St er eosel ect i ve synt heses of (E) - $\alpha, \beta$

- di dehydroami no aci d and pept i de cont ai ni ng its resi due utilizing oxazol i di none deri vative

\begin{tabular}{|l|l|}
\hline 著者 & $\begin{array}{l}\text { Konet ani M ki, I har a Kohei, Ki mur a Rum , } \\
\text { Ki noshi t a H deki }\end{array}$ \\
\hline $\begin{array}{l}\text { j our nal or } \\
\text { publ i cat i on ti t e }\end{array}$ & Bul I et i $\mathrm{n}$ of the Chem cal Soci et y of Japan \\
\hline vol une & 82 \\
\hline number & 3 \\
\hline page range & 364 -380 \\
\hline year & $2009-01-01$ \\
\hline URL & ht t p: //hdl . handl e. net /2297/19682 \\
\hline
\end{tabular}




\title{
Stereoselective Syntheses of $(E)-\alpha, \beta$-Didehydroamino Acid and Peptide Containing Its Residue Utilizing Oxazolidinone Derivative
}

\author{
Miki Kometani, Kohki Ihara, Rumi Kimura, and Hideki Kinoshita* \\ Division of Material Sciences, Graduate School of Natural Science and Technology, Kanazawa University, \\ Kakuma, Kanazawa 920-1192
}

Received April 17, 2008; E-mail: khideki@p2223.nsk.ne.jp

Reaction of methyl $N$-Boc- $N$-phenoxycarbonylglycinate with various aldehydes afforded the corresponding cis-4,5oxazolidinone derivatives, which were effectively converted to $(E)-\alpha, \beta$-didehydroamino acids by means of a base. Furthermore, N-deprotection of the oxazolidinone derivatives and subsequent coupling reaction with Boc-amino acid furnished the corresponding dipeptides, which were transformed to dipeptide containing $\alpha, \beta$-didehydroamino acid with high E selectivity.

Much attention has been focused on naturally occurring peptides containing $\alpha, \beta$-didehydroamino acids ${ }^{1}$ and their derivatives from the view point of both synthetic interest and the relationship between their structure and bioactivities. ${ }^{2}$

Therefore, much work related to the preparation of $\alpha, \beta$ didehydroamino acids has been reported in which the products bearing thermodynamically controlled alkene geometry, $(Z)$ $\alpha, \beta$-didehydroamino acids were predominantly formed. ${ }^{3}$ In previous papers, we have reported that $N$-t-butoxycarbonyl (Boc)- or $N$-benzyloxycarbonyl ( $Z$ )- $\alpha$-tosylglycine ester was reacted with either a variety of nitro alkanes in the presence of base or various aldehydes in the presence of base and tributylphosphine to afford the corresponding $\alpha, \beta$-didehydroamino acid derivatives with high $\mathrm{Z}$ selectivity in good yields. ${ }^{4}$ The latter procedure was successfully applied to the synthesis of optically active 4-hydroxyprolines. ${ }^{5}$

On the contrary, there are few reports ${ }^{6}$ concerning stereoselective synthesis of $(E)$ - $\alpha, \beta$-didehydroamino acid since its stereochemistry is not always thermodynamically favored, for example, 1) stereoselective elimination of hydrogen chloride from erythro- $\beta$-chloro- $\alpha$-amino acid derived from threo- $\beta$ hydroxy- $\alpha$-amino acid derivative, ${ }^{6 a} 2$ ) stereospecific dehydration of erythro- $\beta$-hydroxy- $\alpha$-amino acid derivative using DAST [(diethylamino)sulfur trifluoride], ${ }^{6 \mathrm{~b}} 3$ ) formation of cyclic cis-sulfamidite starting from erythro- $\beta$-hydroxy- $\alpha$ amino acid derivative and thionyl chloride, and subsequent elimination of sulfur dioxide, ${ }^{6 c}$ 4) Suzuki coupling of $\beta$ bromo- $\alpha, \beta$-didehydroamino acid derivative with monosubstituted boronic acid, ${ }^{6 \mathrm{~d}} 5$ ) stereospecific dehydration of erythro$\beta$-hydroxy- $\alpha$-amino acid derivative using Martin's sulfurane [diphenylbis(1,1,1,3,3,3-hexafluoro-2-phenyl-2-propyl)sulfurane], $\left.{ }^{6 e} 6\right)$ stereospecific dehydration of threo- $\beta$-hydroxy$\alpha$-amino acid derivative with EDC [1-ethyl-3-(3-dimethylaminopropyl)carbodiimide], ${ }^{6 f} 7$ ) oxidation of 3-S-benzylthioamino acid derivative to the sulfoxide, followed by thermolysis to form $\alpha, \beta$-didehydroamino acid. ${ }^{3 \mathrm{~b}}$

Although enantiomerically pure $\beta$-hydroxy- $\alpha$-amino acid derivative or $\beta$-bromo- $\alpha, \beta$-didehydroamino acid derivative is required for preparation of the starting material as mentioned above, ${ }^{3 \mathrm{~b}, 6}$ we now wish to report a new and effective stereoselective synthesis of $(E)-\alpha, \beta$-didehydroamino acid derivative 1 via $c i s-4,5$-oxazolidinone derivative 2 starting from methyl $N$-Boc- $N$-phenoxycarbonylglycinate $\mathbf{3 f}$ and a variety of aldehydes $\mathbf{4}$ according to retrosynthetic analysis as shown in Scheme 1. The present method is characterized by stereospecific formation of erythro- $\beta$-hydroxy- $\alpha$-amino acid derivative in situ through aldol reaction of $\mathbf{3 f}$ with a variety of aldehydes 4 , simultaneous conversion to $c i s-4,5$-oxazolidinone derivative 2 , which is stereoselectively transformed into $(E)$ $\alpha, \beta$-didehydroamino acid derivative $\mathbf{1}$, and the simplicity of experimental procedure for the preparation.

First, we prepared methyl N,N-diprotected glycinates 3a-3f by the reaction of methyl $\mathrm{N}$-monoprotected glycinate $\mathbf{5 a}-\mathbf{5 f}$ with di-t-butyl dicarbonate $\left(\mathrm{Boc}_{2} \mathrm{O}\right)$. Methyl Boc-glycinate (5a) was treated with 2 molar equivalents of $\mathrm{Boc}_{2} \mathrm{O}$ in the presence of 0.3 molar equivalents of 4-(dimethylamino)pyridine (DMAP) in dry acetonitrile to afford the desired methyl $N, N$ bis(t-butoxycarbonyl)glycinate $(\mathbf{3 a})^{7}$ in quantitative yield (Entry 1 in Table 1). Similarly, methyl N,N-diprotected glycinates $\mathbf{3 b}-\mathbf{3 f}$ were also prepared in good yields as shown in Table 1 (Entries 2-6).

Next, formation of cis-4,5-oxazolidinone derivatives through the reaction of ester enolates of $\mathbf{3 a - 3 \mathbf { f }}$ with benzaldehyde (4i) was examined as a preliminary experiment. The results are

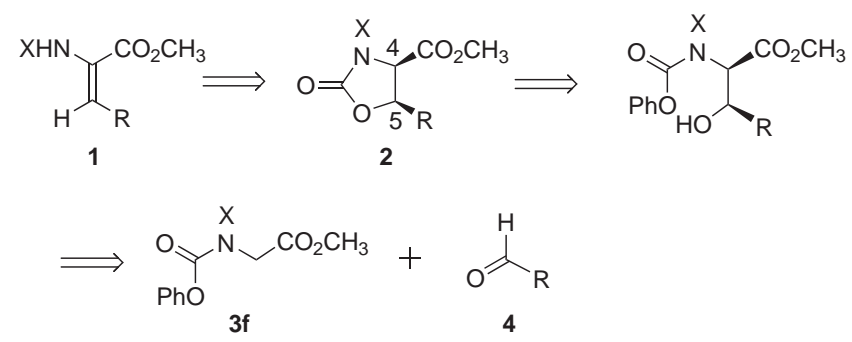

Scheme 1. Retrosynthetic analysis of $(E)$-dehydroamino acid derivative. 
Table 1. Preparation of Various N,N-Diprotected Glycine Methyl Esters

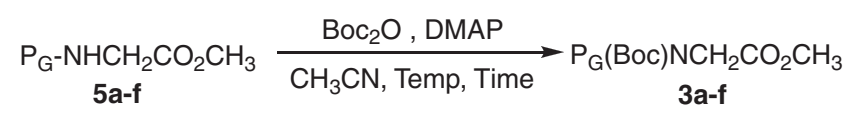

\begin{tabular}{|c|c|c|c|c|c|c|c|c|}
\hline \multirow{2}{*}{ Entry } & \multirow{2}{*}{ Substrate } & \multirow{2}{*}{$\mathrm{P}_{\mathrm{G}}$} & \multirow{2}{*}{$\begin{array}{c}\mathrm{Boc}_{2} \mathrm{O} \\
/ \text { mol equiv }\end{array}$} & \multirow{2}{*}{$\begin{array}{c}\text { DMAP } \\
/ \text { mol equiv }\end{array}$} & \multicolumn{2}{|c|}{ Reaction conditions } & \multirow{2}{*}{\multicolumn{2}{|c|}{ Product $/ \%$}} \\
\hline & & & & & $\mathrm{Temp} /{ }^{\circ} \mathrm{C}$ & Time/h & & \\
\hline 1 & $5 \mathbf{5 a}$ & Boc & 2.0 & 0.3 & 55 & 22 & $3 \mathbf{a}$ & quant \\
\hline 2 & $5 \mathbf{b}$ & $\mathrm{Z}$ & 1.5 & 0.2 & $\mathrm{rt}$ & 2 & $\mathbf{3 b}$ & 77 \\
\hline 3 & $5 c$ & $\mathrm{Cl}_{3} \mathrm{CH}_{2} \mathrm{CO}_{2} \mathrm{C}$ & 1.1 & 0.1 & $\mathrm{rt}$ & 0.3 & $3 c$ & 98 \\
\hline 4 & 5d & $\mathrm{H}_{3} \mathrm{CO}_{2} \mathrm{C}$ & 1.5 & 0.2 & $\mathrm{rt}$ & 2 & 3d & 90 \\
\hline 5 & $5 e$ & $\mathrm{H}_{3} \mathrm{CH}_{2} \mathrm{CO}_{2} \mathrm{C}$ & 1.2 & 0.1 & $\mathrm{rt}$ & 0.3 & $3 e$ & quant \\
\hline 6 & $5 f$ & $\mathrm{PhO}_{2} \mathrm{C}$ & 1.1 & 0.1 & -5 & 3 & $3 f$ & quant \\
\hline
\end{tabular}

Table 2. Preparation of cis-4,5-Oxazolidinone Derivatives

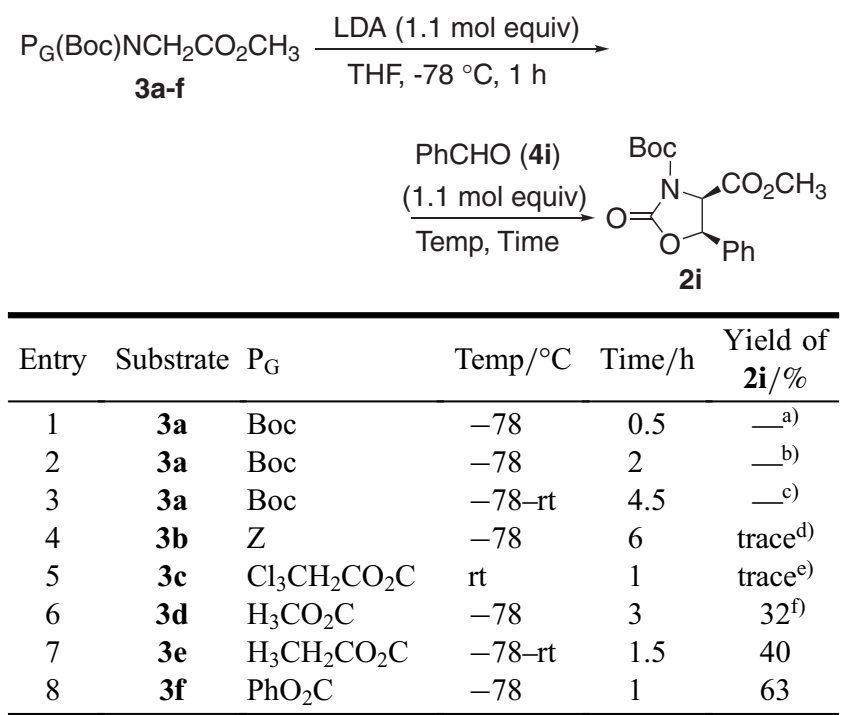

a) Methyl 2-(t-butoxycarbonylamino)-3-(t-butoxycarbonyloxy)3-phenylpropanoate (6) was obtained in $85 \%$ yield. b) $56 \%$ of compound 6 and $11 \%$ of methyl (Z)- $N$-Boc- $\alpha, \beta$-didehydrophenylalaninate $\left(\mathbf{1} \mathbf{i}^{\prime}\right)$ were obtained. c) A mixture of $\mathbf{1} \mathbf{i}^{\prime}$ and methyl (E)- $N$-Boc- $\alpha, \beta$-didehydrophenylalaninate (1i) was obtained in $36 \%$ and $25 \%$ yields, respectively. d) Recovery of $\mathbf{3 b}$ in $38 \%$ yield. e) Recovery of $\mathbf{3 c}$ in $45 \%$ yield along with $37 \%$ of 5c. f) Recovery of $\mathbf{3 d}$ in $16 \%$ yield.

summarized in Table 2. Lithiation of 3a with 1.1 molar equivalents of lithium diisopropylamide (LDA) in tetrahydrofuran (THF) at $-78^{\circ} \mathrm{C}$ for $1 \mathrm{~h}$ followed by the reaction of the lithium ester enolate thus prepared with 1.1 molar equivalents of benzaldehyde (4i) at $-78^{\circ} \mathrm{C}$ for $30 \mathrm{~min}$ did not afford the desired product $\mathbf{2 i}$, but methyl 2-( $t$-butoxycarbonylamino)-3( $t$-butoxycarbonyloxy)-3-phenylpropanoate (6) in $85 \%$ yield (Entry 1 in Table 2). Although the substrates $\mathbf{3 b}$ and $\mathbf{3 c}$ were treated with benzaldehyde (4i) under similar reaction conditions, no product $2 \mathbf{i}$ was obtained (Entries 4 and 5 in Table 2). A plausible reaction mechanism for formation of $\mathbf{6}$ and methyl (Z)- or (E)- $N$-Boc- $\alpha, \beta$-didehydrophenylalaninate $\left(\mathbf{1} \mathbf{i}^{3 \mathrm{~b}, 3 \mathrm{~h}, 8}\right.$ and $\left.\mathbf{1 i}\right)$ may be explained as follows: the lithium ester enolate derived from 3a was reacted with benzaldehyde (4i) to afford the lithium alkoxide of methyl $N, N$-diBoc- $\beta$-phenylserinate. Subsequently, the lithium alkoxide anion attacked the carbonyl carbon atom of the $N$-Boc group resulting in formation of $\mathbf{6}$ through a five-membered cyclic intermediate. Namely, it reveals that $\mathrm{N}, \mathrm{O}$ migration of the Boc group took place during the reaction as shown in Scheme 2. Accordingly, in Entry 1, quenching the reaction mixture afforded only 6 in $85 \%$ yield, while a mixture of $\mathbf{6}$ and $\mathbf{1} \mathbf{i}^{\prime}$ due to elimination of carbon dioxide and lithium tert-butoxide for longer reaction time as shown in Entry 2, and a mixture of $\mathbf{1 i}$ and $\mathbf{1} \mathbf{i}^{\prime}$ at elevated temperature in Entry 3 (in Table 2) were furnished, respectively. Reversibly, in the cases of the substrates $\mathbf{3 d}-\mathbf{3 f}$, the desired product $2 \mathbf{i}$ as a single isomer was obtained in $32 \%$, $40 \%$, and $63 \%$ yields, respectively (Entries 6-8 in Table 2). Although the difference between the results using $\mathbf{3 a - 3 f}$ is now not clear, the result in Entry 8 may be attributed to stabilities of $\mathbf{3 f}$ under these reaction conditions and of the leaving phenoxide anion. The structure of product $\mathbf{2 i}$ was confirmed by comparison of its ${ }^{1} \mathrm{HNMR}$ data with those of the known compound reported by Naito et al. ${ }^{9 a}$ and NOE measurement. The chemical shifts and coupling constant $(J=8.90 \mathrm{~Hz})$ between the hydrogens at the C4-C5 position of $\mathbf{2} \mathbf{i}$ are fully in agreement with those of the reported values $(J=9.0 \mathrm{~Hz}){ }^{9 \mathrm{a}, 9 \mathrm{~b}}$ Eventually, it was found that the stereochemical relationship between the hydrogens at the $\mathrm{C} 4-\mathrm{C} 5$ position is a cis configuration. As compound $\mathbf{3 f}$ among $\mathbf{3 a - 3 f}$ examined was found to be a useful starting material for construction of the cis-4,5-oxazolidinone system, the reaction of compound $3 f$ with a variety of aldehydes $4 a-4 h$ and $4 \mathbf{j}-\mathbf{4 l}$ was examined. The results are listed in Table 3 . When the lithium ester enolate derived from $3 f$ at $-40^{\circ} \mathrm{C}$ for $1 \mathrm{~h}$ in THF was reacted with 1.1 molar equivalents of acetaldehyde (4a) at $-40^{\circ} \mathrm{C}$ for $4 \mathrm{~h}$, no product was obtained at all (Entry 1 in Table 3). After formation of the lithium ester enolate of $\mathbf{3 f}$ at $-78^{\circ} \mathrm{C} 4 \mathrm{a}$ was reacted at -40 or $-78^{\circ} \mathrm{C}$ for $4 \mathrm{~h}$ to furnish the desired product $\mathbf{2 a}$ in moderate yields (Entries 2 and 3 in Table 3). In a similar manner, the products $\mathbf{2 b}$ and $\mathbf{2 c}$ were obtained in moderate yields by treating the reaction mixture at -78 to $-40^{\circ} \mathrm{C}$ for $4 \mathrm{~h}$ (Entries 4 and 5 in Table 3 ). In the absence of additive, $\mathbf{2 e}, \mathbf{2 g}$, and $\mathbf{2 h}$ were produced only in $43 \%$, $42 \%$, and $31 \%$ yields, respectively (Entries 7, 9, and 10). In order to improve the yields by activation of the aldehydes $\mathbf{4 e}$, 4g, and $4 \mathbf{h}$ Lewis acids such as $\mathrm{Ti}\left(\mathrm{O}^{i} \mathrm{Pr}\right)_{4}$ and $\mathrm{Cl}_{2} \mathrm{Ti}\left(\mathrm{O}^{i} \mathrm{Pr}\right)_{2}$ were used as an additive to produce the corresponding $\mathbf{2 e}, \mathbf{2 g}$, and $\mathbf{2 h}$ in $66 \%, 51 \%$, and $45 \%$ yields, respectively (Entries 7, 9, and 10 in Table 3 ). In the cases of aromatic aldehydes $\mathbf{4 j - 4 l}$ the desired products $\mathbf{2} \mathbf{j}-\mathbf{2 l}$ were also obtained in moderate yields (Entries 11-13 in Table 3). 


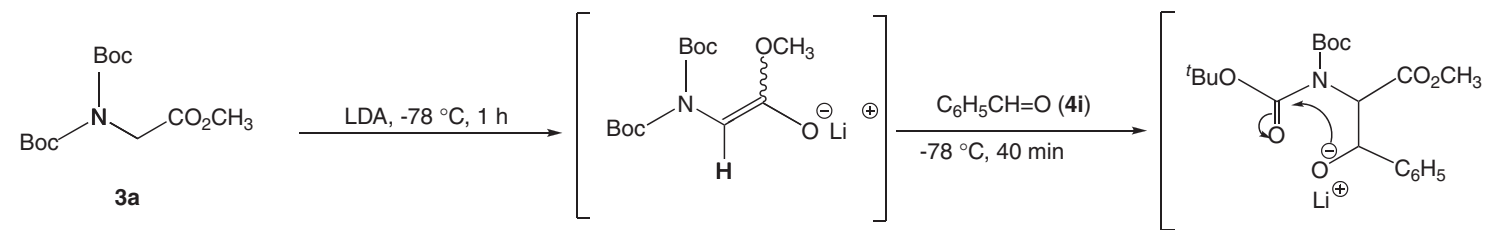

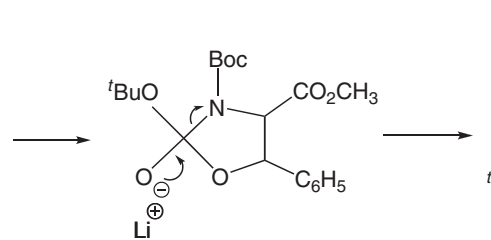<smiles>C=NC(C(=O)OCc1ccccc1)C(OC(C)=O)C(=O)OCC</smiles>

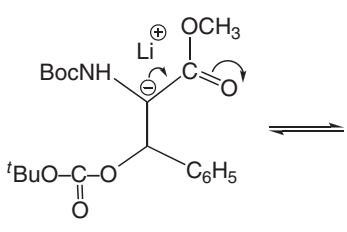

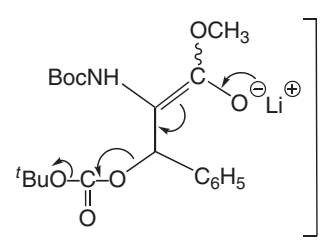<smiles>CC(=O)OC(C)C(NC(=O)OCc1ccccc1)C(=O)O</smiles><smiles>CCCCC(=O)OCC(=O)OCc1ccccc1</smiles>

$(E)-1 \mathrm{i},(Z)-1 i^{\prime}$

Scheme 2.

Table 3. Preparation of Various Oxazolidinone Derivatives

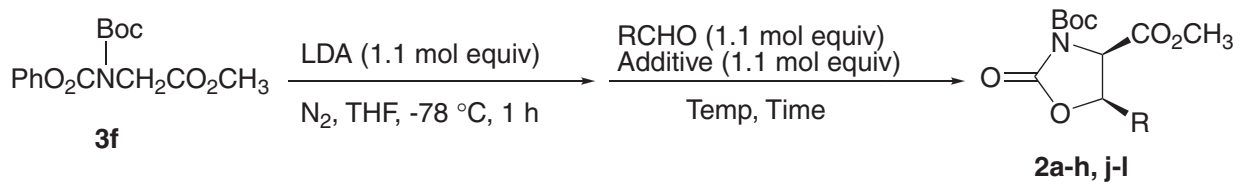

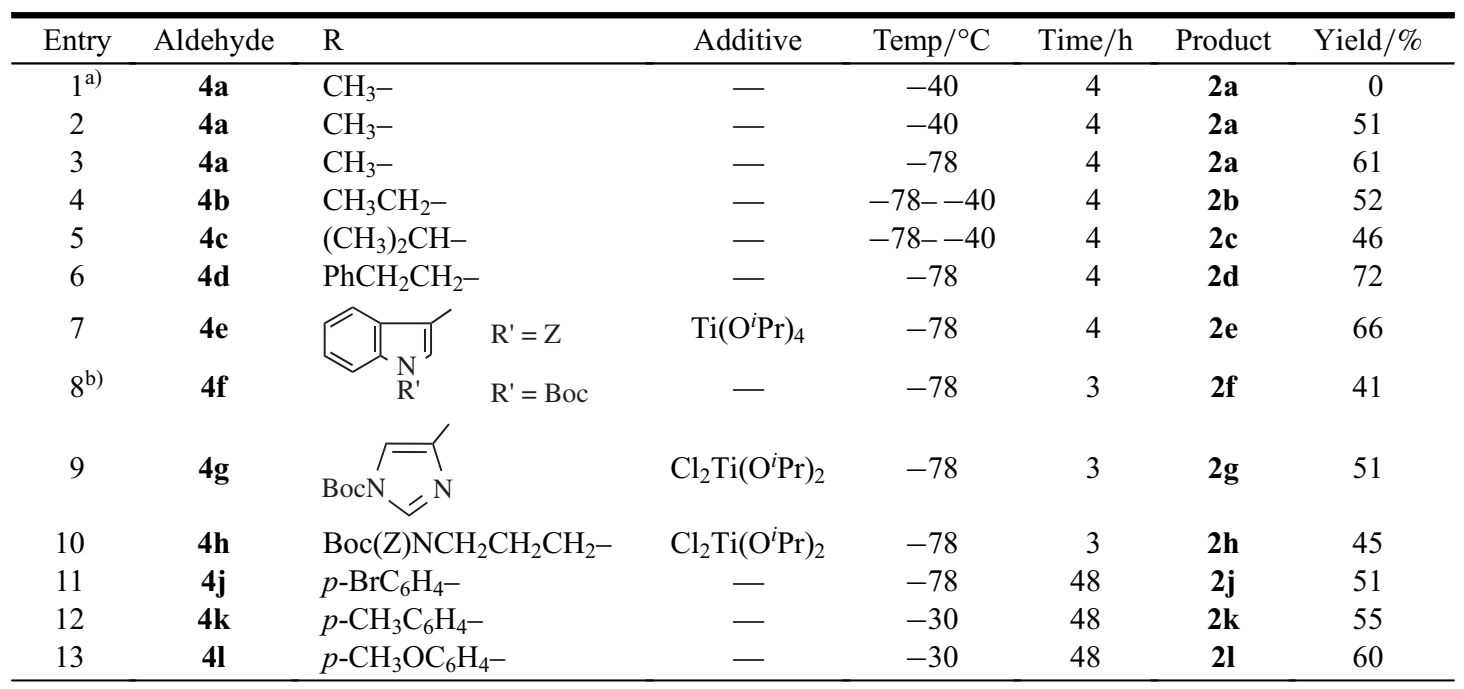

a) The lithium ester enolate was prepared at $-40{ }^{\circ} \mathrm{C}$. b) LiHMDS was used as base.

Stereoselective formation of 4,5-cis-oxazolidinone derivative 2 might be explained as follows. The silyl enol ester and the O-alkylated enol ester were first isolated to determine configuration of the lithium ester enolate formed in the solution. Namely, the lithium ester enolate derived from 3a or $3 f$ with LDA at $-78^{\circ} \mathrm{C}$ in THF was treated with excess of chlorotrimethylsilane, $t$-butyldimethylsilyl trifluoromethanesulfonate, $t$-butyldiphenylsilyl chloride (TBDPS-Cl), or benzyl bromide. $^{10}$ Unfortunately all attempts failed. The reaction might proceed via TS 1 with the least steric hindrance among
TS 1 to TS 4 to afford the lithium alkoxide of the corresponding erythro- $\beta$-hydroxy- $\alpha$-amino acid ester. ${ }^{11}$ Subsequently, nucleophilic attack of the alkoxide anion thus formed onto the carbonyl carbon atom of the $\mathrm{N}$-phenoxycarbonyl group might proceed and the subsequent elimination of the lithium phenoxide gives the desired cis-4,5-oxazolidinone derivative 2 as shown in Scheme 3.

As synthetic route to cis-4,5-oxazolidinone derivatives $\mathbf{2 a - 2 l}$ starting from $\mathbf{3 f}$ and aldehydes $\mathbf{4}$ was achieved, conversion of diastereomerically pure compounds $\mathbf{2 a - 2 l}$ into $(E)-\alpha, \beta$-dide- 


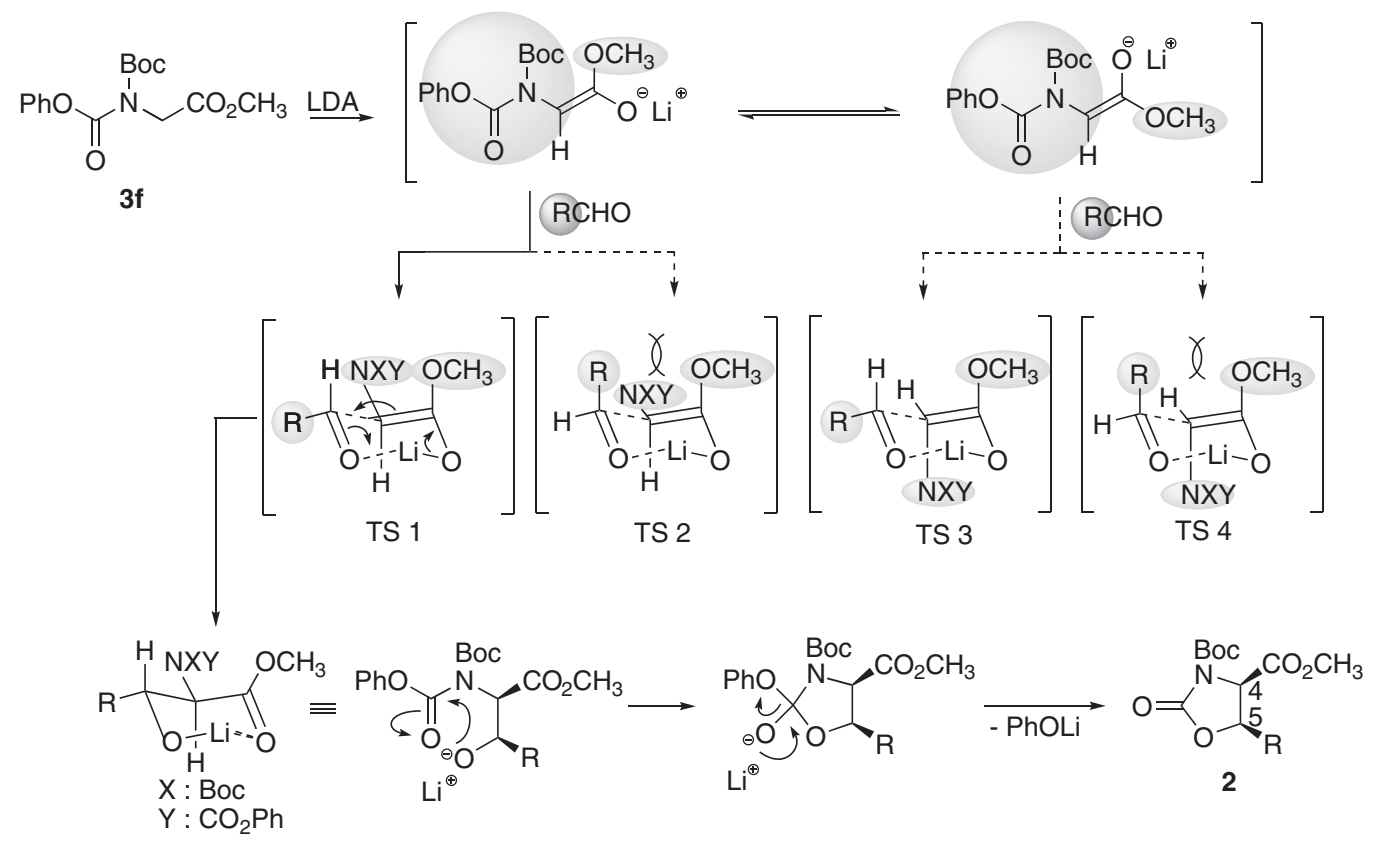

Scheme 3. A plausible reaction mechanism.

Table 4. Conversion of Oxazolidinone Derivatives to (E)-Dehydroamino Acid Derivatives

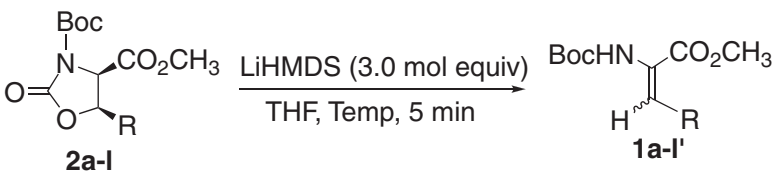

\begin{tabular}{|c|c|c|c|c|c|c|c|}
\hline \multirow{3}{*}{$\begin{array}{c}\text { Entry } \\
1\end{array}$} & \multirow{3}{*}{$\begin{array}{c}\text { Substrate }^{\mathrm{a})} \\
\mathbf{2 a}\end{array}$} & \multirow{2}{*}{$\mathrm{R}$} & \multirow{2}{*}{ Temp $/{ }^{\circ} \mathrm{C}$} & \multicolumn{4}{|c|}{ Yield $/ \%$} \\
\hline & & & & \multicolumn{2}{|c|}{ E form } & \multicolumn{2}{|c|}{$\mathrm{Z}$ form } \\
\hline & & $\mathrm{CH}_{3}-$ & -40 & $1 \mathbf{a}$ & 57 & $1 a^{\prime}$ & 41 \\
\hline $2^{\mathrm{b})}$ & $\mathbf{2 a}$ & $\mathrm{CH}_{3}-$ & -78 & $1 \mathbf{a}$ & 68 & $1 a^{\prime}$ & 27 \\
\hline $3^{\mathrm{c})}$ & $2 a$ & $\mathrm{CH}_{3}-$ & -100 & $1 \mathbf{a}$ & 76 & $1 a^{\prime}$ & $<24$ \\
\hline 4 & $2 a$ & $\mathrm{CH}_{3}-$ & -78 & $1 \mathbf{a}$ & 85 & $1 a^{\prime}$ & $<13$ \\
\hline 5 & $2 \mathbf{b}$ & $\mathrm{CH}_{3} \mathrm{CH}_{2-}$ & -78 & $1 b$ & 91 & $1 b^{\prime}$ & $<9$ \\
\hline 6 & $2 c$ & $\left(\mathrm{CH}_{3}\right)_{2} \mathrm{CH}-$ & -78 & $1 \mathrm{c}$ & 96 & $1 c^{\prime}$ & $<4$ \\
\hline 7 & 2d & $\mathrm{PhCH}_{2} \mathrm{CH}_{2-}$ & -78 & $1 d$ & 96 & $1 d^{\prime}$ & $<4$ \\
\hline $8^{\text {d) }}$ & $2 e$ & $\mathrm{R}^{\prime}=\mathrm{Z}$ & -10 & $1 \mathrm{e}$ & 93 & $1 \mathrm{e}^{\prime}$ & $<7$ \\
\hline 9 & $2 f$ & $\mathrm{R}^{\prime}=\mathrm{Boc}$ & -78 & 1f & 82 & $1 \mathbf{f}^{\prime}$ & $<8$ \\
\hline 10 & $2 \mathrm{~g}$ & $=$ & -10 & $1 \mathrm{~g}$ & 48 & $1 g^{\prime}$ & 25 \\
\hline 11 & $2 \mathrm{~g}$ & $\mathrm{BocN}=\mathrm{N}$ & -78 & $1 g$ & 83 & $1 g^{\prime}$ & $<4$ \\
\hline 12 & $2 \mathrm{~h}$ & $\mathrm{Boc}(\mathrm{Z}) \mathrm{NCH}_{2} \mathrm{CH}_{2} \mathrm{CH}_{2}-$ & -10 & $1 \mathrm{~h}$ & 62 & $1 h^{\prime}$ & 25 \\
\hline 13 & $2 \mathbf{i}$ & $\mathrm{Ph}-$ & -20 & $\mathbf{1 i}$ & 92 & $1 i^{\prime}$ & 6 \\
\hline 14 & $2 \mathbf{j}$ & $p-\mathrm{BrC}_{6} \mathrm{H}_{4-}$ & -20 & $\mathbf{1 j}$ & 89 & $1 j^{\prime}$ & 9 \\
\hline 15 & $2 k$ & $p-\mathrm{CH}_{3} \mathrm{C}_{6} \mathrm{H}_{4}-$ & -20 & $1 \mathrm{k}$ & 92 & $1 k^{\prime}$ & 6 \\
\hline 16 & 21 & $p-\mathrm{CH}_{3} \mathrm{OC}_{6} \mathrm{H}_{4-}$ & -20 & 11 & 91 & $11^{\prime}$ & 7 \\
\hline
\end{tabular}

a) Diastereomerically pure substrates $\mathbf{2 a}-\mathbf{2 l}$ were used. b) HMPA (3.0 mol equiv) was added. c) Reacted for 30 min. d) Reacted for $10 \mathrm{~min}$.

hydroamino acid derivatives $\mathbf{1 a - 1 1}$ was examined under basic conditions. The results are listed in Table 4. Various bases such as 1,4-diazabicyclo[2.2.2] octane (DABCO), 1,8-diazabicyclo[5.4.0] undec-7-ene (DBU), $\mathrm{KO}^{t} \mathrm{Bu}, \mathrm{KOH}$, and lithium hexamethyldisilazide (LiHMDS) in THF were used. Eventually, LiHMDS was base of choice among bases tested in THF to afford $\alpha, \beta$-didehydroamino acid derivatives $\mathbf{1 a - 1 1}$ predominantly accompanied by $\alpha, \beta$-didehydroamino acid derivatives $\mathbf{1} \mathbf{a}^{\prime}-\mathbf{1} \mathbf{l}^{\prime}$. Compound $\mathbf{2 a}$ was treated with 3.0 molar equivalents of LiHMDS at $-40{ }^{\circ} \mathrm{C}$ for $5 \mathrm{~min}$ to afford methyl 2-( $t$-butoxycarbonylamino)-2-butenoate as a separable mixture of $\mathbf{1 a}$ and $\mathbf{1} \mathbf{a}^{\prime}$ in $57 \%$ and $41 \%$ yields, respectively (Entry 1 in Table 4). 

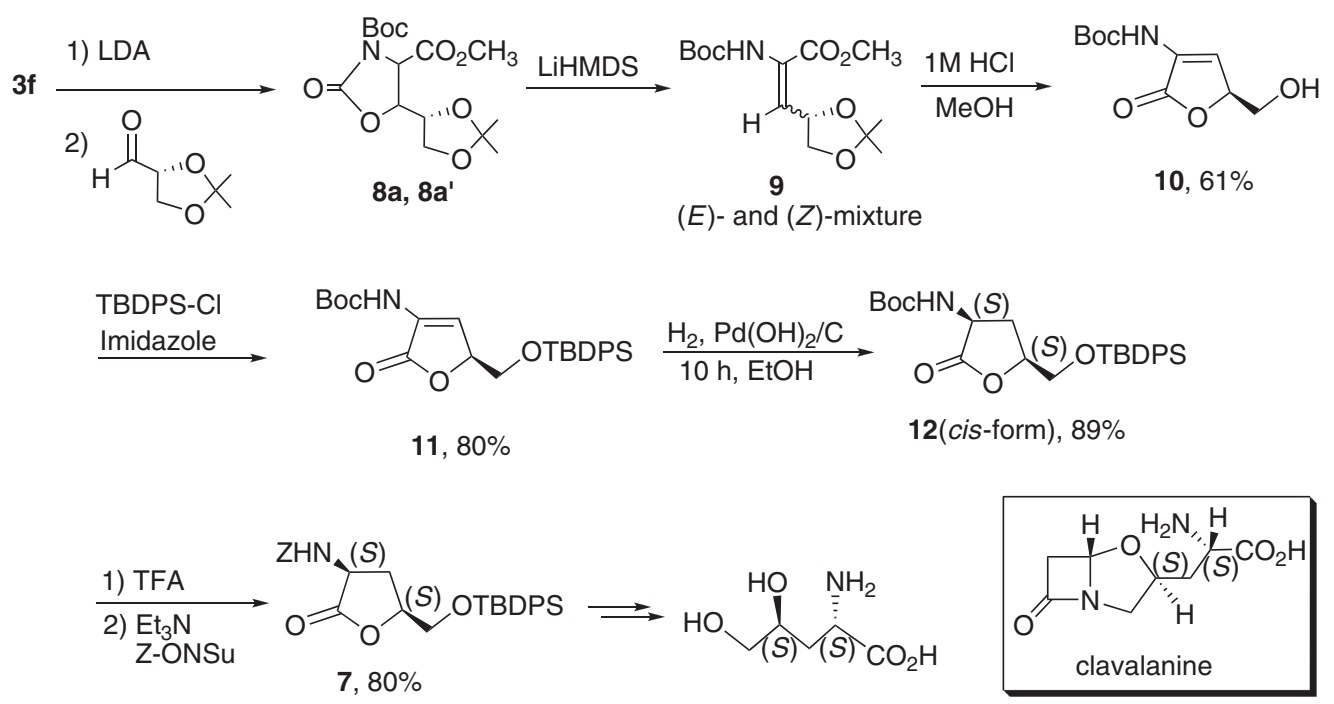

Scheme 4. Synthesis of (3S,5S)-3-(Z-amino)-5-(TBDPS-oxymethyl)- $\gamma$-lactone 7.

Major product 1a was assigned to be (E)-2-( $t$-butoxycarbonylamino)-2-butenoate by comparison with the reported ${ }^{1} \mathrm{HNMR}$ data $^{3 \mathrm{~b}}$ and by NOE measurement. Minor product $1 \mathbf{a}^{\prime}$ proved to be methyl (Z)-2-( $t$-butoxycarbonylamino)-2-butenoate by comparison with the reported ${ }^{1} \mathrm{HNMR}$ data. ${ }^{3 \mathrm{~b}, 3 \mathrm{c}, 3 \mathrm{i}, 8 \mathrm{a}}$ On treatment of $2 \mathbf{a}$ with 3.0 molar equivalents of LiHMDS at $-100^{\circ} \mathrm{C}$ for $30 \mathrm{~min}$, stereoselectivity of $\mathrm{E}$ and $\mathrm{Z}$ isomers $\left(\mathbf{1} \mathbf{a} / \mathbf{1} \mathbf{a}^{\prime}\right)$ was improved considerably (Entry 3 in Table 4). Moreover, 2a was reacted at $-78^{\circ} \mathrm{C}$ for $5 \mathrm{~min}$ to afford compound 1a in $85 \%$ yield (Entry 4). Predominant formation of 1a from $2 \mathbf{a}$ might be due to trans elimination by deprotonation with base at the $\alpha$ position of the ester group. ${ }^{3 \mathrm{~h}}$ In a similar manner, compounds $(E)-\mathbf{1 b}-\mathbf{1 l}$ were stereoselectively prepared in moderate to high yields (Entries 5-16). Stereochemistry of $\mathbf{1 b}, \mathbf{1 d}-\mathbf{1 g}$, and $\mathbf{1 i}-\mathbf{1 l}$ were assigned by NOE measurement without $\mathbf{1 c}$ and $\mathbf{1 h}$, whose stereochemistry were determined by isomerization to the corresponding $\mathbf{1} \mathbf{c}^{\prime}$ and $\mathbf{1} \mathbf{h}^{\prime}$ using a catalytic amount of $\mathrm{I}_{2}$ (Entries 6 and 12 in Table 4). ${ }^{12}$ The ${ }^{1} \mathrm{HNMR}$ spectra of $\mathbf{1 a}^{\prime},{ }^{3 b, 3 c, 3 i, 8 a} \mathbf{1 b}^{\prime},{ }^{8 a} \mathbf{1 c}^{\prime},{ }^{8 a} \mathbf{1 d}^{\prime},{ }^{13} \mathbf{1 i}^{\prime},{ }^{3 b, 8 a-8 c} \mathbf{1 k}^{\prime},{ }^{14}$ and $\mathbf{1}^{\prime 15}$ were satisfactorily in accordance with the reported data of $\mathrm{Z}$ isomer.

Because it was found that compounds 2 could be stereoselectively converted into $(E)-\alpha, \beta$-didehydroamino acid derivatives 1 the present method was employed for synthesis of $(3 S, 5 S)$-3-(benzyloxycarbonylamino)-5-( $t$-butyldiphenylsiloxy)- $\gamma$-lactone (7), ${ }^{16}$ known as a key synthetic intermediate of the antibiotic clavalanine ${ }^{17}$ isolated from streptomyces clavuligerus according to Scheme 4. First, compound 8 was prepared as the starting material as shown in Table 5. After $\mathbf{3 f}$ was treated with 1.1 molar equivalents of LDA at $-78^{\circ} \mathrm{C}$ for $30 \mathrm{~min}$, the resulting lithium ester enolate was reacted with 1.5 molar equivalents of $(R)-O-2,3$-isopropylideneglycelaldehyde at $-78^{\circ} \mathrm{C}$ for $4 \mathrm{~h}$ to afford a separable mixture of two diastereomers, of which $\mathbf{8 a}$ was produced as an oil and $\mathbf{8} \mathbf{a}^{\prime}$ in crystalline form in $22 \%$ and $30 \%$ yields, respectively (Entry 1 in Table 5). As $15.2 \%$ and $11.4 \%$ NOE between the hydrogens at $\mathrm{C} 4-\mathrm{C} 5$ in irradiation of the proton at $\mathrm{C} 4$ in products $8 \mathbf{a}$ and $\mathbf{8} \mathbf{a}^{\prime}$ were observed, the stereochemistry between the hydrogens at $\mathrm{C} 4-\mathrm{C} 5$ of the oxazolidinone ring is assigned to be cis configuration. To improve the total yield various reaction
Table 5. Preparation of Oxazolidinone 8

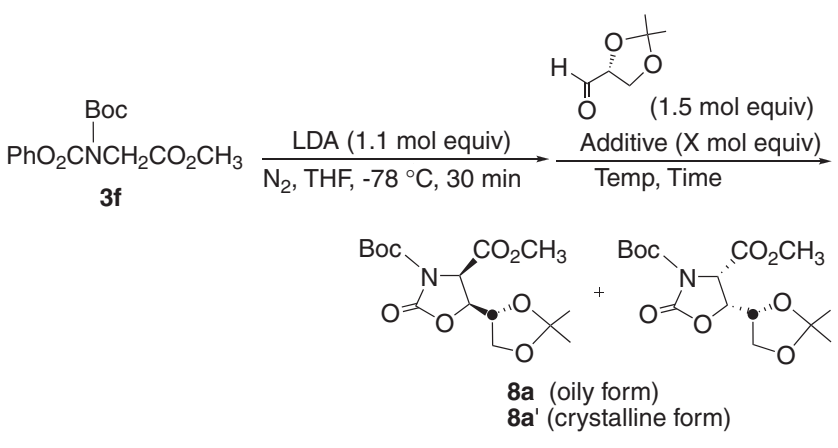

\begin{tabular}{|c|c|c|c|c|c|c|}
\hline \multirow{2}{*}{ Entry } & \multirow{2}{*}{ Additive } & \multirow{2}{*}{$\begin{array}{c}X \\
/ \text { mol equiv }\end{array}$} & \multirow{2}{*}{$\begin{array}{l}\text { Temp } \\
/{ }^{\circ} \mathrm{C}\end{array}$} & \multirow{2}{*}{$\begin{array}{c}\text { Time } \\
/ \mathrm{h}\end{array}$} & \multicolumn{2}{|c|}{ Yield/\% } \\
\hline & & & & & $8 \mathbf{a}$ & $\mathbf{8 a ^ { \prime }}$ \\
\hline 1 & - & - & -78 & 4 & 22 & 30 \\
\hline 2 & - & - & $-78--10$ & 3 & 10 & 24 \\
\hline 3 & - & - & $-78--20$ & 24 & $<22$ & $<28$ \\
\hline 4 & $\mathrm{TiCl}_{4} \cdot 2 \mathrm{THF}$ & 1.5 & -78 & 2 & 26 & - \\
\hline 5 & $\mathrm{Ti}\left(\mathrm{O}^{i} \mathrm{Pr}\right)_{4}$ & 0.5 & -78 & 2 & 24 & 20 \\
\hline 6 & $\mathrm{Ti}\left(\mathrm{O}^{i} \mathrm{Pr}\right)_{4}$ & 0.5 & -78 & 4 & $<28$ & 13 \\
\hline 7 & $\mathrm{Ti}\left(\mathrm{O}^{i} \mathrm{Pr}\right)_{4}$ & 1.1 & -78 & 4 & 32 & 16 \\
\hline 8 & $\mathrm{Ti}\left(\mathrm{O}^{i} \mathrm{Pr}\right)_{4}$ & 1.1 & -78 & 6 & 30 & 21 \\
\hline 9 & $\mathrm{Ti}\left(\mathrm{O}^{i} \mathrm{Pr}\right)_{4}$ & 1.5 & -78 & 2 & 40 & $<25$ \\
\hline 10 & $\mathrm{Ti}\left(\mathrm{O}^{i} \mathrm{Pr}\right)_{4}$ & 2.0 & -78 & 4 & 38 & 19 \\
\hline
\end{tabular}

conditions were examined. Accordingly, the ratio of two diastereomers was found to be dependent on reaction conditions (Entries 2-10 in Table 5).

Each of diastereomerically pure compounds $\mathbf{8 a}$ and $\mathbf{8 \mathbf { a } ^ { \prime }}$ thus obtained was individually exposed to the conditions for preparation of $\alpha, \beta$-didehydroamino acid via elimination of carbon dioxide as described for preparation of compound $\mathbf{1}$. The results are summarized in Table 6 . Thus, treatment of $\mathbf{8 a}$ (oily form) with 3.0 molar equivalents of LiHMDS at $-10^{\circ} \mathrm{C}$ for 5 min furnished compound 9 in a ratio of 78:22 as a mixture of $\mathrm{E}$ and $\mathrm{Z}$ isomers ${ }^{18}$ in $70 \%$ yield (Entry 2 in Table 6). Similarly, 8a' (crystalline form) was reacted with 3.0 molar equivalents of LiHMDS at $-30^{\circ} \mathrm{C}$ for $90 \mathrm{~min}$ in THF to afford 
the desired product 9 in a ratio of $85: 15$ as a mixture of $E$ and $Z$ isomers in $87 \%$ yield (Entry 5 in Table 6). Formation of a mixture of $(E)$ - and (Z)-9 might be explained as follows: the carbanion generated by deprotonation with LiHMDS undergoes trans elimination of carbon dioxide with retention of stereochemistry to afford $(E)-\mathbf{9}$, however, $(Z)-\mathbf{9}$ is produced due to formation of the ester enolate.

Next, conversion of the mixture of diastereomers 9 into compound 7 was performed according to Scheme 4 . Treatment of the mixture of diastereomers $9(\mathrm{E} / \mathrm{Z}=78: 22)$ with $1 \mathrm{M} \mathrm{HCl}$ in $\mathrm{MeOH}$ at room temperature for 1 day gave the desired $\gamma$ butenolide derivative $\mathbf{1 0}^{19}$ and methyl (4S)-(Z)-2-( $N$-Bocamino)-4,5-dihydroxy-2-pentenoate in $61 \%$ and $19 \%$ yields, respectively. Formation of the latter product was probably attributed to deacetalization of the starting $\mathrm{Z}$ isomer 9 since the authentic $\mathrm{Z}$ isomer 9 prepared by an alternative way ${ }^{5}$ afforded only the deacetalization product in $90 \%$ yield under the same reaction conditions. Therefore, $\mathrm{E}$ isomer 9 was cyclized to the oxazolidinone derivative 10, whereas $\mathrm{Z}$ isomer 9 underwent deacetalization. Next, compound $\mathbf{1 0}$ was reacted with 1.2 molar equivalents of TBDPS-Cl in the presence of 3 molar equivalents of imidazole at room temperature for $4 \mathrm{~h}$ in dimethylformamide (DMF) to afford O-protected product $\mathbf{1 1}$ in $80 \%$ yield. Hydrogenation of the resulting compound $\mathbf{1 1}$

Table 6. Conversion of Oxazolidinone 8 to Dehydroamino Acid Derivative 9

8a (oily form) or
8a' (crystalline form)

\begin{tabular}{cccccc}
\hline Entry & Substrate $^{\mathrm{a})}$ & $\mathrm{Temp} /{ }^{\circ} \mathrm{C}$ & $\mathrm{Time} / \mathrm{min}$ & Yield/\% & $\mathrm{E} / \mathrm{Z}$ \\
\hline 1 & $\mathbf{8 a}$ & -10 & 3 & 50 & $76 / 24$ \\
2 & $\mathbf{8 a}$ & -10 & 5 & 70 & $78 / 22$ \\
3 & $\mathbf{8 a}$ & -30 & 60 & 61 & $77 / 23$ \\
4 & $\mathbf{8 a}$ & -10 & 5 & 73 & $89 / 11$ \\
5 & $\mathbf{8 a}^{\prime}$ & -30 & 90 & 87 & $85 / 15$ \\
\hline
\end{tabular}

a) Stereoisomerically pure substrates $\mathbf{8 a}$ and $\mathbf{8 \mathbf { a } ^ { \prime }}$ were used. over $20 \% \mathrm{Pd}(\mathrm{OH})_{2}$-carbon in EtOH under hydrogen atmosphere at room temperature for $10 \mathrm{~h}$ took place stereospecifically from the less hindered side due to the bulky TBDPSoxymethyl substituent at position 5 to afford exclusively cis 3,5-disubstituted $\gamma$-lactone derivative $\mathbf{1 2}$ as a single isomer in $89 \%$ yield, ${ }^{5}$ whereas in the case of longer (overnight) reaction time, a mixture of cis and trans isomers was obtained in $62 \%$ and $13 \%$ yields, respectively. N-Deprotection of the resulting compound 12 by trifluoroacetic acid (TFA) at $0{ }^{\circ} \mathrm{C}$ to room temperature for $1 \mathrm{~h}$ in dichloromethane and subsequent coupling reaction with 1.1 molar equivalents of $N$-(benzyloxycarbonyloxy)succinimide (Z-ONSu) in the presence of 3 molar equivalents of triethylamine (TEA) at $0{ }^{\circ} \mathrm{C}$ to room temperature for $2 \mathrm{~h}$ in THF afforded the desired compound 7 in $80 \%$ yield.

In a previous paper, ${ }^{12}$ we reported that isomerization of dipeptide containing $(E)-N$-Boc- $\alpha, \beta$-didehydroamino acid residue to the corresponding $\mathrm{Z}$ isomer could be readily performed by means of a catalytic amount of iodine. Indeed, stereochemically pure methyl (E)-2(N-t-butoxycarbonylamino)-2-butenoate (1a) was treated with a catalytic amount of iodine at room temperature for $19 \mathrm{~h}$ in THF to furnish the corresponding $\mathrm{Z}$ isomer $\mathbf{1} \mathbf{a}^{\prime}$ in $90 \%$ yield (Entry 1 in Table 7). In a similar way, pure $\mathrm{E}$ isomers $\mathbf{1 b}-\mathbf{1 h}$ were smoothly isomerized to the corresponding $\mathrm{Z}$ isomers $\mathbf{1} \mathbf{b}^{\prime}-\mathbf{1} \mathbf{h}^{\prime}$ in high yields. The ${ }^{1} \mathrm{HNMR}$ data of the products $1 \mathbf{a}^{\prime}-\mathbf{1 h}^{\prime}$ produced by isomerization were satisfactorily identical with those of the minor products obtained in Entries 4-12 in Table 4. The results are summarized in Table 7.

We next tried to prepare the amide compounds $\mathbf{1 3 a}-\mathbf{1 3} \mathbf{k}^{\prime}$ by the reaction of diastereomerically pure cis-4,5-oxazolidinoes 2a, 2d, and $\mathbf{2 i}$ with $N$-Boc amino acid derivatives as shown in Table 8 . Thus, after $\mathrm{N}$-deprotection of $\mathbf{2} \mathbf{i}$ with $99 \%$ formic acid at room temperature for $40 \mathrm{~min}$, the resulting oxazolidinone derivative was treated with 1.1 molar equivalents of LDA at $-78^{\circ} \mathrm{C}$ for $5 \mathrm{~min}$ in dry $\mathrm{THF}$ followed by reaction with 1.1 molar equivalents of the $N$-hydroxysuccinimide (HONSu) ester of Boc-alanine at -78 to $-10^{\circ} \mathrm{C}$ for $3 \mathrm{~h}$ to afford the corresponding amide product $\mathbf{1 3} \mathbf{i}$ as a major product and $\mathbf{1 3} \mathbf{i}^{\prime}$ as a minor one in $96 \%$ total yields as a separable mixture of

Table 7. Isomerization of $\mathrm{E}$ to $\mathrm{Z}$ Isomer by Iodine

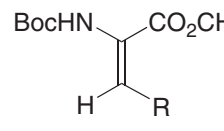

(E)-1a-h

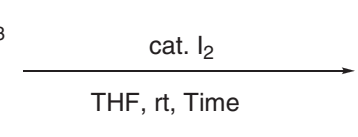

HF, rt, Time

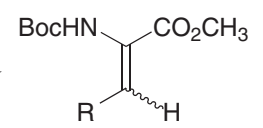

(Z)-1a'-h'

\begin{tabular}{|c|c|c|c|c|c|c|c|}
\hline \multirow{2}{*}{$\frac{\text { Entry }}{1}$} & \multirow{2}{*}{$\frac{\text { Substrate }^{\mathrm{a})}}{\mathbf{1} \mathbf{a}}$} & \multirow{2}{*}{$\frac{\mathrm{R}}{\mathrm{CH}_{3}-}$} & & \multirow{2}{*}{$\frac{\text { Time } / \mathrm{h}}{19}$} & \multicolumn{2}{|c|}{ Yield/\% } & \multirow{2}{*}{$\frac{E / Z}{4 / 96}$} \\
\hline & & & & & $1 \mathbf{a}^{\prime}$ & 90 & \\
\hline 2 & $1 b$ & $\mathrm{CH}_{3} \mathrm{C}$ & & 17 & $1 b^{\prime}$ & 91 & $3 / 97$ \\
\hline 3 & $1 c$ & $\left(\mathrm{CH}_{3}\right)$ & & 21 & $1 c^{\prime}$ & 96 & $5 / 95$ \\
\hline 4 & $1 d$ & $\mathrm{PhCH}$ & & 12 & $1 d^{\prime}$ & 98 & $3 / 97$ \\
\hline 5 & $1 e$ & & $\mathrm{R}^{\prime}=\mathrm{Z}$ & 24 & $1 \mathrm{e}^{\prime}$ & 97 & 4/96 \\
\hline 6 & $1 f$ & & $\mathrm{R}^{\prime}=\mathrm{Boc}$ & 43 & $1 \mathbf{f}^{\prime}$ & 93 & $9 / 91$ \\
\hline 7 & $1 \mathrm{~g}$ & BocN & & 16 & $1 g^{\prime}$ & quant & $0 / 100$ \\
\hline 8 & $1 \mathrm{~h}$ & $\operatorname{Boc}(Z$ & $\mathrm{CH}_{2} \mathrm{CH}_{2}-$ & 9 & $1 h^{\prime}$ & 96 & $0 / 100$ \\
\hline
\end{tabular}

a) A stereochemically pure E isomer of $\mathbf{1} \mathbf{a}-\mathbf{1 h}$ was used. 
Table 8. Coupling of Boc-Amino Acid with cis-4,5-Oxazolidinone Derivative

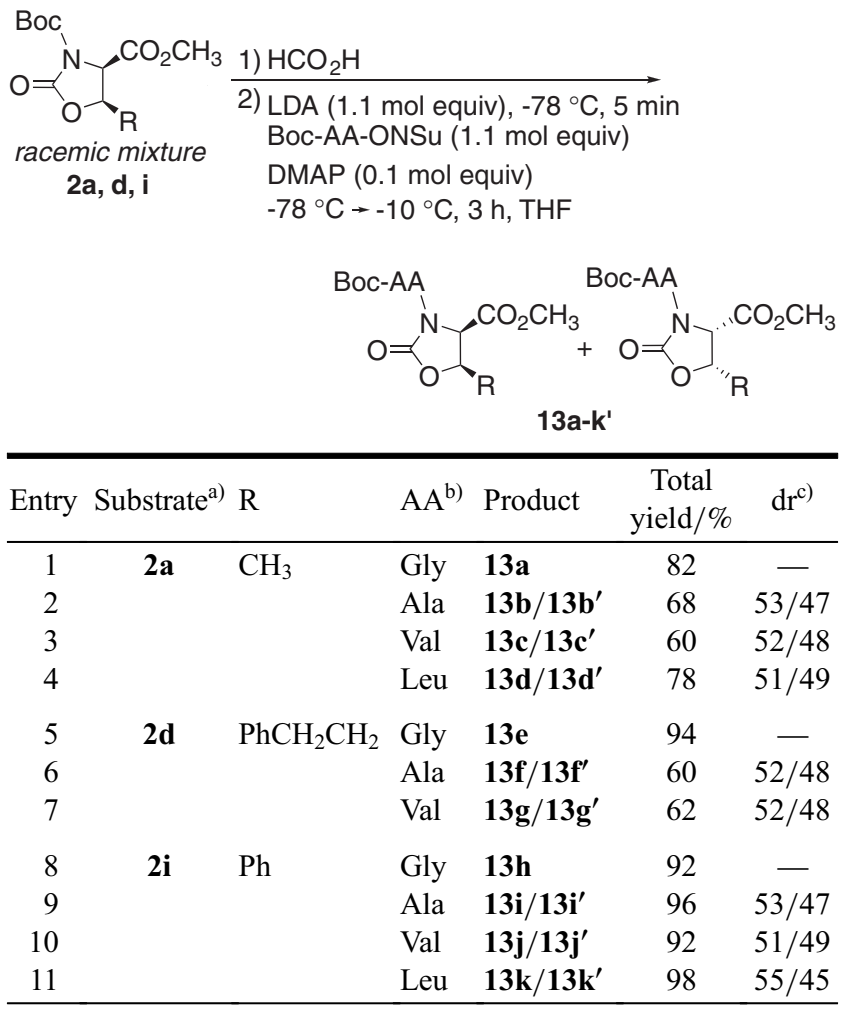

a) A diastereomerically pure isomer of $\mathbf{2 a}, \mathbf{2 d}$, and $\mathbf{2 i}$ was used. b) Means amino acid abbreviated by three letters. c) Means the ratio of the two diastereomers.

two diastereomers with a ratio of $53: 47$ as shown in Table 8 (Entry 9). Similarly, compounds 13a-13h (Entries 1-8) and $\mathbf{1 3} \mathbf{j}-\mathbf{1 3} \mathbf{k}^{\prime}$ (Entries 10 and 11) were prepared in satisfactory yields as a separable mixture of two diastereomers without Entries 1, 5, and 8 .

We next tried to prepare the dipeptide containing $(E)-\alpha, \beta$ didehydroamino acid residue as shown in Table 9. Compound 13b, the major product, thus obtained was treated with 4.0 molar equivalents of LiHMDS at $-45^{\circ} \mathrm{C}$ for $50 \mathrm{~min}$ in THF to afford the corresponding dipeptide $\mathbf{1 4 b}$ and $\mathbf{1 4} \mathbf{b}^{\prime}$ in a ratio of $84: 16$ in $88 \%$ yield (Entry 2 in Table 9). Treatment of $\mathbf{1 3 b}^{\prime}$, the minor product, with LiHMDS under the same reaction conditions also resulted in the formation of $\mathbf{1 4 b}$ and $\mathbf{1 4} \mathbf{b}^{\prime}$ in a ratio of $81: 19$ in 92\% yield (Entry 3 in Table 9). The major product 14b was found to be methyl (E)-2-(Boc-alanylamino)-2-butenoate, and $\mathbf{1 4} \mathbf{b}^{\prime}$ to be (Z)-2-(Boc-alanylamino)-2-butenoate compared with the reported data ${ }^{3 \mathrm{i}, 6 \mathrm{f}, 20}$ and NOE measurement of $\mathbf{1 4 b}$. From these results, it was found that treatment of both $\mathbf{1 3 b}$ and $\mathbf{1 3} \mathbf{b}^{\prime}$ bearing cis-4,5-oxazolidinone moiety with base afforded the dipeptide $14 \mathbf{b}$ bearing $(E)-\alpha, \beta$-didehydroamino acid residue predominantly with high stereoselectivity accompanied by $\mathrm{Z}$ isomer $\mathbf{1 4} \mathbf{b}^{\prime}$ as in the case described for conversion of $\mathbf{2}$ to $\mathbf{1}$. In the same way, compounds $14 \mathbf{a}$ and $14 \mathbf{c}-14 \mathrm{k}$ containing $\alpha, \beta$ didehydroamino acid with high $\mathrm{E}$ selectivity were prepared in good yields. The results are listed in Table 9. Stereochemistry of the major products, 14a, 14c, 14d, and $14 \mathrm{f}-14 \mathrm{k}$ was assigned to be E configuration by NOE measurement. Stereochemistry of the major product $14 \mathbf{e}$ was determined by
Table 9. Conversion of Compounds $13 \mathbf{a}-13 \mathbf{k}^{\prime}$ to the Corresponding Dehydropeptides 14a-14k'

$$
\begin{aligned}
& \text { 13a-k' } \stackrel{\text { LiHMDS (4.0 mol equiv) }}{\underset{\mathrm{THF},-45^{\circ} \mathrm{C}, 50 \mathrm{~min}}{\longrightarrow}} \stackrel{\text { Boc-AA-NH}}{\underset{\mathrm{N}}{\longrightarrow}} \\
& \text { 14a-k' }
\end{aligned}
$$

\begin{tabular}{|c|c|c|c|c|c|c|}
\hline Entry & Substrate & $\mathrm{R}$ & $\mathrm{AA}$ & Product & Yield $/ \%$ & $\mathrm{E} / \mathrm{Z}$ \\
\hline 1 & 13a & $\mathrm{CH}_{3}$ & Gly & $14 a / 14 a^{\prime}$ & 56 & $81 / 19$ \\
\hline 2 & $13 b$ & & Ala & $14 b / 14 b^{\prime}$ & 88 & $84 / 16$ \\
\hline 3 & $13 b^{\prime}$ & & Ala & $14 b / 14 b^{\prime}$ & 92 & $81 / 19$ \\
\hline 4 & $13 \mathrm{c}$ & & Val & $14 c / 14 c^{\prime}$ & 95 & $85 / 15$ \\
\hline 5 & $13 c^{\prime}$ & & Val & $14 c / 14 c^{\prime}$ & 85 & $85 / 15$ \\
\hline 6 & 13d & & Leu & $14 d / 14 d^{\prime}$ & 61 & $88 / 12$ \\
\hline 7 & $13 d^{\prime}$ & & Leu & $14 d / 14 d^{\prime}$ & 52 & $75 / 25$ \\
\hline 8 & $13 e$ & $\mathrm{PhCH}_{2} \mathrm{CH}_{2}$ & Gly & $14 \mathrm{e} / 14 \mathrm{e}^{\prime}$ & 52 & $88 / 12$ \\
\hline 9 & 13f & & Ala & $14 f / 14 f^{\prime}$ & 80 & $85 / 15$ \\
\hline 10 & $13 f^{\prime}$ & & Ala & $14 f / 14 f^{\prime}$ & 75 & $84 / 16$ \\
\hline 11 & $13 \mathrm{~g}$ & & Val & $14 \mathrm{~g} / 14 \mathrm{~g}^{\prime}$ & 72 & $89 / 11$ \\
\hline 12 & $13 g^{\prime}$ & & Val & $14 \mathrm{~g} / 14 \mathrm{~g}^{\prime}$ & 80 & $87 / 13$ \\
\hline 13 & $13 \mathrm{~h}$ & $\mathrm{Ph}$ & Gly & $14 h / 14 h^{\prime}$ & 53 & $85 / 15$ \\
\hline 14 & $13 \mathbf{i}$ & & Ala & $14 i / 14 i^{\prime}$ & 78 & $87 / 13$ \\
\hline 15 & $13 i^{\prime}$ & & Ala & $14 i / 14 i^{\prime}$ & 82 & $87 / 13$ \\
\hline 16 & $13 \mathbf{j}$ & & Val & $14 j / 14 j^{\prime}$ & 70 & $89 / 19$ \\
\hline 17 & $13 j^{\prime}$ & & Val & $14 j / 14 j^{\prime}$ & 74 & $91 / 9$ \\
\hline 18 & $13 k$ & & Leu & $14 k / 14 k^{\prime}$ & 59 & $89 / 11$ \\
\hline 19 & $13 k^{\prime}$ & & Leu & $14 k / 14 k^{\prime}$ & 60 & $88 / 12$ \\
\hline
\end{tabular}

isomerization to the minor product $14 \mathrm{e}^{\prime}$ by means of $\mathrm{I}_{2} \cdot{ }^{12}$ The minor products, $\mathbf{1 4} \mathbf{a}^{\prime},{ }^{3 \mathrm{~h}, 3 \mathrm{i}, 20} \mathbf{1 4 h}^{\prime},{ }^{3 \mathrm{~h}}$ and $\mathbf{1 4} \mathbf{i}^{\prime 3 \mathrm{~b}, 3 \mathrm{c}}$ were found to be the dipeptide containing $(Z)-\alpha, \beta$-didehydroamino acid residue according to the reported ${ }^{1} \mathrm{H}$ NMR data.

As mentioned above, the reaction of compound $\mathbf{3 f}$ derived from glycine with a variety of aldehydes $\mathbf{4}$ afforded various kinds of cis-4,5-oxazolidinone derivatives 2 , which were readily transformed to the corresponding $\alpha, \beta$-didehydroamino acid derivatives with high $\mathrm{E}$ selectivity in good yields under basic conditions. Thus, $3 \mathbf{f}$ was found to be a very useful building block for the preparation of $(E)-\alpha, \beta$-didehydroamino acid. Moreover, $(E)-\alpha, \beta$-didehydroamino acid derivatives thus prepared were satisfactorily isomerized to the corresponding $(Z)-\alpha, \beta$-didehydroamino acid derivatives by using iodine. Eventually, the present method will supply a convenient preparation method for both $(E)$ - and $(Z)-\alpha, \beta$-didehydroamino acid derivatives. In addition, the amide compounds 13 were easily transformed to the corresponding dipeptides 14 containing $(E)$ - $\alpha, \beta$-didehydroamino acid residue under basic conditions.

\section{Experimental}

All of the melting points were determined with a micro melting apparatus (Yanagimoto Seisakusyo) and are uncorrected. The ${ }^{1} \mathrm{HNMR}$, IR, and MS spectra were recorded on JEOL JNM-LA $400 \mathrm{FT}(400 \mathrm{MHz})$ and LA $300 \mathrm{FT}(300 \mathrm{MHz})$ NMR spectrometers, a JASCO FT/IR-230 infrared spectrometer, and a JEOL SX-102A mass spectrometer, respectively. The chemical shifts of the NMR spectra are reported in $\delta$-relative to TMS as an internal standard. All solvents were distilled and stored over a drying agent. Thinlayer chromatography (TLC) and flash-column chromatography 
were performed using Merck's silica gel $60 \mathrm{PF}_{254}$ (Art. 7749) and Cica-Merck's silica gel 60 (No. 9385-5B), respectively. All crystalline products prepared in the present work were colorless.

A General Procedure for Conversion of Oxazolidinones 2a21 to $(\boldsymbol{E})-\boldsymbol{\alpha}, \boldsymbol{\beta}$-Didehydroamino Acid Derivatives 1a-11. To a solution of $\mathbf{2 a}(81 \mathrm{mg}, 0.31 \mathrm{mmol})$ in dry THF $(5 \mathrm{~mL})$ was added LiHMDS $(1.55 \mathrm{mmol})$ in THF at $-78^{\circ} \mathrm{C}$ under $\mathrm{N}_{2}$. The solution was stirred for $5 \mathrm{~min}$ at that temperature and quenched with $1 \mathrm{M}$ $\mathrm{AcOH}$ in THF. The solvent was removed in vacuo to give a residue, which was partitioned between ethyl acetate (EtOAc) and water. The EtOAc solution was washed with brine, dried over $\mathrm{MgSO}_{4}$, and concentrated under reduced pressure. The residue was subjected to preparative TLC $\left(\mathrm{SiO}_{2}\right.$, hexane $\left./ \mathrm{EtOAc}=4 / 1, \mathrm{v} / \mathrm{v}\right)$ to afford methyl (E)-2-(Boc-amino)-2-butenoate (1a) in 85\% (57 mg) and $\mathrm{Z}$ isomer, $\mathbf{1} \mathbf{a}^{\prime}$ in $<13 \%$ yields, respectively. $(E)-\mathbf{1 a}$ : oil; IR (neat): 3457, 3420, 3358, 2979, 2954, 2932, 2394, 2287, 1728, $1651,1511,1439,1365,1254,1200,1163,1116,1051,1013,937$, $888,850,829,800,771,703 \mathrm{~cm}^{-1} ;{ }^{1} \mathrm{HNMR}\left(400 \mathrm{MHz}, \mathrm{CDCl}_{3}\right)$ : $\delta 1.47(\mathrm{~s}, 9 \mathrm{H}), 2.03(\mathrm{~d}, J=11.71 \mathrm{~Hz}, 3 \mathrm{H}), 3.86(\mathrm{~s}, 3 \mathrm{H}), 6.57$ (br, 1H), 6.78 (br, 1H). HRMS $\left(\mathrm{FAB}^{+}\right)\left(\mathrm{M}^{+}+1\right)$ : Found: $m / z$ 216.1241. Calcd for $\mathrm{C}_{10} \mathrm{H}_{18} \mathrm{NO}_{4}$ : 216.1236 When the $\beta$-methyl protons were irradiated, $9.0 \%$ and $1.9 \%$ NOE were observed for the olefinic proton and the protons of the methyl ester, respectively. (Z)-1a': mp 72.5-73.5 ${ }^{\circ} \mathrm{C}$ (EtOAc-hexane) (Lit. mp 69-71 $\left.{ }^{\circ} \mathrm{C}\right) .^{8 \mathrm{a}}$ IR (KBr): 3330, 3168, 3007, 2978, 2952, 1722, 1642, 1608, 1586, $1545,1468,1455,1436,1366,1329,1307,1246,1220,1161$, $1085,1048,1026,985,937,910,850,793,756,698 \mathrm{~cm}^{-1}$; ${ }^{1} \mathrm{HNMR}\left(400 \mathrm{MHz}, \mathrm{CDCl}_{3}\right): \delta 1.47(\mathrm{~s}, 9 \mathrm{H}), 1.81(\mathrm{~d}, J=7.32 \mathrm{~Hz}$, $3 \mathrm{H}), 3.77$ (s, 3H), 5.86-6.10 (br, 1H), $6.68(\mathrm{q}, J=7.32 \mathrm{~Hz}, 1 \mathrm{H})$. Found: $\mathrm{C}, 55.60 ; \mathrm{H}, 8.02 ; \mathrm{N}, 6.51 \%$. Calcd for $\mathrm{C}_{10} \mathrm{H}_{17} \mathrm{NO}_{4}$ : C, 55.80; H, 7.96; N, 6.51\%.

The physical and spectral data of compounds $\mathbf{1 b}-\mathbf{1 l}$ and $\mathbf{1} \mathbf{b}^{\prime}-\mathbf{1 \mathbf { l } ^ { \prime }}$ are shown in the following.

Methyl 2-(N-Boc-amino)-2-pentenoate (1b and 1b'): (E)-1b: oil; IR (neat): 3457, 3419, 3360, 2977, 2935, 2876, 2287, 1707, $1645,1512,1457,1438,1391,1366,1248,1163,1050,1020,978$, 934, 876, 832, 816, 772, $702 \mathrm{~cm}^{-1} ;{ }^{1} \mathrm{H} \mathrm{NMR}\left(400 \mathrm{MHz}, \mathrm{CDCl}_{3}\right): \delta$ $1.09(\mathrm{t}, J=7.55 \mathrm{~Hz}, 3 \mathrm{H}), 1.47(\mathrm{~s}, 9 \mathrm{H}), 2.54$ (quintet, $J=7.55 \mathrm{~Hz}$, $2 \mathrm{H}), 3.82(\mathrm{~s}, 3 \mathrm{H}), 6.55(\mathrm{br}, 1 \mathrm{H}), 6.88(\mathrm{br}, 1 \mathrm{H})$. HRMS $\left(\mathrm{FAB}^{+}\right)$ $\left(\mathrm{M}^{+}+1\right)$ : Found: $m / z$ 230.1390. Calcd for $\mathrm{C}_{11} \mathrm{H}_{20} \mathrm{NO}_{4}$ : 230.1392 . When the methyl ester protons were irradiated, $4.0 \%$ and $2.6 \%$ NOE were observed for the methylene and the methyl protons of the ethyl group. (Z)-1 $\mathbf{b}^{\prime}$ : oil (Lit. oil); ${ }^{8 \mathrm{a}}$ IR (neat): 3340, 3120, 2976, 2936, 2877, 1709, 1657, 1496, 1457, 1438, 1391, 1366, 1310, 1247, 1160, 1110, 1049, 1020, 988, 917, 884, 848, 799, 776, 734, $692 \mathrm{~cm}^{-1} ;{ }^{1} \mathrm{HNMR}\left(400 \mathrm{MHz}, \mathrm{CDCl}_{3}\right): \delta 1.06(\mathrm{t}, J=7.56 \mathrm{~Hz}$, $3 \mathrm{H}), 1.46(\mathrm{~s}, 9 \mathrm{H}), 2.24$ (quintet, $J=7.56 \mathrm{~Hz}, 2 \mathrm{H}), 3.77(\mathrm{~s}, 3 \mathrm{H})$, $6.02(\mathrm{br}, 1 \mathrm{H}), 6.53(\mathrm{t}, J=7.56 \mathrm{~Hz}, 1 \mathrm{H})$. HRMS $\left(\mathrm{FAB}^{+}\right)\left(\mathrm{M}^{+}+1\right)$ : Found: $m / z$ 230.1386. Calcd for $\mathrm{C}_{11} \mathrm{H}_{20} \mathrm{NO}_{4}$ : 230.1392 .

Methyl ( $\boldsymbol{N}$-Boc)- $\boldsymbol{\alpha}, \boldsymbol{\beta}$-didehydroleucinate (1c and $\left.1 \mathrm{c}^{\prime}\right)$ : $(E)$ 1c: oil IR (neat): 3419, 3357, 2969, 2934, 2870, 1729, 1708, 1644, $1513,1466,1438,1366,1334,1249,1161,1046,1024,855$, $772 \mathrm{~cm}^{-1} ;{ }^{1} \mathrm{HNMR}\left(400 \mathrm{MHz}, \mathrm{CDCl}_{3}\right): \delta 1.05(\mathrm{~d}, J=6.59 \mathrm{~Hz}$, $6 \mathrm{H}), 1.47$ (s, 9H), 3.22-3.31 (m, 1H), 3.82 (s, 3H), 6.37-6.50 (br, $2 \mathrm{H})$. HRMS $\left(\mathrm{FAB}^{+}\right)\left(\mathrm{M}^{+}+1\right)$ : Found: $m / z, 244.1555$. Calcd for $\mathrm{C}_{12} \mathrm{H}_{22} \mathrm{NO}_{4}$ : 244.1549. (Z)-1 $\mathbf{c}^{\prime}$ : oil (Lit. mp 74-75 $\left.{ }^{\circ} \mathrm{C}\right) ;^{8 \mathrm{a}}$ IR (neat): 3340, 3119, 2964, 2933, 2871, 1710, 1657, 1495, 1438, 1366, $1315,1258,1161,1119,1049,1028,998,958,942,915,890,843$, 796, 778, 765, $742 \mathrm{~cm}^{-1}$; ${ }^{1} \mathrm{HNMR}\left(300 \mathrm{MHz}, \mathrm{CDCl}_{3}\right): \delta 1.05$ $(\mathrm{d}, J=7.87 \mathrm{~Hz}, 6 \mathrm{H}), 1.46(\mathrm{~s}, 9 \mathrm{H}), 2.63-2.79(\mathrm{~m}, 1 \mathrm{H}), 3.77(\mathrm{~s}$, $3 \mathrm{H}), 5.92(\mathrm{br}, 1 \mathrm{H}), 6.38(\mathrm{~d}, J=10.09 \mathrm{~Hz}, 1 \mathrm{H}) . \mathrm{HRMS}\left(\mathrm{FAB}^{+}\right)$ $\left(\mathrm{M}^{+}+1\right)$ : Found: $m / z$ 244.1559. Calcd for $\mathrm{C}_{12} \mathrm{H}_{22} \mathrm{NO}_{4}$ : 244.1549.
Methyl 2-(N-Boc-amino)-5-phenyl-2-pentenoate (1d and 1d'): (E)-1d: oil; IR (neat): 3417, 3358, 3086, 3061, 3027, 2979, 2952, 2931, 2860, 2287, 1947, 1727, 1707, 1646, 1604, $1510,1454,1438,1391,1367,1243,1200,1162,1094,1048$, 1026, 897, 868, 836, 770, 750, $700 \mathrm{~cm}^{-1}$; ${ }^{1} \mathrm{HNMR}(400 \mathrm{MHz}$, $\left.\mathrm{CDCl}_{3}\right): \delta 1.47(\mathrm{~s}, 9 \mathrm{H}), 2.76-2.81(\mathrm{~m}, 2 \mathrm{H}), 2.81-2.88(\mathrm{~m}, 2 \mathrm{H})$, 3.79 (s, 3H), 6.58 (br, 1H), 6.76 (br, 1H), 7.19-7.28 (m, 5H). HRMS $\left(\mathrm{FAB}^{+}\right)\left(\mathrm{M}^{+}+1\right)$ : Found: $m / z$ 306.1709. Calcd for $\mathrm{C}_{17} \mathrm{H}_{24} \mathrm{NO}_{4}$ : 306.1705 . When the methyl protons of the ester group were irradiated, $1.3 \%$ and $1.8 \%$ NOE were observed for the $\gamma, \delta$-methylene protons and the phenyl protons, respectively. (Z)1d'; oil (Lit. mp $48^{\circ} \mathrm{C}$ ). ${ }^{14}$ IR (neat): 3339, 3086, 3062, 3027, 2978, 2952, 2931, 1725, 1657, 1604, 1496, 1454, 1437, 1391, 1367, $1269,1246,1165,1085,1049,1031,1003,912,882,845,798$, $775,749,700 \mathrm{~cm}^{-1} ;{ }^{1} \mathrm{HNMR}\left(400 \mathrm{MHz}, \mathrm{CDCl}_{3}\right): \delta 1.46(\mathrm{~s}, 9 \mathrm{H})$, $2.47(\mathrm{q}, J=7.80 \mathrm{~Hz}, 2 \mathrm{H}), 2.77(\mathrm{t}, J=7.80 \mathrm{~Hz}, 2 \mathrm{H}), 3.76(\mathrm{~s}, 3 \mathrm{H})$, 5.86 (br s, $1 \mathrm{H}), 6.58(\mathrm{t}, J=7.80 \mathrm{~Hz}, 1 \mathrm{H}), 7.20-7.34(\mathrm{~m}, 5 \mathrm{H})$. HRMS $\left(\mathrm{FAB}^{+}\right)\left(\mathrm{M}^{+}+1\right)$ : Found: $m / z$ 306.1702. Calcd for $\mathrm{C}_{17} \mathrm{H}_{24} \mathrm{NO}_{4}$ : 306.1705 .

Methyl $\alpha$-(N-Boc)- $\beta$ - $\left(N^{\prime}-Z\right)-\alpha, \beta$-didehydrotryptophanate (1e and 1e'): (E)-1e; mp 113.0-113.5 ${ }^{\circ} \mathrm{C}$ (EtOAc-hexane). IR (KBr): 3898, 3851, 3733, 3708, 3687, 3674, 3647, 3627, 3415, 2954, 2357, 1745, 1695, 1507, 1457, 1441, 1405, 1360, 1322, 1280, 1238, 1161, 1088, 1043, 910, 756, $704 \mathrm{~cm}^{-1}$; ${ }^{1} \mathrm{HNMR}$ $\left(400 \mathrm{MHz}, \mathrm{CDCl}_{3}\right): \delta 1.52(\mathrm{~s}, 9 \mathrm{H}), 3.70(\mathrm{~s}, 3 \mathrm{H}), 5.47(\mathrm{~s}, 2 \mathrm{H}), 7.25$ $7.41(\mathrm{~m}, 6 \mathrm{H}), 7.48(\mathrm{~d}, J=7.81 \mathrm{~Hz}, 2 \mathrm{H}), 7.59(\mathrm{~d}, J=8.05 \mathrm{~Hz}, 1 \mathrm{H})$, $7.74(\mathrm{~s}, 1 \mathrm{H}), 8.07(\mathrm{~s}, 1 \mathrm{H}), 8.17(\mathrm{~d}, J=8.07 \mathrm{~Hz}, 1 \mathrm{H})$. HRMS $\left(\mathrm{FAB}^{+}\right)\left(\mathrm{M}^{+}+1\right)$ : Found: $m / z$ 451.1875. Calcd for $\mathrm{C}_{25} \mathrm{H}_{27} \mathrm{~N}_{2} \mathrm{O}_{6}$ : 451.1869. When the $\mathrm{N}-\mathrm{H}$ proton of the carbamate was irradiated, 2.8\% NOE was observed for the olefinic proton. (Z)-1 $\mathbf{e}^{\prime}: \mathrm{mp}$ 107.5-108.5 ${ }^{\circ} \mathrm{C}$ (EtOAc-hexane). IR (KBr): 3340, 2978, 2953, $2934,1714,1660,1495,1455,1437,1391,1382,1367,1350$, 1275, 1244, 1165, 1097, 1047, 1008, 949, 899, 835, 797, 774, 764, 728, $695 \mathrm{~cm}^{-1}$; ${ }^{1} \mathrm{HNMR}\left(400 \mathrm{MHz}, \mathrm{CDCl}_{3}\right): \delta 1.56(\mathrm{~s}, 9 \mathrm{H}), 3.87$ (s, 3H), $5.46(\mathrm{~s}, 2 \mathrm{H}), 6.22(\mathrm{br}, 1 \mathrm{H}), 7.31-7.49(\mathrm{~m}, 7 \mathrm{H}), 7.59$ (s, $1 \mathrm{H}), 7.72(\mathrm{~d}, J=7.07 \mathrm{~Hz}, 1 \mathrm{H}), 7.92(\mathrm{~s}, 1 \mathrm{H}), 8.20(\mathrm{~d}, J=7.79 \mathrm{~Hz}$, $1 \mathrm{H})$. Found: $\mathrm{C}, 66.60 ; \mathrm{H}, 5.82 ; \mathrm{N}, 6.26 \%$. Calcd for $\mathrm{C}_{25} \mathrm{H}_{26} \mathrm{~N}_{2} \mathrm{O}_{6}$; C, 66.65; H, 5.82; N, 6.22\%.

Methyl $\alpha$-( $N$-Boc)- $\beta$ - $\left(N^{\prime}\right.$-Boc $)-\alpha, \beta$-didehydrotryptophanate (1f and 1f'): (E)-1f: $\mathrm{mp} 130.5-131.0^{\circ} \mathrm{C}$ (EtOAc-hexane). IR (KBr): 3854, 3735, 3650, 3452, 3188, 2980, 2359, 1727, 1712, $1629,1519,1455,1435,1398,1339,1309,1257,1237,1212$, $1148,1093,1045,1028,986,922,849,765,741,725 \mathrm{~cm}^{-1}$; ${ }^{1} \mathrm{HNMR}\left(400 \mathrm{MHz}, \mathrm{CDCl}_{3}\right): \delta 1.52(\mathrm{~s}, 9 \mathrm{H}), 1.68(\mathrm{~s}, 9 \mathrm{H}), 3.75(\mathrm{~s}$, $3 \mathrm{H}), 6.85(\mathrm{~s}, 1 \mathrm{H}), 7.25-7.32(\mathrm{~m}, 2 \mathrm{H}), 7.57(\mathrm{~d}, J=7.56 \mathrm{~Hz}, 1 \mathrm{H})$, $7.76(\mathrm{~s}, 1 \mathrm{H}), 8.05(\mathrm{~s}, 1 \mathrm{H}), 8.13(\mathrm{~d}, J=8.05 \mathrm{~Hz}, 1 \mathrm{H})$. Found: $\mathrm{C}$, 63.09; H, 6.85; N, 6.67\%. Calcd for $\mathrm{C}_{22} \mathrm{H}_{28} \mathrm{~N}_{2} \mathrm{O}_{6}: \mathrm{C}, 63.44 ; \mathrm{H}$, $6.78 ; \mathrm{N}, 6.73 \%$. When the olefinic proton was irradiated, $1.3 \%$ and $1.0 \%$ NOE were observed for the $t$-butyl protons of the Boc group and the $\mathrm{N}-\mathrm{H}$ proton of the carbamate, respectively. (Z)-1f': $\mathrm{mp}$ 145.0-146.0 ${ }^{\circ} \mathrm{C}$ (EtOAc-hexane). IR (KBr): 3324, 2978, 1734, $1641,1584,1545,1482,1454,1435,1370,1330,1308,1250$, 1153, 1086, 1049, 1019,987,935,858,839,794, $741 \mathrm{~cm}^{-1}$; ${ }^{1} \mathrm{HNMR}\left(400 \mathrm{MHz}, \mathrm{CDCl}_{3}\right): \delta 1.45(\mathrm{~s}, 9 \mathrm{H}), 1.68(\mathrm{~s}, 9 \mathrm{H}), 3.64(\mathrm{~s}$, $3 \mathrm{H}), 6.17(\mathrm{~s}, 1 \mathrm{H}), 7.29-7.36(\mathrm{~m}, 2 \mathrm{H}), 7.59(\mathrm{~s}, 1 \mathrm{H}), 7.70(\mathrm{~d}$, $J=7.83 \mathrm{~Hz}, 1 \mathrm{H}), 7.94(\mathrm{~s}, 1 \mathrm{H}), 8.15(\mathrm{~d}, J=8.03 \mathrm{~Hz}, 1 \mathrm{H})$. Found: C, 63.40; H, 6.84; N, 6.75\%. Calcd for $\mathrm{C}_{22} \mathrm{H}_{28} \mathrm{~N}_{2} \mathrm{O}_{6}: \mathrm{C}, 63.44 ; \mathrm{H}$, $6.78 ; \mathrm{N}, 6.73 \%$.

Methyl $\alpha$-( $N$-Boc)- $\beta$ - $\left(N^{\prime}\right.$-Boc)- $\boldsymbol{\alpha}, \boldsymbol{\beta}$-didehydrohistidinate (1g and 1g'): (E)-1g: $\mathrm{mp} 147.5-149.0^{\circ} \mathrm{C}$ (EtOAc-hexane). IR (KBr): 3389, 3137, 3124, 2983, 2949, 2359, 1736, 1656, 1545, 1496, $1478,1435,1390,1374,1352,1308,1276,1256,1225,1159$, 
1118, 1050, 1026, 1011, 986, 965,895,873,837, $770 \mathrm{~cm}^{-1}$; ${ }^{1} \mathrm{HNMR}\left(400 \mathrm{MHz}, \mathrm{CDCl}_{3}\right): \delta 1.49(\mathrm{~s}, 9 \mathrm{H}), 1.62(\mathrm{~s}, 9 \mathrm{H}), 3.84(\mathrm{~s}$, $3 \mathrm{H}), 6.79(\mathrm{~s}, 1 \mathrm{H}), 7.24(\mathrm{~s}, 1 \mathrm{H}), 7.84(\mathrm{~s}, 1 \mathrm{H}), 8.01$ (s, 1H). Found: C, 55.48; H, 6.96; N, 11.31\%. Calcd for $\mathrm{C}_{17} \mathrm{H}_{25} \mathrm{~N}_{3} \mathrm{O}_{6}: \mathrm{C}, 55.57 ; \mathrm{H}$, $6.86 ; \mathrm{N}, 11.44 \%$. When the $\mathrm{N}-\mathrm{H}$ proton of the carbamate was irraditated, $1.8 \% \mathrm{NOE}$ was observed for the olefinic proton. $(Z)$ 1g': mp 120.5-121.5 ${ }^{\circ} \mathrm{C}$ (EtOAc-hexane). IR (KBr): 3300, 3134, 2981, 1755, 1738, 1718, 1649, 1550, 1466, 1393, 1368, 1331, $1257,1234,1214,1156,1063,1013,839,805,770 \mathrm{~cm}^{-1} ;{ }^{1} \mathrm{HNMR}$ $\left(400 \mathrm{MHz}, \mathrm{CDCl}_{3}\right): \delta 1.48(\mathrm{~s}, 9 \mathrm{H}), 1.63(\mathrm{~s}, 9 \mathrm{H}), 3.84(\mathrm{~s}, 3 \mathrm{H}), 6.42$ (s, 1H), 7.37 (s, 1H), 8.09 (s, 1H), $9.04(\mathrm{~s}, 1 \mathrm{H})$. Found: C, 55.59; $\mathrm{H}, 6.98 ; \mathrm{N}, 11.27 \%$. Calcd for $\mathrm{C}_{17} \mathrm{H}_{25} \mathrm{~N}_{3} \mathrm{O}_{6}: \mathrm{C}, 55.57 ; \mathrm{H}, 6.86 ; \mathrm{N}$, $11.44 \%$.

Methyl $\alpha$-( $N$-Boc)- $\varepsilon-\left(N^{\prime}\right.$-Boc- $\left.N^{\prime}-Z\right)-\alpha, \beta$-didehydrolysinate (1h and 1h'): (E)-1h: oil; IR (neat): 3365, 3065, 3033, 2979, 2935, 2401, 2291, 1790, 1738, 1588, 1500, 1479, 1456, 1369, 1342, 1286, 1247, 1208, 1154, 1047, 973, 916, 854, 780, 753, $699 \mathrm{~cm}^{-1} ;{ }^{1} \mathrm{HNMR}\left(400 \mathrm{MHz}, \mathrm{CDCl}_{3}\right): \delta 1.46(\mathrm{~s}, 9 \mathrm{H}), 1.47(\mathrm{~s}$, $9 \mathrm{H}), 1.75(\mathrm{tt}, J=7.51,7.69 \mathrm{~Hz}, 2 \mathrm{H}), 2.52(\mathrm{dt}, J=7.70,7.72 \mathrm{~Hz}$, $2 \mathrm{H}), 3.68(\mathrm{t}, J=7.70 \mathrm{~Hz}, 2 \mathrm{H}), 3.78(\mathrm{~s}, 3 \mathrm{H}), 5.22(\mathrm{~s}, 2 \mathrm{H}), 6.55(\mathrm{br}$, 1H), 6.66 (br, 1H), 7.29-7.40 (m, 5H). HRMS $\left(\mathrm{FAB}^{+}\right)\left(\mathrm{M}^{+}+1\right)$ : Found: $m / z$ 493.2564. Calcd for $\mathrm{C}_{25} \mathrm{H}_{37} \mathrm{~N}_{2} \mathrm{O}_{8}$ : 493.2550. (Z)-1h': oil; IR (neat): 3342, 2979, 1789, 1723, 1497, 1456, 1368, 1287, $1159,1114,1047,915,854,778,698 \mathrm{~cm}^{-1}$; ${ }^{1} \mathrm{H}$ NMR (400 MHz, $\left.\mathrm{CDCl}_{3}\right): \delta 1.45(\mathrm{~s}, 9 \mathrm{H}), 1.47(\mathrm{~s}, 9 \mathrm{H}), 1.75$ (quintet, $J=7.52 \mathrm{~Hz}$, $2 \mathrm{H}), 2.22(\mathrm{dt}, J=7.15,7.52 \mathrm{~Hz}, 2 \mathrm{H}), 3.66(\mathrm{t}, J=7.52 \mathrm{~Hz}, 2 \mathrm{H})$, 3.75 (s, 3H), 5.22 (s, 2H), 6.12 (br, 1H), 6.49 (t, $J=7.15 \mathrm{~Hz}, 1 \mathrm{H})$, 7.30-7.40 (m, 5H). HRMS $\left(\mathrm{FAB}^{+}\right)\left(\mathrm{M}^{+}+1\right)$ : Found: $m / z$ 493.2545. Calcd for $\mathrm{C}_{25} \mathrm{H}_{37} \mathrm{~N}_{2} \mathrm{O}_{8}: 493.2550$.

Methyl 2-( $N$-Boc-amino)-3-phenylpropenoate (1i and 1i'): (E)-1i: oil; IR (neat): 3333, 2979, 1715, 1636, 1514, 1490, 1437, 1368, 1244, 1157, 1057, 1026, 984, 919, 857, 833, 754, $698 \mathrm{~cm}^{-1}$; ${ }^{1} \mathrm{HNMR}\left(300 \mathrm{MHz}, \mathrm{CDCl}_{3}\right): \delta 1.50(\mathrm{~s}, 9 \mathrm{H}), 3.63(\mathrm{~s}, 3 \mathrm{H}), 6.68-$ 6.74 (br, 1H), 7.21-7.32 (m, 5H), 7.40-7.60 (br, 1H). EI-MS $m / z$ $277\left(\mathrm{M}^{+} ; 6.12 \%\right)$. When the $\mathrm{N}-\mathrm{H}$ proton was irradiated, $3.1 \%$ and $1.0 \%$ NOE were observed for the olefinic proton and the protons of the methyl ester. $(Z)-1 \mathbf{i}: \mathrm{mp} 76.0-77.0^{\circ} \mathrm{C}$ (hexane) (Lit. mp 81$82^{\circ} \mathrm{C}^{8 \mathrm{a}} 77-79^{\circ} \mathrm{C}^{8 \mathrm{~b}}$ ); IR (KBr): 3327, 2990, 2946, 1721, 1700, $1644,1488,1445,1369,1287,1242,1206,1163,1143,1079$, 1063, 990, 841, 761, $690 \mathrm{~cm}^{-1} ;{ }^{1} \mathrm{HNMR}\left(300 \mathrm{MHz}, \mathrm{CDCl}_{3}\right): \delta$ 1.40 (s, 9H), $3.86(\mathrm{~s}, 3 \mathrm{H}), 6.06-6.24$ (br, 1H), 7.25 (s, 1H), 7.317.40 (m, 3H), 7.52-7.55 (m, 2H). Found: C, 64.80; H, 6.97; N, $5.09 \%$. Calcd for $\mathrm{C}_{15} \mathrm{H}_{19} \mathrm{NO}_{4}: \mathrm{C}, 64.97 ; \mathrm{H}, 6.91 ; \mathrm{N}, 5.05 \%$.

Methyl 3-(p-Bromophenyl)-2-( $N$-Boc-amino)propenoate (1j and $\left.\mathbf{1 j}^{\prime}\right)$ : (E)-1j: oil; IR (neat): 3333, 2979, 1725, 1715, 1636, 1514, 1490, 1437, 1368, 1244, 1157, 1057, 1026, 984, 919, 857, $833,754,698 \mathrm{~cm}^{-1} ;{ }^{1} \mathrm{HNMR}\left(300 \mathrm{MHz}, \mathrm{CDCl}_{3}\right): \delta 1.50(\mathrm{~s}, 9 \mathrm{H})$, 3.65 (s, 3H), 6.72-6.82 (br, 1H), 7.10 (d, $J=8.44 \mathrm{~Hz}, 2 \mathrm{H}), 7.41$ $(\mathrm{d}, J=8.44 \mathrm{~Hz}, 2 \mathrm{H}), 7.45-7.55(\mathrm{br}, 1 \mathrm{H})$. EI-MS $m / z 355\left(\mathrm{M}^{+}\right.$; $10.02 \%)$. When the $\mathrm{N}-\mathrm{H}$ proton of the carbamate was irradiated, $3.5 \%$ and $1 \%$ NOE were observed for the olefinic proton and both ortho protons of the aromatic ring, respectively. (Z)-1j': mp 121.0-122. $0^{\circ} \mathrm{C}$ (EtOAc-hexane). IR (KBr): 3327, 2990, 2946, $1721,1700,1644,1488,1445,1369,1287,1242,1206,1163$, 1143, 1063, 990, 841, 761, $690 \mathrm{~cm}^{-1}$; ${ }^{1} \mathrm{HNMR}\left(300 \mathrm{MHz}, \mathrm{CDCl}_{3}\right)$ : $\delta 1.40(\mathrm{~s}, 9 \mathrm{H}), 3.86(\mathrm{~s}, 3 \mathrm{H}), 6.20-6.30(\mathrm{br}, 1 \mathrm{H}), 7.19(\mathrm{~s}, 1 \mathrm{H}), 7.39$ (d, $J=8.62 \mathrm{~Hz}, 2 \mathrm{H}), 7.48$ (d, $J=8.62 \mathrm{~Hz}, 2 \mathrm{H})$. Found: C, 50.47; $\mathrm{H}, 5.17 ; \mathrm{N}, 3.85 \%$. Calcd for $\mathrm{C}_{15} \mathrm{H}_{18} \mathrm{NO}_{4} \mathrm{Br}$ : C, 50.58; H, 5.09; N, $3.93 \%$.

Methyl 2-(N-Boc-amino)-3-(p-tolyl)propenoate (1k and $\mathbf{1 k}$ '): (E)-1k: oil; IR (neat): 3355, 2979, 1725, 1708, 1637, 1607, 1507, $1485,1438,1368,1248,1159,1050,1023,984,845,815$,
$775 \mathrm{~cm}^{-1} ;{ }^{1} \mathrm{HNMR}\left(300 \mathrm{MHz}, \mathrm{CDCl}_{3}\right): \delta 1.49(\mathrm{~s}, 9 \mathrm{H}), 2.33(\mathrm{~s}$, $3 \mathrm{H}), 3.66$ (s, 3H), 6.62-6.70 (br, $1 \mathrm{H}), 7.09$ (d, $J=8.34 \mathrm{~Hz}, 2 \mathrm{H})$, $7.14(\mathrm{~d}, J=8.34 \mathrm{~Hz}, 2 \mathrm{H}), 7.38-7.48(\mathrm{br}, 1 \mathrm{H})$. EI-MS $m / z, 291$ $\left(\mathrm{M}^{+} ; 15.51 \%\right)$. When the $\mathrm{N}-\mathrm{H}$ proton of the carbamate was irradiated, $2.8 \%$ NOE was observed for the olefinic proton. (Z)1k': mp 88.0-89.0 ${ }^{\circ} \mathrm{C}$ (hexane) (Lit. 87-90 $\left.{ }^{\circ} \mathrm{C}\right) .{ }^{8 \mathrm{c}} \mathrm{IR}(\mathrm{KBr}): 3339$, 2971, 1708, 1631, 1605, 1488, 1444, 1369, 1342, 1272, 1172, 1136, 1049, 1024, 987, 921, 820, 795, $775 \mathrm{~cm}^{-1}$; ${ }^{1} \mathrm{HNMR}$ $\left(300 \mathrm{MHz}, \mathrm{CDCl}_{3}\right): \delta 1.41(\mathrm{~s}, 9 \mathrm{H}), 2.36(\mathrm{~s}, 3 \mathrm{H}), 3.85(\mathrm{~s}, 3 \mathrm{H})$, 6.07-6.17 (br, $1 \mathrm{H}), 7.17$ (d, $J=8.07 \mathrm{~Hz}, 2 \mathrm{H}), 7.25-7.48$ (br, $1 \mathrm{H})$, $7.44(\mathrm{~d}, J=8.07 \mathrm{~Hz}, 2 \mathrm{H})$. Found: C, 65.66; H, 7.48; N, $4.71 \%$. Calcd for $\mathrm{C}_{16} \mathrm{H}_{21} \mathrm{NO}_{4}$ : C, 65.96; $\mathrm{H}, 7.27 ; \mathrm{N}, 4.81 \%$.

Methyl 2-(N-Boc-amino)-3-(p-methoxyphenyl)propenoate (11 and 11'): (E)-11: oil; IR (neat): 3338, 2979, 1723, 1637, $1607,1512,1438,1368,1302,1248,1161,1030,985,919$, $824 \mathrm{~cm}^{-1} ;{ }^{1} \mathrm{HNMR}\left(300 \mathrm{MHz}, \mathrm{CDCl}_{3}\right): \delta 1.49(\mathrm{~s}, 9 \mathrm{H}), 3.68(\mathrm{~s}$, $3 \mathrm{H}), 3.81$ (s, 3H), 6.60-6.70 (br, $1 \mathrm{H}), 6.83$ (d, $J=8.90 \mathrm{~Hz}, 2 \mathrm{H})$, $7.21(\mathrm{~d}, J=8.90 \mathrm{~Hz}, 2 \mathrm{H}), 7.34-7.44(\mathrm{br}, 1 \mathrm{H})$. EI-MS $m / z, 307$ $\left(\mathrm{M}^{+} ; 15.51 \%\right)$. When the $\mathrm{N}-\mathrm{H}$ proton of the carbamate was irradiated, $4.1 \%$ NOE was observed for the olefinic proton. $(Z)-1 l^{\prime}$ : mp 111.0-112.0 ${ }^{\circ} \mathrm{C}$ (EtOAc-hexane). IR (KBr): 3338, 2979, 1703, $1638,1604,1515,1442,1360,1321,1259,1181,1145,1055$, 1030, 992, 826, $779 \mathrm{~cm}^{-1}$; ${ }^{1} \mathrm{HNMR}\left(400 \mathrm{MHz}, \mathrm{CDCl}_{3}\right): \delta 1.49(\mathrm{~s}$, $9 \mathrm{H}), 3.79(\mathrm{~s}, 3 \mathrm{H}), 3.80(\mathrm{~s}, 3 \mathrm{H}), 6.69-6.77(\mathrm{br}, 1 \mathrm{H}), 6.82(\mathrm{~d}$, $J=8.80 \mathrm{~Hz}, 2 \mathrm{H}), 7.26-7.40(\mathrm{br}, 1 \mathrm{H}), 7.51(\mathrm{~d}, J=8.80 \mathrm{~Hz}, 2 \mathrm{H})$. Found: $\mathrm{C}, 62.32 ; \mathrm{H}, 7.03 ; \mathrm{N}, 4.47 \%$. Calcd for $\mathrm{C}_{16} \mathrm{H}_{21} \mathrm{NO}_{5}$ : C, 62.53; H, 6.89; N, $4.56 \%$.

A General Procedure for the Preparation of cis-4,5-Oxazolidin-2-one Derivatives (2a-2l). To a solution of $\mathbf{3 f}(93 \mathrm{mg}$, $0.30 \mathrm{mmol})$ in dry THF $(1.5 \mathrm{~mL})$ was added 1.1 molar equivalents of LDA at $-78^{\circ} \mathrm{C}$ under $\mathrm{N}_{2}$. After the solution was stirred at that temperature for $1 \mathrm{~h}$, a solution of benzaldehyde (4i) $(35 \mathrm{mg}$, $0.33 \mathrm{mmol})$ in THF $(1.5 \mathrm{~mL})$ was added dropwise and the solution was kept at $-78^{\circ} \mathrm{C}$ for $1 \mathrm{~h}$. The reaction was then quenched with $1 \mathrm{M} \mathrm{AcOH}$ in $\mathrm{MeOH}$ and the solvent was concentrated under reduced pressure. The residue was partitioned between EtOAc and water. The aqueous layer was extracted with EtOAc and the combined extracts were washed with brine, dried over $\mathrm{MgSO}_{4}$, and evaporated in vacuo. The residual oil was subjected to preparative TLC $\left(\mathrm{SiO}_{2}\right.$, hexane/EtOAc $\left.=5 / 1, \mathrm{v} / \mathrm{v}\right)$ to give 3-Boc-4-(methoxycarbonyl)-5-phenyl-cis-4,5-oxazolidin-2-one (2i) in $63 \%$ yield (203 mg). Mp 129.0-130.0 ${ }^{\circ} \mathrm{C}$ (EtOAc-hexane) (Lit, mp 114$\left.115^{\circ} \mathrm{C}\right) ;{ }^{9 \mathrm{a}} \mathrm{IR}(\mathrm{KBr}): 2976,1803,1750,1723,1457,1441,1388$, 1365, 1312, 1260, 1218, 1189, 1167, 1088, 1050, 1006, 927, 861, 837, 786, 766, $699 \mathrm{~cm}^{-1} ;{ }^{1} \mathrm{HNMR}\left(300 \mathrm{MHz}, \mathrm{CDCl}_{3}\right): \delta 1.51(\mathrm{~s}$, $9 \mathrm{H}), 3.25(\mathrm{~s}, 3 \mathrm{H}), 4.95(\mathrm{~d}, J=8.90 \mathrm{~Hz}, 1 \mathrm{H}), 5.71(\mathrm{~d}, J=8.90 \mathrm{~Hz}$, $1 \mathrm{H}), 7.28-7.40(\mathrm{~m}, 2 \mathrm{H}), 7.50-7.55(\mathrm{~m}, 3 \mathrm{H})$. Found: C, 59.59; H, $6.19 ; \mathrm{N}, 4.31 \%$. Calcd for $\mathrm{C}_{16} \mathrm{H}_{19} \mathrm{NO}_{6}: \mathrm{C}, 59.81 ; \mathrm{H}, 5.96 ; \mathrm{N}$, $4.36 \%$. When the proton at $\mathrm{C}-4$ was irradiated, $12.0 \%$ and $2.4 \%$ NOE were observed for the proton at C-5 and both ortho protons of the aromatic ring. When the proton at C-5 was irradiated, $15.1 \%$ and $11.0 \%$ NOE were observed for the proton at C-4 and both ortho protons of the aromatic ring.

The physical and spectral data of compounds $\mathbf{2 a}-\mathbf{2 h}$ and $\mathbf{2} \mathbf{j}-\mathbf{2 l}$ are shown in the following.

3-Boc-4-methoxycarbonyl-5-methyl-cis-4,5-oxazolidin-2-one

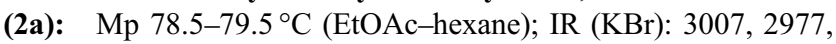
2954, 2939, 2884, 1826, 1759, 1724, 1701, 1587, 1463, 1442, $1396,1371,1327,1299,1281,1260,1220,1185,1160,1120$, $1074,1025 \mathrm{~cm}^{-1}$; ${ }^{1} \mathrm{HNMR}\left(400 \mathrm{MHz}, \mathrm{CDCl}_{3}\right): \delta 1.38(\mathrm{~d}, J=6.34$ $\mathrm{Hz}, 3 \mathrm{H}), 1.51$ (s, 9H), $3.83(\mathrm{~s}, 3 \mathrm{H}), 4.72(\mathrm{~d}, J=8.78 \mathrm{~Hz}, 1 \mathrm{H}), 4.78$ $(\mathrm{dq}, J=6.34,8.78 \mathrm{~Hz}, 1 \mathrm{H})$. Found: C, $50.88 ; \mathrm{H}, 6.67 ; \mathrm{N}, 5.46 \%$. 
Calcd for $\mathrm{C}_{11} \mathrm{H}_{17} \mathrm{NO}_{6}$ : C, 50.96; $\mathrm{H}, 6.61 ; 5.40 \%$. When the protons of the methyl ester were irradiated, $1.0 \%, 1.82 \%$, and $1.34 \% \mathrm{NOE}$ were observed for the proton at C-4, both $t$-butyl protons of the Boc group and the methyl protons at $\mathrm{C}-5$, respectively.

3-Boc-5-ethyl-4-methoxycarbonyl-cis-4,5-oxazolidin-2-one (2b): $\mathrm{Mp} 60.0-63.0^{\circ} \mathrm{C}$ (EtOAc-hexane); IR (KBr): 3007, 2977, 2954, 2939, 2884, 1826, 1759, 1724, 1701, 1463, 1442, 1396, $1371,1327,1299,1281,1260,1220,1185,1160,1120,1074$, $925 \mathrm{~cm}^{-1} ;{ }^{1} \mathrm{HNMR}\left(300 \mathrm{MHz}, \mathrm{CDCl}_{3}\right): \delta 1.08(\mathrm{t}, J=7.34 \mathrm{~Hz}$, $3 \mathrm{H}), 1.51(\mathrm{~s}, 9 \mathrm{H}), 1.59-1.68(\mathrm{~m}, 2 \mathrm{H}), 3.81(\mathrm{~s}, 3 \mathrm{H}), 4.51(\mathrm{dt}$, $J=4.56,8.44 \mathrm{~Hz}, 1 \mathrm{H}), 4.72(\mathrm{~d}, J=8.44 \mathrm{~Hz}, 1 \mathrm{H})$. Found: C, $52.62 ; \mathrm{H}, 7.09 ; \mathrm{N}, 5.07 \%$. Calcd for $\mathrm{C}_{12} \mathrm{H}_{19} \mathrm{NO}_{6}: \mathrm{C}, 52.74 ; \mathrm{H}, 7.01$; $\mathrm{N}, 5.13 \%$.

3-Boc-4-methoxycarbonyl-5-isopropyl-cis-4,5-oxazolidin-2one (2c): $\quad \mathrm{Mp} 82.0-84.0^{\circ} \mathrm{C}$ (EtOAc-hexane); IR (KBr) 2982, $1824,1787,1747,1371,1361,1339,1331,1248,1213,1188$, 1161, 1125, 1096, 1050, 1026, $988 \mathrm{~cm}^{-1}$; ${ }^{1} \mathrm{H} \mathrm{NMR}(300 \mathrm{MHz}$, $\left.\mathrm{CDCl}_{3}\right): \delta 1.02(\mathrm{~d}, J=6.40 \mathrm{~Hz}, 3 \mathrm{H}), 1.08(\mathrm{~d}, J=6.40, \mathrm{~Hz}, 3 \mathrm{H})$, $1.51(\mathrm{~s}, 9 \mathrm{H}), 1.72-1.79(\mathrm{~m}, 2 \mathrm{H}), 3.81(\mathrm{~s}, 3 \mathrm{H}), 4.15(\mathrm{dd}, J=7.52$, $10.09 \mathrm{~Hz}, 1 \mathrm{H}), 4.72(\mathrm{~d}, J=7.52 \mathrm{~Hz}, 1 \mathrm{H})$. Found: C, 54.04; H, $7.35 ; \mathrm{N}, 4.77 \%$. Calcd for $\mathrm{C}_{13} \mathrm{H}_{21} \mathrm{NO}_{6}: \mathrm{C}, 54.35 ; \mathrm{H}, 7.37 ; \mathrm{N}$, $4.88 \%$.

3-Boc-4-methoxycarbonyl-5-(2-phenylethyl)-cis-4,5-oxazolidin-2-one (2d): $\mathrm{Mp} 123.0-125.0^{\circ} \mathrm{C}$ (EtOAc-hexane); IR (KBr): 2979, 1810, 1746, 1702, 1383, 1371, 1352, 1207, 1166, 1060, 1017, 977, 785, 775, $770 \mathrm{~cm}^{-1} ;{ }^{1} \mathrm{HNMR}\left(300 \mathrm{MHz}, \mathrm{CDCl}_{3}\right): \delta$ $1.50(\mathrm{~s}, 9 \mathrm{H}), 1.84-1.90(\mathrm{~m}, 2 \mathrm{H}), 2.72-2.91(\mathrm{~m}, 2 \mathrm{H}), 3.80(\mathrm{~s}, 3 \mathrm{H})$, $4.53(\mathrm{dt}, J=4.95,8.44 \mathrm{~Hz}, 1 \mathrm{H}), 4.68(\mathrm{~d}, J=8.44 \mathrm{~Hz}, 1 \mathrm{H}), 7.17-$ 7.31 (m, 1H). Found: C, 61.82; H, 6.77; N, 4.01\%. Calcd for $\mathrm{C}_{18} \mathrm{H}_{23} \mathrm{NO}_{6}: \mathrm{C}, 61.88 ; \mathrm{H}, 6.64 ; \mathrm{N}, 4.01 \%$.

3-Boc-4-methoxycarbonyl-5-[( $\left.N^{\prime}-\mathrm{Z}\right)-3^{\prime}$-indolyl]-cis-4,5-oxazolidin-2-one (2e): To a solution of $\mathbf{3 f}(93 \mathrm{mg}, 0.30 \mathrm{mmol})$ in dry THF $(5 \mathrm{~mL})$ was added 1.1 molar equivalents of LDA $(0.33 \mathrm{mmol})$ at $-78^{\circ} \mathrm{C}$ under $\mathrm{N}_{2}$. After keeping at $-78^{\circ} \mathrm{C}$ for $1 \mathrm{~h}$, a solution of 4e $(84 \mathrm{mg}, 0.30 \mathrm{mmol})$ in dry THF $(2 \mathrm{~mL})$ was added followed by the addition of $\mathrm{Ti}\left(\mathrm{O}^{i} \mathrm{Pr}\right)_{4}(94 \mathrm{mg}, 0.33 \mathrm{mmol})$. The solution was stirred at $-78^{\circ} \mathrm{C}$ for $4 \mathrm{~h}$ and quenched with $1 \mathrm{M} \mathrm{AcOH}$ in THF. After addition of a small amount of water, the mixture was passed through celite. The THF was removed under reduced pressure to give a residue, which was partitioned between EtOAc and water. The EtOAc solution was washed with brine, dried, and evaporated in vacuo to afford a residue, which was subjected to preparative TLC $\left(\mathrm{SiO}_{2}\right.$, hexane/EtOAc $\left.=3 / 1, \mathrm{v} / \mathrm{v}\right) .98 \mathrm{mg}$ of $2 \mathrm{e}$ was obtained. Mp 173.0-173.5 ${ }^{\circ} \mathrm{C}$ (EtOAc-hexane); IR (KBr): 2979, 1804, 1742, 1609, 1573, 1457, 1407, 1370, 1357, 1315, 1258, $1243,1220,1172,1131,1102,1055,996,756 \mathrm{~cm}^{-1} ;{ }^{1} \mathrm{H}$ NMR $(400$ $\left.\mathrm{MHz}, \mathrm{CDCl}_{3}\right): \delta 1.52(\mathrm{~s}, 9 \mathrm{H}), 3.16(\mathrm{~s}, 3 \mathrm{H}), 5.05(\mathrm{~d}, J=8.78 \mathrm{~Hz}$, $1 \mathrm{H}), 5.43(\mathrm{~d}, J=12.20 \mathrm{~Hz}, 1 \mathrm{H}), 5.47(\mathrm{~d}, J=12.20 \mathrm{~Hz}, 1 \mathrm{H}), 5.95$ $(\mathrm{dd}, J=0.98,8.78 \mathrm{~Hz}, 1 \mathrm{H}), 7.29-7.50(\mathrm{~m}, 8 \mathrm{H}), 7.70(\mathrm{~s}, 1 \mathrm{H}), 8.19$ (d, $J=8.29 \mathrm{~Hz}, 1 \mathrm{H})$. Found: C, 63.08; H, 5.33; N, 5.67\%. Calcd for $\mathrm{C}_{26} \mathrm{H}_{26} \mathrm{~N}_{2} \mathrm{O}_{8}$ : C, 63.15; H, 5.30; H, 5.66\%. When the proton at C-4 was irradiated, $17.5 \%, 4.3 \%$, and $3.3 \%$ NOE were observed for the proton at C-5, 2-position of the indole ring and both the $t$-butyl protons of the Boc group.

3-Boc-4-methoxycarbonyl-5-[( $N^{\prime}$-Boc $)-3^{\prime}$-indolyl $]-c i s-4,5-$ oxazolidin-2-one (2f): $\quad \mathrm{Mp} 152.0-153.0^{\circ} \mathrm{C}$ (EtOAc); IR (KBr): 2981, 2367, 1800, 1742, 1655, 1596, 1559, 1542, 1508, 1458, 1356, 1318, 1258, 1224, 1154, 1131, 1101, 1051, 992, 844, 788, $770,744 \mathrm{~cm}^{-1} ;{ }^{1} \mathrm{HNMR}\left(400 \mathrm{MHz}, \mathrm{CDCl}_{3}\right): \delta 1.52(\mathrm{~s}, 9 \mathrm{H}), 1.56$ $(\mathrm{s}, 9 \mathrm{H}), 3.19(\mathrm{~s}, 3 \mathrm{H}), 5.06(\mathrm{~d}, J=8.78 \mathrm{~Hz}, 1 \mathrm{H}), 5.98(\mathrm{dd}, J=0.98$, $8.78 \mathrm{~Hz}, 1 \mathrm{H}), 7.24-7.27(\mathrm{~m}, 1 \mathrm{H}), 7.33-7.37(\mathrm{~m}, 1 \mathrm{H}), 7.49(\mathrm{~d}$, $J=7.81 \mathrm{~Hz}, 1 \mathrm{H}), 7.65$ (s, 1H), 8.17 (d, $J=8.29 \mathrm{~Hz}, 1 \mathrm{H})$. Found:
C, 59.91; H, 6.25; N, 6.04\%. Calcd for $\mathrm{C}_{23} \mathrm{H}_{28} \mathrm{~N}_{2} \mathrm{O}_{8}$ : C, 59.99; $\mathrm{H}$, $6.13 ; \mathrm{N}, 6.08 \%$. When the proton at $\mathrm{C}-5$ was irradiated, $15.9 \%$ NOE was observed for the proton at C-4.

3-Boc-4-methoxycarbonyl-5-[( $N^{\prime}$-Boc $)-4^{\prime}$-imidazolyl $]-c i s-4,5-$ oxazolidin-2-one (2g): $\mathrm{Mp} 138.0-139.0^{\circ} \mathrm{C}$ (EtOAc); IR (KBr): 3003, 2979, 2935, 1819, 1802, 1750, 1725, 1399, 1368, 1328, 1312, 1276, 1248, 1219, 1190, 1156, 1091, 1069, 1020, 1006, 848, $776 \mathrm{~cm}^{-1}$; ${ }^{1} \mathrm{HNMR}\left(400 \mathrm{MHz}, \mathrm{CDCl}_{3}\right): \delta 1.51(\mathrm{~s}, 9 \mathrm{H}), 1.61(\mathrm{~s}$, $9 \mathrm{H}), 3.60(\mathrm{~s}, 3 \mathrm{H}), 5.01(\mathrm{~d}, J=8.78 \mathrm{~Hz}, 1 \mathrm{H}), 5.70(\mathrm{~d}, J=8.78 \mathrm{~Hz}$, $1 \mathrm{H}), 7.44(\mathrm{~s}, 1 \mathrm{H}), 8.04$ (s, 1H). Found: C, 52.61; H, 6.21; N, $10.04 \%$. Calcd for $\mathrm{C}_{18} \mathrm{H}_{25} \mathrm{~N}_{3} \mathrm{O}_{8}: \mathrm{C}, 52.55 ; \mathrm{H}, 6.13 ; \mathrm{N}, 10.21 \%$.

3-Boc-4-methoxycarbonyl-5-\{3-[( $N^{\prime}$-Boc- $\left.N^{\prime}-Z\right)$ aminopropyl $\left.]\right\}-$ cis-4,5-oxazolidin-2-one (2h): Mp 83.5-84.5 ${ }^{\circ} \mathrm{C}$ (EtOAchexane); IR (KBr): 2979, 1810, 1740, 1654, 1559, 1542, 1508, 1458, 1363, 1339, 1279, 1251, 1164, 1129, 1088, 984, 846, 773, 743, $696 \mathrm{~cm}^{-1}$; ${ }^{1} \mathrm{HNMR}\left(400 \mathrm{MHz}, \mathrm{CDCl}_{3}\right): \delta 1.47(\mathrm{~s}, 9 \mathrm{H}), 1.50$ (s, 9H), 1.50-1.90 (m, 4H), 3.64-3.74 (m, 2H), $3.77(\mathrm{~s}, 3 \mathrm{H}), 4.56$ $(\mathrm{dt}, J=3.84,8.61 \mathrm{~Hz}, 1 \mathrm{H}), 4.66(\mathrm{~d}, J=8.61 \mathrm{~Hz}, 1 \mathrm{H}), 5.21(\mathrm{~s}$, 2H), 7.29-7.39 (m, 5H). Found: C, 58.09; H, 6.79; N, 5.33\%. Calcd for $\mathrm{C}_{26} \mathrm{H}_{36} \mathrm{~N}_{2} \mathrm{O}_{10}$ : C, 58.20; H, 6.76; H, 5.22\%.

3-Boc-5-( $p$-bromophenyl)-4-methoxycarbonyl-cis-4,5-oxazo-

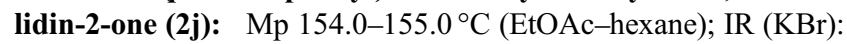
2980, 1810, 1752, 1721, 1361, 1314, 1222, 1160, 1082, 1061, 1006, 846, 787, 774, 766, $694 \mathrm{~cm}^{-1}$; ${ }^{1} \mathrm{HNMR}\left(400 \mathrm{MHz}, \mathrm{CDCl}_{3}\right)$ : $\delta 1.51(\mathrm{~s}, 9 \mathrm{H}), 3.32(\mathrm{~s}, 3 \mathrm{H}), 4.96(\mathrm{~d}, J=8.90 \mathrm{~Hz}, 1 \mathrm{H}), 5.67(\mathrm{~d}$, $J=8.90 \mathrm{~Hz}, 1 \mathrm{H}), 7.20(\mathrm{~d}, J=8.35 \mathrm{~Hz}, 2 \mathrm{H}), 7.53(\mathrm{~d}, J=8.35 \mathrm{~Hz}$, $2 \mathrm{H})$. Found: C, 48.03; H, 4.59; N, 3.42\%. Calcd for $\mathrm{C}_{16} \mathrm{H}_{18} \mathrm{NO}_{6} \mathrm{Br}$ : C, $48.02 ; \mathrm{H}, 4.53 ; \mathrm{N}, 3.50 \%$. When the $\alpha$-proton of the ester group was irradiated, $13.8 \%$ NOE was observed for the $\beta$-proton.

3-Boc-4-methoxycarbonyl-5-(p-tolyl)-cis-4,5-oxazolidin-2-one (2k): $\quad$ Mp 128.0-129.0 ${ }^{\circ} \mathrm{C}$ (EtOAc-hexane); IR (KBr): 2979, $1801,1749,1721,1365,1318,1216,1186,1171,1091,1048$, 1007, 843, 810, 784, $767 \mathrm{~cm}^{-1} ;{ }^{1} \mathrm{H}$ NMR $\left(300 \mathrm{MHz}, \mathrm{CDCl}_{3}\right): \delta$ $1.51(\mathrm{~s}, 9 \mathrm{H}), 2.35(\mathrm{~s}, 3 \mathrm{H}), 3.29(\mathrm{~s}, 3 \mathrm{H}), 4.94(\mathrm{~d}, J=8.99 \mathrm{~Hz}, 1 \mathrm{H})$, $5.67(\mathrm{~d}, J=8.99 \mathrm{~Hz}, 1 \mathrm{H}), 7.18$ (br s, 4H). Found: C, 60.71; H, 6.41; N, 4.16\%. Calcd for $\mathrm{C}_{17} \mathrm{H}_{21} \mathrm{NO}_{6}: \mathrm{C}, 60.89 ; \mathrm{H}, 6.31 ; \mathrm{N}$, $4.18 \%$. When the $\alpha$-proton was irradiated, $13.3 \% \mathrm{NOE}$ was observed for the $\beta$-proton.

3-Boc-4-methoxycarbonyl-5-(p-methoxyphenyl)-cis-4,5-oxazolidin-2-one (2l): $\quad \mathrm{Mp} 140.0-141.00^{\circ} \mathrm{C}$ (EtOAc-hexane); IR (KBr): 2979, 1802, 1747, 1721, 1614, 1518, 1447, 1367, 1314, 1251, 1225, 1177, 1161, 1090, 1052, 1034, 1002, 846, $776 \mathrm{~cm}^{-1}$; ${ }^{1} \mathrm{HNMR}\left(300 \mathrm{MHz}, \mathrm{CDCl}_{3}\right): \delta 1.50(\mathrm{~s}, 9 \mathrm{H}), 3.32(\mathrm{~s}, 3 \mathrm{H}), 3.81(\mathrm{~s}$, $3 \mathrm{H}), 4.92(\mathrm{~d}, J=8.99 \mathrm{~Hz}, 1 \mathrm{H}), 5.76(\mathrm{~d}, J=8.99 \mathrm{~Hz}, 1 \mathrm{H}), 6.89(\mathrm{~d}$, $J=8.80 \mathrm{~Hz}, 2 \mathrm{H}), 7.23(\mathrm{~d}, J=8.80 \mathrm{~Hz}, 2 \mathrm{H})$. Found: C, 57.88; H, $6.04 ; \mathrm{N}, 3.86 \%$. Calcd for $\mathrm{C}_{17} \mathrm{H}_{21} \mathrm{NO}_{7}: \mathrm{C}, 58.11 ; \mathrm{H}, 6.02 ; \mathrm{N}$, $3.99 \%$. When the $\alpha$-proton of the ester group was irradiated, $12.4 \%$ NOE was observed for the $\beta$-proton.

A General Procedure for the Preparation of N,N-Diprotected Glycine Methyl Esters (3a-3f). A solution of methyl Bocglycinate $(482 \mathrm{mg}, 2.55 \mathrm{mmol}), \mathrm{Boc}_{2} \mathrm{O}(1.113 \mathrm{~g}, 5.10 \mathrm{mmol})$, and DMAP $(93 \mathrm{mg}, 0.761 \mathrm{mmol})$ in dry acetonitrile $\left(\mathrm{CH}_{3} \mathrm{CN}\right)(5 \mathrm{~mL})$ was stirred at $55^{\circ} \mathrm{C}$ for $22 \mathrm{~h}$. The solvent was then removed in vacuo to give a residue, which was partitioned between EtOAc and a saturated solution of $\mathrm{NH}_{4} \mathrm{Cl}$. The aqueous solution was extracted with EtOAc. The combined extracts were washed with brine and dried over $\mathrm{MgSO}_{4}$, and concentrated under reduced pressure. The residue was subjected to silica gel column chromatography (eluent; hexane $/$ EtOAc $=10 / 1, \mathrm{v} / \mathrm{v}$ ) to afford methyl $N, N$-diBoc-glycinate (3a) in quantitative yield (734 mg). Mp 59.0-59.5 ${ }^{\circ} \mathrm{C}$ (hexane); IR (KBr): 3001, 2978, 1756, 1731, 1694, 1435, 1412, 1383, 1368, $1335,1231,1146,1127,991,962,897,862,848,788,768 \mathrm{~cm}^{-1}$; 
${ }^{1} \mathrm{HNMR}\left(400 \mathrm{MHz}, \mathrm{CDCl}_{3}\right): \delta 1.50(\mathrm{~s}, 18 \mathrm{H}), 3.75(\mathrm{~s}, 3 \mathrm{H}), 4.34(\mathrm{~s}$, $2 \mathrm{H}$ ). Found: $\mathrm{C}, 53.70 ; \mathrm{H}, 7.95 ; \mathrm{N}, 4.74 \%$. Calcd for $\mathrm{C}_{13} \mathrm{H}_{23} \mathrm{NO}_{6}$ : $\mathrm{C}$, 53.97; H, 8.01; N, 4.84\%.

The physical and spectral data of compounds $\mathbf{3 b} \mathbf{- 3 f}$ are shown in the following.

Methyl $\boldsymbol{N}$-Boc- $\boldsymbol{N}$-Z-glycinate (3b): $\quad$ Oil; IR (neat): 2979 , $1801,1761,1699,1456,1439,1415,1370,1343,1258,1217$, $1182,1153,1107,1004,951,916,855,780,752,698 \mathrm{~cm}^{-1}$; ${ }^{1} \mathrm{HNMR}\left(300 \mathrm{MHz}, \mathrm{CDCl}_{3}\right): \delta 1.46(\mathrm{~s}, 9 \mathrm{H}), 3.71(\mathrm{~s}, 3 \mathrm{H}), 4.41(\mathrm{~s}$, $2 \mathrm{H}), 5.24(\mathrm{~s}, 2 \mathrm{H}), 7.30-7.40(\mathrm{~m}, 5 \mathrm{H})$. HRMS $\left(\mathrm{FAB}^{+}\right)\left(\mathrm{M}^{+}+1\right)$ : Found: $m / z$ 324.1451. Calcd for $\mathrm{C}_{16} \mathrm{H}_{22} \mathrm{NO}_{6}$ : 324.1447 .

Methyl $\quad \mathrm{N}$-Boc- $\mathrm{N}$-2,2,2-trichloroethoxycarbonylglycinate (3c): $\quad$ Mp 51.0-51.5 ${ }^{\circ} \mathrm{C}$ (heptane); IR (KBr): 2982, 2957, 1812, $1756,1708,1439,1416,1371,1346,1255,1221,1156,1062$, 1019, 956, 855, 816, 778, $720 \mathrm{~cm}^{-1}$; ${ }^{1} \mathrm{HNMR}\left(400 \mathrm{MHz}, \mathrm{CDCl}_{3}\right)$ : $\delta 1.54(\mathrm{~s}, 9 \mathrm{H}), 3.76(\mathrm{~s}, 3 \mathrm{H}), 4.47(\mathrm{~s}, 2 \mathrm{H}), 4.82(\mathrm{~s}, 2 \mathrm{H})$. Found: $\mathrm{C}$, $35.99 ; \mathrm{H}, 4.44 ; \mathrm{N}, 3.82 \%$. Calcd for $\mathrm{C}_{11} \mathrm{H}_{16} \mathrm{NO}_{6} \mathrm{Cl}_{3}$ : C, 36.24; $\mathrm{H}$, $4.42 ; \mathrm{N}, 3.84 \%$.

Methyl $\mathbf{N}$-Boc- $\boldsymbol{N}$-methoxycarbonylglycinate (3d): Oil; IR (neat): 2980, 2958, 1804, 1764, 1703, 1444, 1410, 1369, 1347, 1224, 1155, 1112, 1010, 948, 855, $783 \mathrm{~cm}^{-1}$; ${ }^{1} \mathrm{H}$ NMR $(300 \mathrm{MHz}$, $\left.\mathrm{CDCl}_{3}\right): \delta 1.50(\mathrm{~s}, 9 \mathrm{H}), 3.76(\mathrm{~s}, 3 \mathrm{H}), 3.84(\mathrm{~s}, 3 \mathrm{H}), 4.40(\mathrm{~s}$, $2 \mathrm{H})$. HRMS $\left(\mathrm{FAB}^{+}\right)\left(\mathrm{M}^{+}+1\right)$ Found: $m / z$ 248.1134. Calcd for $\mathrm{C}_{10} \mathrm{H}_{18} \mathrm{NO}_{6}: 248.1134$.

Methyl $\boldsymbol{N}$-Boc- $\boldsymbol{N}$-ethoxycarbonylglycinate (3e): Oil; IR (neat): 2981, 1803, 1736, 1724, 1700, 1478, 1439, 1418, 1371, 1344, 1223, 1156, 1108, 1025, 959, 926, 893, 856, $782 \mathrm{~cm}^{-1}$; ${ }^{1} \mathrm{HNMR}\left(300 \mathrm{MHz}, \mathrm{CDCl}_{3}\right): \delta 1.32(\mathrm{t}, J=7.15 \mathrm{~Hz}, 3 \mathrm{H}), 1.50(\mathrm{~s}$, $9 \mathrm{H}), 3.76(\mathrm{~s}, 3 \mathrm{H}), 4.28(\mathrm{q}, J=7.15 \mathrm{~Hz}, 2 \mathrm{H}), 4.40$ (s, 2H). HRMS $\left(\mathrm{FAB}^{+}\right)\left(\mathrm{M}^{+}+1\right)$ : Found: $m / z$ 262.1296. Calcd for $\mathrm{C}_{11} \mathrm{H}_{20} \mathrm{NO}_{6}$ : 262.1291.

Methyl $N$-Boc- $N$-phenoxycarbonylglycinate (3f): Mp $56.5-$ $57.5^{\circ} \mathrm{C}$ (hexane); IR (KBr): 2980, 1810, 1753, 1712, 1594, 1494, 1413, 1369, 1347, 1201, 1148, 1099, 1075, 1001, 959, 855, 777, $754 \mathrm{~cm}^{-1} ;{ }^{1} \mathrm{HNMR}\left(400 \mathrm{MHz}, \mathrm{CDCl}_{3}\right): \delta 1.54(\mathrm{~s}, 9 \mathrm{H}), 3.79(\mathrm{~s}$, $3 \mathrm{H}), 4.52$ (s, 2H), 7.14-7.40 (m, 5H). Found: C, 57.94; H, 6.19; N, 4.54\%. Calcd for $\mathrm{C}_{15} \mathrm{H}_{19} \mathrm{NO}_{6}$ : C, 58.24; $\mathrm{H}, 6.19 ; \mathrm{N}, 4.53 \%$.

4-( $N$-Boc- $N$-Z-amino)butanal (4h). $\quad 4$ - $(N$-Boc- $N$-Z-amino)butanal (4h) in Table 3 was prepared starting from 4-amino-1butanol.

To a solution of oxalyl chloride $(0.96 \mathrm{~mL}, 11 \mathrm{mmol})$ in dry $\mathrm{CH}_{2} \mathrm{Cl}_{2}(25 \mathrm{~mL})$ added DMSO $(2.13 \mathrm{~mL}, 30 \mathrm{mmol})^{21}$ at $-78^{\circ} \mathrm{C}$ under $\mathrm{N}_{2}$. After $15 \mathrm{~min}$, a solution of $17(3.24 \mathrm{~g}, 10 \mathrm{mmol})$ in $\mathrm{CH}_{2} \mathrm{Cl}_{2}(10 \mathrm{~mL})$ was added and the solution was stirred for $15 \mathrm{~min}$ at that temperature. After addition of TEA $(10.5 \mathrm{~mL}, 75 \mathrm{mmol})$ the mixture was gradually warmed to room temperature and then stirred for $1 \mathrm{~h}$. The solvent was removed in vacuo to give a residue, which was partitioned between EtOAc, $\mathrm{Et}_{2} \mathrm{O}$, and water. The organic layer was dried over $\mathrm{MgSO}_{4}$ and evaporated in vacuo to afford a residual oil which was subjected to silica gel column chromatography (eluent, hexane/EtOAc $=2 / 1, \mathrm{v} / \mathrm{v}$ ). Compound 4h was obtained in $90 \%$ yield $(2.90 \mathrm{~g})$. Oil; IR (neat): 2979,2936 , 2823, 2723, 1790, 1724, 1498, 1455, 1369, 1342, 1294, 1264, $1202,1154,1119,1065,1031,1003,854,779,752,669 \mathrm{~cm}^{-1}$; ${ }^{1} \mathrm{HNMR}\left(300 \mathrm{MHz}, \mathrm{CDCl}_{3}\right): \delta 1.47(\mathrm{~s}, 9 \mathrm{H}), 1.86-1.95(\mathrm{~m}, 2 \mathrm{H})$, $2.43(\mathrm{t}, J=7.33 \mathrm{~Hz}, 2 \mathrm{H}), 3.69(\mathrm{t}, J=7.15 \mathrm{~Hz}, 2 \mathrm{H}), 5.22(\mathrm{~s}, 2 \mathrm{H})$, 7.33-7.38 (m, 5H), $9.71(\mathrm{~s}, 1 \mathrm{H})$. HRMS $\left(\mathrm{FAB}^{+}\right)\left(\mathrm{M}^{+}+1\right)$ : Found: $m / z$ 322.1656. Calcd for $\mathrm{C}_{17} \mathrm{H}_{24} \mathrm{NO}_{5}$ : 322.1654.

Methyl 2-(Boc-amino)-3-(t-butoxycarbonyloxy)-3-phenylpropanoate (6). To a solution of 3a $(277 \mathrm{mg}, 0.79 \mathrm{mmol})$ in dry THF $(4 \mathrm{~mL})$ was added 1.1 molar equivalents of LDA at $-78{ }^{\circ} \mathrm{C}$ under $\mathrm{N}_{2}$. After the solution was stirred at that temperature for $1 \mathrm{~h}$, a solution of benzaldehyde (4i) $(92 \mathrm{mg}, 0.87 \mathrm{mmol})$ in THF $(4 \mathrm{~mL})$ was added dropwise at $-78^{\circ} \mathrm{C}$ and the solution was kept at $-78^{\circ} \mathrm{C}$ for $1 \mathrm{~h}$. After quenching the reaction at that temperature with $0.5 \mathrm{M} \mathrm{HCl}$ in $\mathrm{MeOH}$, the solvent was removed in vacuo to give a residue, which was partitioned between EtOAc and water. The aqueous layer was extracted with EtOAc. The combined extracts were washed with brine dried over $\mathrm{MgSO}_{4}$, and evaporated in vacuo. The residue was subjected to preparative TLC $\left(\mathrm{SiO}_{2}\right.$, hexane/EtOAc $\left.=7 / 1, \mathrm{v} / \mathrm{v}\right)$ to afford methyl 2-(Bocamino)-3-( $t$-butoxycarbonyloxy)-3-phenylpropanoate (6) in $85 \%$ yield $(265 \mathrm{mg})$. Mp 84.0-85.0 ${ }^{\circ} \mathrm{C}$ (EtOAc-hexane); IR (KBr): 3404, 2979, 1750, 1702, 1519, 1369, 1287, 1252, 1160, 1090, 1029, 968, 872, 760, $709 \mathrm{~cm}^{-1} ;{ }^{1} \mathrm{H}$ NMR $\left(300 \mathrm{MHz}, \mathrm{CDCl}_{3}\right): \delta$ $1.41(\mathrm{~s}, 9 \mathrm{H}), 1.47(\mathrm{~s}, 9 \mathrm{H}), 3.70(\mathrm{~s}, 3 \mathrm{H}), 4.95-5.05(\mathrm{~m}, 2 \mathrm{H}), 5.93(\mathrm{~d}$, $J=4.03 \mathrm{~Hz}, 1 \mathrm{H}), 7.29-7.39$ (m, 5H). Found: C, 60.58; H, 7.51; N, $3.45 \%$. Calcd for $\mathrm{C}_{20} \mathrm{H}_{29} \mathrm{NO}_{7}: \mathrm{C}, 60.75 ; \mathrm{H}, 7.39 ; \mathrm{N}, 3.54 \%$.

(3S,5S)-3-( $\mathrm{N}$-Z-amino)-5-( $t$-butyldiphenylsiloxy)methyl- $\gamma$-lactone (7). To compound $12(180 \mathrm{mg}, 0.38 \mathrm{mmol})$ in $\mathrm{CH}_{2} \mathrm{Cl}_{2}$ $(0.5 \mathrm{~mL})$ was added TFA $(0.5 \mathrm{~mL})$ at $0{ }^{\circ} \mathrm{C}$ under $\mathrm{N}_{2}$. The solution was stirred for $1 \mathrm{~h}$ at room temperature, and the solvent was removed in vacuo to afford a residue, which was dissolved in THF $(3 \mathrm{~mL})$. To the solution was added a solution of TEA (116 mg, $1.15 \mathrm{mmol})$ in THF $(0.5 \mathrm{~mL})$ followed by the addition of $N$-benzyloxycarbonyloxysuccinimide (Z-ONSu) (144 mg, $0.58 \mathrm{mmol}$ ). The solution was allowed to stir for $2 \mathrm{~h}$ at room temperature. After evaporation of the solvent the residue was partitioned between EtOAc and water. The EtOAc was washed with brine, dried over $\mathrm{MgSO}_{4}$, and evaporated in vacuo to give a residue, which was subjected to preparative TLC $\left(\mathrm{SiO}_{2}\right.$, hexane/ EtOAc $=3 / 1, \mathrm{v} / \mathrm{v})$. The desired product 7 was obtained in $80 \%$ yield $(155 \mathrm{mg})$. Oil; $[\alpha]_{\mathrm{D}}^{25}+3.96^{\circ}\left(c 0.88\right.$, EtOH) $\left[\mathrm{Lit},[\alpha]_{\mathrm{D}}^{25}+3.9^{\circ}\right.$ (c 2.1, EtOH)]; ${ }^{16}$ IR (neat): 3331, 3070, 2931, 2858, 1786, 1713, $1588,1525,1471,1455,1428,1362,1328,1259,1198,1113$, 1058, 1027, 997, 935, 907, 823, 777, 742, $703 \mathrm{~cm}^{-1} ;{ }^{1} \mathrm{HNMR}$ $\left(400 \mathrm{MHz}, \mathrm{CDCl}_{3}\right): \delta 1.05(\mathrm{~s}, 9 \mathrm{H}), 2.06-2.18(\mathrm{~m}, 1 \mathrm{H}), 2.66-2.74$ (m, 1H), 3.74 (dd, $J=4.04,11.55 \mathrm{~Hz}, 1 \mathrm{H}), 3.90$ (dd, $J=2.38$, $11.55 \mathrm{~Hz}, 1 \mathrm{H}), 4.53-4.59$ (br, 2H), 5.13 (s, 2H), 5.30 (br, 1H), 7.30$7.46(\mathrm{~m}, 11 \mathrm{H}), 7.63-7.67(\mathrm{~m}, 4 \mathrm{H})$. HRMS $\left(\mathrm{FAB}^{+}\right)\left(\mathrm{M}^{+}+1\right)$ : Found: $m / z$ 504.2197. Calcd for $\mathrm{C}_{29} \mathrm{H}_{34} \mathrm{NO}_{5} \mathrm{Si}: 504.2206$.

3-Boc-4-methoxycarbonyl-5-(2,2-dimethyl-[1,3]dioxolane-4yl)-cis-4,5-oxazolidin-2-one (8a and $\left.8 \mathrm{a}^{\prime}\right)$. To a solution of $\mathbf{3 f}$ (309 $\mathrm{mg}, 1.0 \mathrm{mmol})$ in dry THF $(6 \mathrm{~mL})$ was added 1.1 molar equivalents of LDA $(1.1 \mathrm{mmol})$ at $-78^{\circ} \mathrm{C}$ and the solution was stirred for $30 \mathrm{~min}$ at that temperature. A solution of $(R)-O-2,3-$ isopropylideneglycelaldehyde $(195 \mathrm{mg}, 1.5 \mathrm{mmol})$ in dry THF $(5 \mathrm{~mL})$ was added dropwise at $-78^{\circ} \mathrm{C}$ followed by the addition of $\mathrm{Ti}\left(\mathrm{O}^{i} \mathrm{Pr}\right)_{4}(427 \mathrm{mg}, 1.5 \mathrm{mmol})$. The resulting solution was allowed to stir for $2 \mathrm{~h}$ at $-78^{\circ} \mathrm{C}$ and quenched with $1 \mathrm{M} \mathrm{AcOH}$ in THF at that temperature. After evaporation of the solvent, the residue was partitioned between EtOAc and water. The EtOAc solution was washed with brine, dried over $\mathrm{MgSO}_{4}$, and concentrated under reduced pressure to afford a residue. The crude product was separated by preparative TLC $\left(\mathrm{SiO}_{2}\right.$, hexane/EtOAc $\left.=3 / 1, \mathrm{v} / \mathrm{v}\right)$ to afford the oxazolidinone derivatives 8a (oily form, $138 \mathrm{mg}$ ) in $40 \%$ and $\mathbf{8 a ^ { \prime }}$ (crystalline form, $<86 \mathrm{mg}$ ) in $<25 \%$ yields, respectively. 8a: oil; IR (neat): 2985, 2937, 1828, 1758, 1730, 1479, 1457, 1438, 1372, 1333, 1255, 1215, 1157, 1068, 846, $771 \mathrm{~cm}^{-1}$; ${ }^{1} \mathrm{HNMR}\left(300 \mathrm{MHz}, \mathrm{CDCl}_{3}\right): \delta 1.31(\mathrm{~s}, 3 \mathrm{H}), 1.42(\mathrm{~s}, 3 \mathrm{H}), 1.50(\mathrm{~s}$, $9 \mathrm{H}), 3.81(\mathrm{~s}, 3 \mathrm{H}), 3.99(\mathrm{dd}, J=3.67,8.62 \mathrm{~Hz}, 1 \mathrm{H}), 4.08-4.16(\mathrm{~m}$, $2 \mathrm{H}), 4.50(\mathrm{~m}, 1 \mathrm{H}), 4.84(\mathrm{~d}, J=8.62 \mathrm{~Hz}, 1 \mathrm{H})$. HRMS $\left(\mathrm{FAB}^{+}\right)$ $\left(\mathrm{M}^{+}+1\right)$ : Found: $m / z$ 346.1505. Calcd for $\mathrm{C}_{15} \mathrm{H}_{24} \mathrm{NO}_{8}: 346.1502$. When the proton at $\mathrm{C}-4$ position was irradiated, $15.2 \%$ and $1.3 \%$ 
NOE were observed for the proton at $\mathrm{C}-5$ and the methyl protons of the ester group, respectively. 8a': mp 189.0-191.0 ${ }^{\circ} \mathrm{C}$ (EtOAchexane). IR (KBr): 2992, 2939, 2899, 1807, 1759, 1723, 1458, 1369, 1337, 1218, 1158, 1103, 1070, 1033, 992, 973, 916, 862, $839,785,766 \mathrm{~cm}^{-1}$; ${ }^{1} \mathrm{H}$ NMR $\left(400 \mathrm{MHz}, \mathrm{CDCl}_{3}\right): \delta 1.34(\mathrm{~s}, 3 \mathrm{H})$, $1.41(\mathrm{~s}, 3 \mathrm{H}), 1.51(\mathrm{~s}, 9 \mathrm{H}), 3.79(\mathrm{~s}, 3 \mathrm{H}), 3.96(\mathrm{dd}, J=6.24,8.62 \mathrm{~Hz}$, $1 \mathrm{H}), 4.12(\mathrm{dd}, J=6.79,8.62 \mathrm{~Hz}, 1 \mathrm{H}), 4.29$ (ddd, $J=3.85,6.24$, $6.79 \mathrm{~Hz}, 1 \mathrm{H}), 4.58(\mathrm{dd}, J=3.85,8.80 \mathrm{~Hz}, 1 \mathrm{H}), 4.74(\mathrm{~d}, J=$ $8.80 \mathrm{~Hz}, 1 \mathrm{H})$. Found: C, 51.89; H, 6.77; N, 4.09\%. Calcd for $\mathrm{C}_{15} \mathrm{H}_{23} \mathrm{NO}_{8}$ : $\mathrm{C}, 52.17 ; \mathrm{H}, 6.71 ; \mathrm{N}, 4.06 \%$. When the proton at $\mathrm{C}-4$ was irradiated, $11.4 \%$ and $1.0 \%$ NOE were observed for the proton at $\mathrm{C}-5$ and methyl protons of the ester group, respectively.

Methyl (4S)-2-( $N$-Boc-amino)-4,5-isopropylidenedioxy-2pentenoate (Mixture of $\boldsymbol{E}$ - and $\boldsymbol{Z}$-9). To a solution of $8 \mathrm{a}$ $(65 \mathrm{mg}, 0.188 \mathrm{mmol})$ in dry THF $(4 \mathrm{~mL})$ was added 3.0 molar equivalents of LiHMDS $(0.564 \mathrm{mmol})$ at $-10^{\circ} \mathrm{C}$ under $\mathrm{N}_{2}$ and the solution was stirred for $5 \mathrm{~min}$ at that temperature. The reaction was quenched with $1 \mathrm{M} \mathrm{AcOH}$ in THF and the solvent was removed in vacuo to give a residue, which was partitioned between EtOAc and water. The EtOAc solution was washed with brine, dried over $\mathrm{MgSO}_{4}$, and evaporated in vacuo to afford a residue, which was subjected to preparative TLC $\left(\mathrm{SiO}_{2}\right.$, hexane/EtOAc $\left.=3 / 1, \mathrm{v} / \mathrm{v}\right)$. The desired product 9 was obtained as a mixture of $\mathrm{E}$ and $\mathrm{Z}$ isomers in a ratio of 78:22 in 70\% yield (40 mg). IR (neat): 3417 , 3340, 2983, 2935, 2875, 1725, 1653, 1512, 1456, 1439, 1369, 1345, 1246, 1158, 1058, 1021, 845, $761 \mathrm{~cm}^{-1}$; ${ }^{1} \mathrm{H}$ NMR $(300 \mathrm{MHz}$, $\left.\mathrm{CDCl}_{3}\right)$ : E isomer: $\delta 1.39(\mathrm{~s}, 3 \mathrm{H}), 1.45(\mathrm{~s}, 3 \mathrm{H}), 1.47(\mathrm{~s}, 9 \mathrm{H}), 3.65$ $(\mathrm{dd}, J=7.52,7.89 \mathrm{~Hz}, 1 \mathrm{H}), 3.85(\mathrm{~s}, 3 \mathrm{H}), 4.25(\mathrm{dd}, J=6.60$, $8.05 \mathrm{~Hz}, 1 \mathrm{H}), 5.34$ (dd, $J=6.60,13.94 \mathrm{~Hz}, 1 \mathrm{H}), 6.70$ (br s, $1 \mathrm{H})$, $6.88(\mathrm{~d}, J=7.34 \mathrm{~Hz}, 1 \mathrm{H}) . \mathrm{Z}$ isomer: $\delta 1.39(\mathrm{~s}, 3 \mathrm{H}), 1.45(\mathrm{~s}, 3 \mathrm{H})$, $1.46(\mathrm{~s}, 9 \mathrm{H}), 3.80(\mathrm{~s}, 3 \mathrm{H}), 3.81-3.86(\mathrm{~m}, 1 \mathrm{H}), 4.34(\mathrm{dd}, J=6.60$, $8.62 \mathrm{~Hz}, 1 \mathrm{H}), 4.85(\mathrm{dd}, J=6.60,14.67 \mathrm{~Hz}, 1 \mathrm{H}), 6.40(\mathrm{~d}, J=$ $8.25 \mathrm{~Hz}, 1 \mathrm{H}), 6.44$ (br s, 1H). HRMS $\left(\mathrm{FAB}^{+}\right)\left(\mathrm{M}^{+}+1\right)$ Found: 302.1599. Calcd for $\mathrm{C}_{14} \mathrm{H}_{24} \mathrm{NO}_{6}: 302.1604$.

(4S)-2-( $N$-Boc-amino)-5-hydroxymethylbutenolide (10). A solution of compound $9(\mathrm{E} / \mathrm{Z}=78 / 22,160 \mathrm{mg}, 0.53 \mathrm{mmol})$ and $1 \mathrm{M} \mathrm{HCl}$ in $\mathrm{MeOH}(2.0 \mathrm{~mL})$ was stirred for $1 \mathrm{~d}$ under air. After neutralization with $\mathrm{NaHCO}_{3}$ solution at $0{ }^{\circ} \mathrm{C}$, the solvent was removed in vacuo to give a residue, which was partitioned between EtOAc and water. The organic solution was washed with brine, dried over $\mathrm{MgSO}_{4}$, and concentrated under reduced pressure. The residue was purified with preparative $\mathrm{TLC}\left(\mathrm{SiO}_{2}\right.$, hexane/ EtOAc $=1 / 1, \mathrm{v} / \mathrm{v})$ to afford the product $\mathbf{1 0}$ in $61 \%$ yield (74 mg). Mp 123.0-124.0 ${ }^{\circ} \mathrm{C}$ (EtOAc-hexane); $[\alpha]_{\mathrm{D}}^{25}+18.14^{\circ}(c$ $\left.0.19, \mathrm{CHCl}_{3}\right)$; IR (KBr): $3425,2979,1767,1662,1538,1456$, 1370, 1344, 1315, 1241, 1160, 1075, 1045, 1016, 934, 888, 849, $770 \mathrm{~cm}^{-1} ;{ }^{1} \mathrm{H} \mathrm{NMR}\left(300 \mathrm{MHz}, \mathrm{CDCl}_{3}\right): \delta 1.50(\mathrm{~s}, 9 \mathrm{H}), 2.30-2.44$ (br, $1 \mathrm{H}), 3.71(\mathrm{dd}, J=5.69,12.29 \mathrm{~Hz}, 1 \mathrm{H}), 3.97(\mathrm{dd}, J=3.30$, $12.29 \mathrm{~Hz}, 1 \mathrm{H}), 5.14(\mathrm{~m}, 1 \mathrm{H}), 6.82-6.98(\mathrm{br}, 1 \mathrm{H}), 7.04-7.08(\mathrm{br}$, $1 \mathrm{H})$. Found: $\mathrm{C}, 52.12 ; \mathrm{H}, 6.63 ; \mathrm{N}, 6.05 \%$. Calcd for $\mathrm{C}_{10} \mathrm{H}_{15} \mathrm{NO}_{5}$ : C, $52.40 ; \mathrm{H}, 6.60 ; \mathrm{N}, 6.11 \%$.

(4S)-2-( $N$-Boc-amino)-5-(t-butyldiphenylsiloxy)methylbutenolide (11). A solution of $\mathbf{1 0}(85 \mathrm{mg}, 0.37 \mathrm{mmol})$, TBDPS-Cl (123 mg, $0.45 \mathrm{mmol}$ ), and imidazole (76 mg, $1.12 \mathrm{mmol})$ in DMF $(3 \mathrm{~mL})$ was allowed to stir for $4 \mathrm{~h}$ at room temperature. The reaction solution was diluted with water and extracted with ether. The ethereal solution was washed with brine, dried over $\mathrm{MgSO}_{4}$, and evaporated in vacuo to give a residue which was subjected to preparative TLC $\left(\mathrm{SiO}_{2}\right.$, hexane/EtOAc $\left.=3 / 1, \mathrm{v} / \mathrm{v}\right)$. The desired product 11 was obtained as oil in $80 \%$ yield $(132 \mathrm{mg}) .[\alpha]_{\mathrm{D}}^{25}$ $+25.84^{\circ}$ (c 1.18, EtOH); IR (neat): 3421, 3317, 3134, 3071, 3049, 2959, 2931, 2893, 2858, 2741, 2344, 1961, 1768, 1734, 1667,
$1589,1519,1472,1428,1392,1369,1344,1316,1237,1158$, $1115,1085,1045,1010,937,904,857,823,772,742,703 \mathrm{~cm}^{-1}$; ${ }^{1} \mathrm{HNMR}\left(300 \mathrm{MHz}, \mathrm{CDCl}_{3}\right): \delta 1.03(\mathrm{~s}, 9 \mathrm{H}), 1.50(\mathrm{~s}, 9 \mathrm{H}), 3.76(\mathrm{dd}$, $J=4.95,11.00 \mathrm{~Hz}, 1 \mathrm{H}), 3.89(\mathrm{dd}, J=4.22,11.00 \mathrm{~Hz}, 1 \mathrm{H}), 5.05-$ $5.09(\mathrm{~m}, 1 \mathrm{H}), 6.77(\mathrm{br}, 1 \mathrm{H}), 6.97(\mathrm{br}, 1 \mathrm{H}), 7.36-7.42(\mathrm{~m}, 5 \mathrm{H})$, 7.62-7.66 (m, 5H). HRMS $\left(\mathrm{FAB}^{+}\right)\left(\mathrm{M}^{+}+1\right)$ : Found: $m / z$ 468.2208. Calcd for $\mathrm{C}_{26} \mathrm{H}_{34} \mathrm{NO}_{5} \mathrm{Si}$ : 468.2206.

(3S,5S)-3-( $N$-Boc-amino)-5-( $t$-butyldiphenylsiloxy)methyl- $\gamma$ lactone (12). Compound 11 (94 mg, $0.21 \mathrm{mmol}$ ) was hydrogenated over $20 \%$ palladium hydroxide on carbon $(12 \mathrm{mg})$ in $\mathrm{EtOH}(3 \mathrm{~mL})$ at room temperature under hydrogen atmosphere for $10 \mathrm{~h}$. Then, the catalyst was filtered through a pad of celite, and the filtrate was removed in vacuo. The residual oil was subjected to preparative TLC $\left(\mathrm{SiO}_{2}\right.$, hexane/EtOAc $\left.=3 / 1, \mathrm{v} / \mathrm{v}\right)$ to afford compound 12 in $89 \%$ yield $(88 \mathrm{mg})$. Oil; $[\alpha]_{\mathrm{D}}^{25}+3.56^{\circ}$ (c 1.25, EtOH); IR (neat): 3351, 3071, 3050, 2964, 2931, 2894, 2859, 1962, 1894, 1784, 1713, 1589, 1510, 1473, 1456, 1428, 1391, 1367, 1328, 1291, 1254, 1164, 1114, 1051, 1030, 978, 936, 911, 871, 823, 742, $704 \mathrm{~cm}^{-1} ;{ }^{1} \mathrm{HNMR}\left(400 \mathrm{MHz}, \mathrm{CDCl}_{3}\right): \delta 1.06(\mathrm{~s}$, $9 \mathrm{H}), 1.46(\mathrm{~s}, 9 \mathrm{H}), 2.03-2.11(\mathrm{~m}, 1 \mathrm{H}), 2.66-2.81(\mathrm{br}, 1 \mathrm{H}), 3.74$ (dd, $J=3.90,11.50 \mathrm{~Hz}, 1 \mathrm{H}), 3.89(\mathrm{dd}, J=2.93,11.50 \mathrm{~Hz}, 1 \mathrm{H}), 4.44$ $5.16(\mathrm{br}, 1 \mathrm{H}), 4.48-4.55(\mathrm{~m}, 2 \mathrm{H}), 7.36-7.47(\mathrm{~m}, 6 \mathrm{H}), 7.64-7.68$ $(\mathrm{m}, 4 \mathrm{H})$. HRMS $\left(\mathrm{FAB}^{+}\right)\left(\mathrm{M}^{+}+1\right)$ : Found: $m / z$ 470.2360. Calcd for $\mathrm{C}_{26} \mathrm{H}_{36} \mathrm{NO}_{5} \mathrm{Si}: 470.2363$.

A General Procedure for Coupling Reaction of Boc-Amino Acid with cis-4,5-Oxazolidinone Derivatives (13a-13k'). A solution of 3-Boc-4-(methoxycarbonyl)-5-phenyl-cis-4,5-oxazolidin-2-one (2i) $(321 \mathrm{mg}, 1.0 \mathrm{mmol})$ in $99 \%$ formic acid $(20 \mathrm{~mL})$ was allowed to stand for $40 \mathrm{~min}$. Evaporation of the solvent afforded the $\mathrm{N}$-deprotected oxazolidinone derivative in quantitative yield (mp $147-148{ }^{\circ} \mathrm{C}$ from EtOAc-hexane). To the resulting oxazolidinone $(221 \mathrm{mg}, 1.0 \mathrm{mmol})$ in dry THF $(5 \mathrm{~mL})$ was added dropwise 1.1 molar equivalents of LDA at $-78^{\circ} \mathrm{C}$ under $\mathrm{N}_{2}$. After $5 \mathrm{~min}$ a solution of Boc-Ala-ONSu (429 mg, $1.1 \mathrm{mmol})$ in THF $\left(5 \mathrm{~mL}\right.$ ) was added at $-78^{\circ} \mathrm{C}$ followed by the addition of a solution of DMAP $(12 \mathrm{mg}, 0.1 \mathrm{mmol})$ in dry THF $(2 \mathrm{~mL})$. The solution was then warmed to $-10^{\circ} \mathrm{C}$ and kept at that temperature for $3 \mathrm{~h}$. After quenching the reaction with $1 \mathrm{M} \mathrm{AcOH}$ in $\mathrm{THF}$ at $-78^{\circ} \mathrm{C}$, the solvent was removed in vacuo to give a residue, which was partitioned between EtOAc and water. The aqueous layer was extracted with EtOAc. The combined EtOAc extracts were washed with a solution of $10 \% \mathrm{NaHCO}_{3}$ and brine, dried over $\mathrm{MgSO}_{4}$, and concentrated under reduced pressure. The residue was subjected to preparative TLC $\left(\mathrm{SiO}_{2}\right.$, hexane/EtOAc $\left.=2 / 1, \mathrm{v} / \mathrm{v}\right)$ to give $\mathbf{1 3 i}$ as major product and $\mathbf{1 3 i} \mathbf{i}^{\prime}$ as minor one in $51 \%(199 \mathrm{mg})$ and $45 \%$ $(177 \mathrm{mg})$ yields, respectively. 13i: $\mathrm{mp} 160.0-161.0^{\circ} \mathrm{C}$ (EtOAchexane); $[\alpha]_{\mathrm{D}}^{25}-54.03^{\circ}$ (c 0.74, EtOH); IR (KBr): 3423, 2985, 2953, 1806, 1748, 1722, 1702, 1503, 1368, 1340, 1243, 1216, $1196,1143,1020,757,701 \mathrm{~cm}^{-1} ;{ }^{1} \mathrm{HNMR}\left(400 \mathrm{MHz}, \mathrm{CDCl}_{3}\right): \delta$ $1.45(\mathrm{~s}, 9 \mathrm{H}), 1.48(\mathrm{~d}, J=7.07 \mathrm{~Hz}, 3 \mathrm{H}), 3.20(\mathrm{~s}, 3 \mathrm{H}), 5.02(\mathrm{~d}$, $J=8.05 \mathrm{~Hz}, 1 \mathrm{H}), 5.14(\mathrm{~d}, J=9.03 \mathrm{~Hz}, 1 \mathrm{H}), 5.40(\mathrm{dq}, J=7.07$, $8.05 \mathrm{~Hz}, 1 \mathrm{H}), 5.82(\mathrm{~d}, J=9.03 \mathrm{~Hz}, 1 \mathrm{H}), 7.28-7.44(\mathrm{~m}, 2 \mathrm{H})$, 7.36-7.44 (m, 3H). Found: C, 58.08; H, 6.14; N, 7.10\%. Calcd for $\mathrm{C}_{19} \mathrm{H}_{24} \mathrm{~N}_{2} \mathrm{O}_{7}$ : C, 58.15; H, 6.17; N, 7.14\%. 13i': mp 145.0$146.0^{\circ} \mathrm{C}$ (EtOAc-hexane); $[\alpha]_{\mathrm{D}}^{25}-38.59^{\circ}$ (c $\left.0.68, \mathrm{EtOH}\right) ; \mathrm{IR}$ (KBr): 3374, 2981, 2934, 1784, 1747, 1725, 1692, 1627, 1521, $1359,1249,1215,1200,1166,1140,1012,760,701 \mathrm{~cm}^{-1}$; ${ }^{1} \mathrm{HNMR}\left(300 \mathrm{MHz}, \mathrm{CDCl}_{3}\right): \delta 1.42-1.50(\mathrm{br}, 3 \mathrm{H}), 1.44(\mathrm{~s}, 9 \mathrm{H})$, $3.24(\mathrm{~s}, 3 \mathrm{H}), 5.03(\mathrm{~d}, J=8.78 \mathrm{~Hz}, 1 \mathrm{H}), 5.22-5.34(\mathrm{br}, 1 \mathrm{H}), 5.39$ $5.58(\mathrm{br}, 1 \mathrm{H}), 5.81(\mathrm{~d}, J=8.78 \mathrm{~Hz}, 1 \mathrm{H}), 7.28-7.35(\mathrm{~m}, 2 \mathrm{H}), 7.36-$ 7.43 (m, 3H). Found: C, 58.23; H, 6.31; N, 7.05\%. Calcd for $\mathrm{C}_{19} \mathrm{H}_{24} \mathrm{~N}_{2} \mathrm{O}_{7}$ : C, 58.15; H, 6.17; N, 7.14\%. 
The physical and spectral data of compounds $\mathbf{1 3} \mathbf{a}-13 \mathbf{h}^{\prime}$ and $\mathbf{1 3} \mathbf{j}-\mathbf{1 3} \mathbf{k}^{\prime}$ are shown in the following.

3-(Boc-glycyl)-cis-4-methoxycarbonyl-5-methyloxazolidin-2one (13a): Oil; IR (film): 3355, 2979, 2925, 2852, 1749, 1521, 1393, 1368, 1218, 1164, 1099, 1054, 952, 862, $761 \mathrm{~cm}^{-1}$; ${ }^{1} \mathrm{H} \mathrm{NMR}$ $\left(300 \mathrm{MHz}, \mathrm{CDCl}_{3}\right): \delta 1.42(\mathrm{~d}, J=6.24 \mathrm{~Hz}, 3 \mathrm{H}), 1.45(\mathrm{~s}, 9 \mathrm{H}), 3.81$ $(\mathrm{s}, 3 \mathrm{H}), 4.42-4.50(\mathrm{~m}, 1 \mathrm{H}), 4.57-4.70(\mathrm{~m}, 1 \mathrm{H}), 4.85(\mathrm{~d}, J=$ $8.62 \mathrm{~Hz}, 1 \mathrm{H}), 4.91(\mathrm{dq}, J=6.24,8.62 \mathrm{~Hz}, 1 \mathrm{H}), 4.99-5.13$ (br, $1 \mathrm{H})$. HRMS $\left(\mathrm{FAB}^{+}\right)\left(\mathrm{M}^{+}+1\right)$ : Found: $m / z$ 317.1355. Calcd for $\mathrm{C}_{13} \mathrm{H}_{21} \mathrm{~N}_{2} \mathrm{O}_{7}: 317.1349$.

3-(Boc-alanyl)-cis-4-methoxycarbonyl-5-methyloxazolidin-2one (13b and 13b'): 13b: oil; $[\alpha]_{\mathrm{D}}^{25}+21.08^{\circ}(c 1.28$, EtOH). IR (film): 3391, 2980, 2936, 1794, 1754, 1707, 1507, 1454, 1392, 1367, 1217, 1166, 1099, 1071, 1054, 1020, 981, 953, 862, $758 \mathrm{~cm}^{-1} ;{ }^{1} \mathrm{HNMR}\left(300 \mathrm{MHz}, \mathrm{CDCl}_{3}\right): \delta 1.42(\mathrm{~d}, J=6.24 \mathrm{~Hz}$, $3 \mathrm{H}), 1.43(\mathrm{~d}, J=6.97 \mathrm{~Hz}, 3 \mathrm{H}), 1.43(\mathrm{~s}, 9 \mathrm{H}), 3.80(\mathrm{~s}, 3 \mathrm{H}), 4.90(\mathrm{~d}$, $J=8.80 \mathrm{~Hz}, 1 \mathrm{H}), 4.91(\mathrm{dq}, J=6.24,8.80 \mathrm{~Hz}, 1 \mathrm{H}), 4.98-5.12(\mathrm{br}$, $1 \mathrm{H}), 5.33(\mathrm{dq}, J=6.97,8.07 \mathrm{~Hz}, 1 \mathrm{H}) . \mathrm{HRMS}\left(\mathrm{FAB}^{+}\right)\left(\mathrm{M}^{+}+1\right)$ : Found: $m / z$ 331.1506. Calcd for $\mathrm{C}_{14} \mathrm{H}_{23} \mathrm{~N}_{2} \mathrm{O}_{7}$ : 331.1505. 13b': oil; $[\alpha]_{\mathrm{D}}^{25}-118.63^{\circ}$ (c 1.08, EtOH); IR (film): 3389, 2980, 2936, 1793, $1755,1706,1505,1454,1390,1367,1217,1166,1099,1071$, 1054, 1020, 953, 862, $758 \mathrm{~cm}^{-1} ;{ }^{1} \mathrm{HNMR}\left(300 \mathrm{MHz}, \mathrm{CDCl}_{3}\right): \delta$ $1.42(\mathrm{~d}, J=6.42 \mathrm{~Hz}, 6 \mathrm{H}), 1.44(\mathrm{~s}, 9 \mathrm{H}), 3.81(\mathrm{~s}, 3 \mathrm{H}), 4.80(\mathrm{~d}$, $J=8.44 \mathrm{~Hz}, 1 \mathrm{H}), 4.89(\mathrm{dq}, J=6.42,8.44 \mathrm{~Hz}, 1 \mathrm{H}), 5.20-5.34(\mathrm{br}$, $1 \mathrm{H}), 5.36-5.52(\mathrm{~m}, 1 \mathrm{H})$. HRMS $\left(\mathrm{FAB}^{+}\right)\left(\mathrm{M}^{+}+1\right)$ : Found: $m / z$ 331.1498. Calcd for $\mathrm{C}_{14} \mathrm{H}_{23} \mathrm{~N}_{2} \mathrm{O}_{7}: 331.1505$.

3-(Boc-valyl)-cis-4-methoxycarbonyl-5-methyloxazolidin-2one (13c and 13c'): 13c: $\mathrm{mp} 101.5-102.5^{\circ} \mathrm{C}$ (EtOAc-hexane); $[\alpha]_{\mathrm{D}}^{25}-70.66^{\circ}(c$ 1.17, EtOH); IR (KBr): 3440, 3004, 2973, 2870 , $2840,1795,1760,1718,1698,1509,1458,1366,1212,1164$, $1100,882,795 \mathrm{~cm}^{-1}$; ${ }^{1} \mathrm{HNMR}\left(300 \mathrm{MHz}, \mathrm{CDCl}_{3}\right): \delta 0.83(\mathrm{~d}$, $J=6.79 \mathrm{~Hz}, 3 \mathrm{H}), 1.05(\mathrm{~d}, J=6.79 \mathrm{~Hz}, 3 \mathrm{H}), 1.41(\mathrm{~d}, J=2.93 \mathrm{~Hz}$, $3 \mathrm{H}), 1.45(\mathrm{~s}, 9 \mathrm{H}), 2.30-2.38(\mathrm{~m}, 1 \mathrm{H}), 3.80(\mathrm{~s}, 3 \mathrm{H}), 4.87(\mathrm{dq}$, $J=6.42,8.44 \mathrm{~Hz}, 1 \mathrm{H}), 4.90(\mathrm{~d}, J=8.44 \mathrm{~Hz}, 1 \mathrm{H}), 5.10-5.28(\mathrm{br}$, $1 \mathrm{H}), 5.37(\mathrm{dd}, J=3.67,9.35 \mathrm{~Hz}, 1 \mathrm{H})$. HRMS $\left(\mathrm{FAB}^{+}\right)\left(\mathrm{M}^{+}+1\right)$ : Found: $m / z$ 359.1825. Calcd for $\mathrm{C}_{16} \mathrm{H}_{27} \mathrm{~N}_{2} \mathrm{O}_{7}$ : 359.1818. 13c': oil; $[\alpha]_{\mathrm{D}}^{25}+61.48^{\circ}(c$ 0.96, EtOH); IR (film): 3437, 3392, 2972, 2935, 2875, 1793, 1750, 1705, 1500, 1392, 1365, 1211, 1164, 1098, 1054, 1017, 758, $667 \mathrm{~cm}^{-1}$; ${ }^{1} \mathrm{HNMR}\left(300 \mathrm{MHz}, \mathrm{CDCl}_{3}\right): \delta 0.85$ $(\mathrm{d}, J=6.79 \mathrm{~Hz}, 3 \mathrm{H}), 1.09(\mathrm{~d}, J=6.79 \mathrm{~Hz}, 3 \mathrm{H}), 1.40(\mathrm{~d}$, $J=6.42 \mathrm{~Hz}, 3 \mathrm{H}), 1.44$ (s, 9H), 2.20-2.24 (br, $1 \mathrm{H}), 3.79$ (s, 3H), $4.77(\mathrm{~d}, J=8.44 \mathrm{~Hz}, 1 \mathrm{H}), 4.86(\mathrm{dq}, J=6.42,8.44 \mathrm{~Hz}, 1 \mathrm{H})$, $5.11-5.25(\mathrm{br}, 1 \mathrm{H}), 5.36(\mathrm{dd}, J=3.67,9.35 \mathrm{~Hz}, 1 \mathrm{H})$. HRMS $\left(\mathrm{FAB}^{+}\right)\left(\mathrm{M}^{+}+1\right)$ : Found: $m / z$ 359.1825. Calcd for $\mathrm{C}_{16} \mathrm{H}_{27} \mathrm{~N}_{2} \mathrm{O}_{7}$ : 359.1818 .

3-(Boc-leucyl)-cis-4-methoxycarbonyl-5-methyloxazolidin-2one (13d and 13d'): 13d: $\mathrm{mp} 92.0-93.0^{\circ} \mathrm{C}$ (EtOAc-hexane); $[\alpha]_{\mathrm{D}}^{25}-84.34^{\circ}(c$ 0.86, EtOH); IR (KBr): 3396, 3331, 2963, 2937, 2873, 1796, 1746, 1712, 1524, 1390, 1367, 1253, 1212, 1167, 1100, 1051, 1026, 951, 786, $759 \mathrm{~cm}^{-1} ;{ }^{1} \mathrm{HNMR}(300 \mathrm{MHz}$, $\left.\mathrm{CDCl}_{3}\right): \delta 0.96(\mathrm{~d}, J=6.42 \mathrm{~Hz}, 3 \mathrm{H}), 1.03(\mathrm{~d}, J=6.42 \mathrm{~Hz}, 3 \mathrm{H})$, $1.40(\mathrm{~d}, J=6.42 \mathrm{~Hz}, 3 \mathrm{H}), 1.43(\mathrm{~s}, 9 \mathrm{H}), 1.55-1.90(\mathrm{~m}, 3 \mathrm{H}), 3.80(\mathrm{~s}$, $3 \mathrm{H}), 4.84-4.96(\mathrm{~m}, 2 \mathrm{H}), 4.91(\mathrm{~d}, J=8.80 \mathrm{~Hz}, 1 \mathrm{H}), 5.30-5.52(\mathrm{~m}$, 1H). HRMS $\left(\mathrm{FAB}^{+}\right)\left(\mathrm{M}^{+}+1\right)$ : Found: $m / z$ 373.1972. Calcd for $\mathrm{C}_{17} \mathrm{H}_{29} \mathrm{~N}_{2} \mathrm{O}_{7}$ : 373.1975. 13d': oil; $[\alpha]_{\mathrm{D}}^{25}+31.83^{\circ}$ ( 0 0.31, EtOH); IR (film): 3352, 2960, 2872, 1793, 1756, 1708, 1507, 1456, 1439, 1366, 1212, 1168, 1098, 1051, 1024, 757, $666 \mathrm{~cm}^{-1} ;{ }^{1} \mathrm{HNMR}$ $\left(300 \mathrm{MHz}, \mathrm{CDCl}_{3}\right): \delta 0.93(\mathrm{~d}, J=6.60 \mathrm{~Hz}, 3 \mathrm{H}), 1.03(\mathrm{~d}, J=$ $6.24 \mathrm{~Hz}, 3 \mathrm{H}), 1.42-1.48(\mathrm{~m}, 12 \mathrm{H}), 1.70-1.88(\mathrm{~m}, 3 \mathrm{H}), 3.80(\mathrm{~s}$, $3 \mathrm{H}), 4.77(\mathrm{~d}, J=8.44 \mathrm{~Hz}, 1 \mathrm{H}), 4.86(\mathrm{dq}, J=6.42,8.44 \mathrm{~Hz}$, $1 \mathrm{H}), 5.09(\mathrm{~d}, J=8.49 \mathrm{~Hz}, 1 \mathrm{H}), 5.38-5.56(\mathrm{~m}, 1 \mathrm{H})$. HRMS $\left(\mathrm{FAB}^{+}\right)\left(\mathrm{M}^{+}+1\right)$ : Found: $m / z$ 373.1977. Calcd for $\mathrm{C}_{17} \mathrm{H}_{29} \mathrm{~N}_{2} \mathrm{O}_{7}$ :

\subsection{5.}

3-(Boc-glycyl)-cis-4-methoxycarbonyl-5-(2-phenylethyl)oxazolidin-2-one (13e): Oil; IR (film): 3356, 3062, 3006, 2979, 2933, 1715, 1516, 1438, 1393, 1368, 1215, 1166, 1054, 1030, 754, $701,667 \mathrm{~cm}^{-1} ;{ }^{1} \mathrm{HNMR}\left(300 \mathrm{MHz}, \mathrm{CDCl}_{3}\right): \delta 1.45(\mathrm{~s}, 9 \mathrm{H}), 1.84$ $2.00(\mathrm{~m}, 2 \mathrm{H}), 2.58-2.81(\mathrm{~m}, 1 \mathrm{H}), 2.81-2.96(\mathrm{~m}, 1 \mathrm{H}), 3.79(\mathrm{~s}, 3 \mathrm{H})$, $4.38-4.50(\mathrm{~m}, 1 \mathrm{H}), 4.53-4.64(\mathrm{~m}, 1 \mathrm{H}), 4.66(\mathrm{dt}, J=4.22,8.44 \mathrm{~Hz}$, $1 \mathrm{H}), 4.82(\mathrm{~d}, J=8.44 \mathrm{~Hz}, 1 \mathrm{H}), 5.00-5.16(\mathrm{br}, 1 \mathrm{H}), 7.16-7.38(\mathrm{~m}$, 5H). HRMS $\left(\mathrm{FAB}^{+}\right)\left(\mathrm{M}^{+}+1\right)$ : Found: $m / z$ 407.1814. Calcd for $\mathrm{C}_{20} \mathrm{H}_{27} \mathrm{~N}_{2} \mathrm{O}_{7}$ : 407.1818 .

3-(Boc-alanyl)-cis-4-methoxycarbonyl-5-(2-phenylethyl)oxazolidin-2-one (13f and 13f'): 13f: oil; $[\alpha]_{\mathrm{D}}^{25}-63.78^{\circ}(c 1.08$, EtOH); IR (film): 3626, 3393, 3026, 3006, 2979, 2935, 1825, 1793, 1753, 1709, 1497, 1455, 1369, 1215, 1167, 1073, 1055, 1021, 757, $701 \mathrm{~cm}^{-1}$; ${ }^{1} \mathrm{HNMR}\left(300 \mathrm{MHz}, \mathrm{CDCl}_{3}\right): \delta 1.42(\mathrm{~s}, 9 \mathrm{H}), 1.44(\mathrm{~d}$, $J=6.79 \mathrm{~Hz}, 3 \mathrm{H}), 1.80-2.00(\mathrm{~m}, 2 \mathrm{H}), 2.65-2.85(\mathrm{~m}, 1 \mathrm{H}), 2.85-$ $3.00(\mathrm{~m}, 1 \mathrm{H}), 3.77(\mathrm{~s}, 3 \mathrm{H}), 4.66(\mathrm{ddd}, J=4.04,8.62,9.72 \mathrm{~Hz}, 1 \mathrm{H})$, $4.87(\mathrm{~d}, J=8.62 \mathrm{~Hz}, 1 \mathrm{H}), 4.96-5.10(\mathrm{br}, 1 \mathrm{H}), 5.32(\mathrm{dq}, J=6.79$, $7.15 \mathrm{~Hz}, 1 \mathrm{H}), 7.14-7.38(\mathrm{~m}, 5 \mathrm{H})$. HRMS $\left(\mathrm{FAB}^{+}\right)\left(\mathrm{M}^{+}+1\right)$ : Found: $m / z$ 421.1978. Calcd for $\mathrm{C}_{21} \mathrm{H}_{29} \mathrm{~N}_{2} \mathrm{O}_{7}$ : 421.1975. 13f': oil: $[\alpha]_{\mathrm{D}}^{25}-119.94^{\circ}$ ( $c$ 0.32, EtOH); IR (film): $3384,2979,2955,2933$, 1793, 1752, 1712, 1496, 1454, 1368, 1211, 1168, 1071, 1022, 756, $701 \mathrm{~cm}^{-1}$; ${ }^{1} \mathrm{HNMR}\left(300 \mathrm{MHz}, \mathrm{CDCl}_{3}\right): \delta 1.35(\mathrm{~d}, J=8.83 \mathrm{~Hz}$, $3 \mathrm{H}), 1.36(\mathrm{~s}, 9 \mathrm{H}), 1.82-2.04(\mathrm{~m}, 2 \mathrm{H}), 2.70-2.82(\mathrm{~m}, 1 \mathrm{H}), 2.86-$ $2.98(\mathrm{~m}, 1 \mathrm{H}), 3.78(\mathrm{~s}, 3 \mathrm{H}), 4.61(\mathrm{ddd}, J=4.39,8.29,9.27 \mathrm{~Hz}, 1 \mathrm{H})$, $4.75(\mathrm{~d}, J=8.29 \mathrm{~Hz}, 1 \mathrm{H}), 5.15-5.30(\mathrm{br}, 1 \mathrm{H}), 5.40-5.50(\mathrm{~m}, 1 \mathrm{H})$, 7.15-7.36 (m, 5H). HRMS $\left(\mathrm{FAB}^{+}\right)\left(\mathrm{M}^{+}+1\right)$ : Found: $m / z$ 421.1965. Calcd for $\mathrm{C}_{21} \mathrm{H}_{29} \mathrm{~N}_{2} \mathrm{O}_{7}: 421.1975$.

3-(Boc-valyl)-cis-4-methoxycarbonyl-5-(2-phenylethyl)oxazolidin-2-one (13g and 13g'): 13g: oil; $[\alpha]_{\mathrm{D}}^{25}-53.38^{\circ}(c 0.67$, EtOH); IR (film): 3340, 2968, 2931, 1793, 1753, 1706, 1497, 1456, 1391, 1368, 1211, 1165, 1143, 1016, 756, $701 \mathrm{~cm}^{-1}$; ${ }^{1} \mathrm{H} \mathrm{NMR}\left(300 \mathrm{MHz}, \mathrm{CDCl}_{3}\right): \delta 0.84(\mathrm{~d}, J=6.79 \mathrm{~Hz}, 3 \mathrm{H}), 1.09(\mathrm{~d}$, $J=6.79 \mathrm{~Hz}, 3 \mathrm{H}), 1.44(\mathrm{~s}, 9 \mathrm{H}), 1.86-1.98(\mathrm{~m}, 2 \mathrm{H}), 2.24-2.38(\mathrm{br}$, $1 \mathrm{H}), 2.68-2.82(\mathrm{~m}, 1 \mathrm{H}), 2.84-2.98(\mathrm{~m}, 1 \mathrm{H}), 3.76(\mathrm{~s}, 3 \mathrm{H}), 4.64(\mathrm{dt}$, $J=4.04,8.80 \mathrm{~Hz}, 1 \mathrm{H}), 4.88(\mathrm{~d}, J=8.80 \mathrm{~Hz}, 1 \mathrm{H}), 5.02(\mathrm{~d}, J=$ $9.35 \mathrm{~Hz}, 1 \mathrm{H}), 5.36(\mathrm{dd}, J=3.67,9.35 \mathrm{~Hz}, 1 \mathrm{H}), 7.14-7.34(\mathrm{~m}, 5 \mathrm{H})$. HRMS $\left(\mathrm{FAB}^{+}\right)\left(\mathrm{M}^{+}+1\right)$ : Found: $m / z$ 449.2281. Calcd for $\mathrm{C}_{23} \mathrm{H}_{33} \mathrm{~N}_{2} \mathrm{O}_{7}$ : 449.2288. 13g': oil: $[\alpha]_{\mathrm{D}}^{25}+32.37^{\circ}$ (c 0.50, EtOH); IR (film): 3442, 3395, 3026, 2970, 2932, 2874, 1793, 1753, 1704, 1497, 1391, 1368, 1215, 1164, 1018, 756, $701 \mathrm{~cm}^{-1} ;{ }^{1} \mathrm{HNMR}$ $\left(300 \mathrm{MHz}, \mathrm{CDCl}_{3}\right): \delta 0.80(\mathrm{~d}, J=6.79 \mathrm{~Hz}, 3 \mathrm{H}), 1.04(\mathrm{~d}, J=6.79$ $\mathrm{Hz}, 3 \mathrm{H}), 1.45$ (s, 9H), 1.86-1.98 (m, 2H), 2.06-2.24 (br, 1H), 2.69-2.82 (m, 1H), 2.86-2.98 (m, 1H), $3.78(\mathrm{~s}, 3 \mathrm{H}), 4.59(\mathrm{dt}$, $J=4.77,8.62 \mathrm{~Hz}, 1 \mathrm{H}), 4.73(\mathrm{~d}, J=8.62 \mathrm{~Hz}, 1 \mathrm{H}), 5.17(\mathrm{~d}, J=$ $9.35 \mathrm{~Hz}, 1 \mathrm{H}), 5.44(\mathrm{dd}, J=3.76,9.35 \mathrm{~Hz}, 1 \mathrm{H}), 7.18-7.36(\mathrm{~m}, 5 \mathrm{H})$. HRMS $\left(\mathrm{FAB}^{+}\right)\left(\mathrm{M}^{+}+1\right)$ : Found: $m / z$ 449.2274. Calcd for $\mathrm{C}_{23} \mathrm{H}_{33} \mathrm{~N}_{2} \mathrm{O}_{7}$ : 449.2288 .

3-(Boc-glycyl)-cis-4-methoxycarbonyl-5-phenyloxazolidin-2one (13h): $\mathrm{Mp} 118.0-119.0^{\circ} \mathrm{C}$ (EtOAc-hexane); IR (KBr): 3397 , 3025, 3008, 2978, 2955, 1794, 1742, 1732, 1697, 1523, 1394, $1381,1338,1270,1249,1233,1195,1168,1143,1051,1018,941$, $795,701 \mathrm{~cm}^{-1} ;{ }^{1} \mathrm{H}$ NMR $\left(300 \mathrm{MHz}, \mathrm{CDCl}_{3}\right): \delta 1.46(\mathrm{~s}, 9 \mathrm{H}), 3.21$ (s, 3H), $4.48(\mathrm{dd}, J=4.40,19.26 \mathrm{~Hz}, 1 \mathrm{H}), 4.71(\mathrm{dd}, J=6.79$, $19.26 \mathrm{~Hz}, 1 \mathrm{H}), 5.09$ (d, $J=8.99 \mathrm{~Hz}, 1 \mathrm{H}), 5.04-5.12(\mathrm{br}, 1 \mathrm{H}), 5.82$ $(\mathrm{d}, J=8.99 \mathrm{~Hz}, 1 \mathrm{H}), 7.28-7.46(\mathrm{~m}, 5 \mathrm{H}) . \mathrm{HRMS}\left(\mathrm{FAB}^{+}\right)\left(\mathrm{M}^{+}+\right.$ 1): Found: $m / z$ 379.1516. Calcd for $\mathrm{C}_{18} \mathrm{H}_{23} \mathrm{~N}_{2} \mathrm{O}_{7}: 379.1505$.

3-(Boc-valyl)-cis-4-methoxycarbonyl-5-phenyloxazolidin-2one (13j and $\left.\mathbf{1 3 j}^{\prime}\right)$ : $\mathbf{1 3} \mathbf{j}$ : $\mathrm{mp} 113.5-1114.5^{\circ} \mathrm{C}$ (EtOAc-hexane); $[\alpha]_{\mathrm{D}}^{25}-19.66^{\circ}(c 0.44, \mathrm{EtOH})$; IR (KBr): $3416,2968,1796,1748$, $1710,1510,1456,1370,1343,1217,1163,1134,1053,1020$, 1001, 874, 839, 756, $697 \mathrm{~cm}^{-1} ;{ }^{1} \mathrm{H}$ NMR $\left(300 \mathrm{MHz}, \mathrm{CDCl}_{3}\right): \delta$ 
$0.86(\mathrm{~d}, J=6.97 \mathrm{~Hz}, 3 \mathrm{H}), 1.13(\mathrm{~d}, J=6.79 \mathrm{~Hz}, 3 \mathrm{H}), 1.45(\mathrm{~s}, 9 \mathrm{H})$, $2.28-2.46(\mathrm{br}, 1 \mathrm{H}), 3.18$ (s, 3H), $5.03(\mathrm{~d}, J=9.36 \mathrm{~Hz}, 1 \mathrm{H}), 5.16$ $(\mathrm{d}, J=9.35 \mathrm{~Hz}, 1 \mathrm{H}), 5.44(\mathrm{dd}, J=3.85,9.36 \mathrm{~Hz}, 1 \mathrm{H}), 5.81(\mathrm{~d}$, $J=9.35 \mathrm{~Hz}, 1 \mathrm{H}), 7.65-7.75(\mathrm{~m}, 5 \mathrm{H})$. HRMS $\left(\mathrm{FAB}^{+}\right)\left(\mathrm{M}^{+}+1\right)$ : Found: $m / z$ 421.1989. Calcd for $\mathrm{C}_{21} \mathrm{H}_{29} \mathrm{~N}_{2} \mathrm{O}_{7}: 421.1975$. 13j': $\mathrm{mp}$ $128.5-129.5^{\circ} \mathrm{C}$ (EtOAc-hexane); $[\alpha]_{\mathrm{D}}^{25}-12.52^{\circ}$ ( $c$ 0.54, EtOH); IR (KBr): 3405, 2970, 2934, 1784, 1749, 1699, 1512, 1357, 1277, $1246,1217,1200,1166,1140,1055,1017,891,758,722$, $700 \mathrm{~cm}^{-1} ;{ }^{1} \mathrm{HNMR}\left(300 \mathrm{MHz}, \mathrm{CDCl}_{3}\right): \delta 0.86(\mathrm{~d}, J=6.97 \mathrm{~Hz}$, $3 \mathrm{H}), 1.08(\mathrm{~d}, J=6.79 \mathrm{~Hz}, 3 \mathrm{H}), 1.45(\mathrm{~s}, 9 \mathrm{H}), 2.12-2.32(\mathrm{br}, 1 \mathrm{H})$, $3.23(\mathrm{~s}, 3 \mathrm{H}), 5.02(\mathrm{~d}, J=8.62 \mathrm{~Hz}, 1 \mathrm{H}), 5.22(\mathrm{~d}, J=9.35 \mathrm{~Hz}, 1 \mathrm{H})$, $5.44(\mathrm{dd}, J=3.85,9.35 \mathrm{~Hz}, 1 \mathrm{H}), 5.81(\mathrm{~d}, J=8.62 \mathrm{~Hz}, 1 \mathrm{H}), 7.28$ $7.44(\mathrm{~m}, 5 \mathrm{H})$. Found: $\mathrm{C}, 59.90 ; \mathrm{H}, 6.79 ; \mathrm{N}, 6.65 \%$. Calcd for $\mathrm{C}_{21} \mathrm{H}_{28} \mathrm{~N}_{2} \mathrm{O}_{7}$ : C, 59.98; H, 6.71; N, 6.66\%.

3-(Boc-leucyl)-cis-4-methoxycarbonyl-5-phenyloxazolidin-2one (13k and 13k'): 13k: oil; $[\alpha]_{\mathrm{D}}^{25}-24.29^{\circ}(c 0.63$, EtOH); IR (film): 3350, 2960, 2872, 1793, 1756, 1708, 1507, 1456, 1439, $1366,1212,1168,1098,1051,1025,757 \mathrm{~cm}^{-1} ;{ }^{1} \mathrm{HNMR}(300$ $\left.\mathrm{MHz}, \mathrm{CDCl}_{3}\right): \delta 0.98(\mathrm{~d}, J=6.11 \mathrm{~Hz}, 3 \mathrm{H}), 1.07(\mathrm{~d}, J=6.05 \mathrm{~Hz}$, $3 \mathrm{H}), 1.30-1.42(\mathrm{~m}, 1 \mathrm{H}), 1.45(\mathrm{~s}, 9 \mathrm{H}), 1.72-1.94(\mathrm{~m}, 2 \mathrm{H}), 3.18(\mathrm{~s}$, $3 \mathrm{H}), 4.95(\mathrm{~d}, J=8.44 \mathrm{~Hz}, 1 \mathrm{H}), 5.13(\mathrm{~d}, J=9.17 \mathrm{~Hz}, 1 \mathrm{H}), 5.40$ $5.54(\mathrm{br}, 1 \mathrm{H}), 5.82(\mathrm{~d}, J=9.17 \mathrm{~Hz}, 1 \mathrm{H}), 7.28-7.44(\mathrm{~m}, 5 \mathrm{H})$. HRMS $\left(\mathrm{FAB}^{+}\right)\left(\mathrm{M}^{+}+1\right)$ : Found: $m / z$ 435.2124. Calcd for $\mathrm{C}_{22} \mathrm{H}_{31} \mathrm{~N}_{2} \mathrm{O}_{7}$ : 435.2121. 13k': oil; $[\alpha]_{\mathrm{D}}^{25}-24.49^{\circ}$ ( 0.71 , EtOH); IR (film): 3390, 2980, 2936, 1794, 1754, 1705, 1505, 1454, 1392, $1367,1217,1166,1099,758 \mathrm{~cm}^{-1} ;{ }^{1} \mathrm{HNMR}\left(300 \mathrm{MHz}, \mathrm{CDCl}_{3}\right): \delta$ $0.96(\mathrm{~d}, J=6.60 \mathrm{~Hz}, 3 \mathrm{H}), 1.06(\mathrm{~d}, J=6.60 \mathrm{~Hz}, 3 \mathrm{H}), 1.44(\mathrm{~s}, 9 \mathrm{H})$, $1.38-1.52(\mathrm{~m}, 1 \mathrm{H}), 1.55-1.67(\mathrm{~m}, 1 \mathrm{H}), 1.75-1.92(\mathrm{~m}, 1 \mathrm{H}), 3.24(\mathrm{~s}$, $3 \mathrm{H}), 5.01(\mathrm{~d}, J=8.80 \mathrm{~Hz}, 1 \mathrm{H}), 5.07(\mathrm{~m}, 1 \mathrm{H}), 5.55(\mathrm{ddd}, J=3.49$, $10.3,10.4 \mathrm{~Hz}, 1 \mathrm{H}), 5.80(\mathrm{~d}, J=8.80 \mathrm{~Hz}, 1 \mathrm{H}), 7.28-7.42(\mathrm{~m}, 5 \mathrm{H})$. HRMS $\left(\mathrm{FAB}^{+}\right)\left(\mathrm{M}^{+}+1\right)$ : Found: $m / z$ 435.2135. Calcd for $\mathrm{C}_{22} \mathrm{H}_{31} \mathrm{~N}_{2} \mathrm{O}_{7}$ : 435.2121.

A General Procedure for Conversion of Compounds 13a$13 k^{\prime}$ to the Corresponding Dehydropeptides 14a-14k'. To a solution of $\mathbf{1 3 i}$ ( $70 \mathrm{mg}, 0.18 \mathrm{mmol})$ in dry THF $(3 \mathrm{~mL})$ was added 4.0 molar equivalents of LiHMDS in dry THF $(2 \mathrm{~mL})$ at $-45^{\circ} \mathrm{C}$ under $\mathrm{N}_{2}$ and the solution was stirred for $50 \mathrm{~min}$ at that temperature. After the reaction was quenched with $1 \mathrm{M} \mathrm{AcOH}$ in THF at $-45^{\circ} \mathrm{C}$, the solvent was removed in vacuo to give a residue, which was partitioned between EtOAc and water. The aqueous layer was extracted with EtOAc and the combined extracts were washed with brine, dried oner $\mathrm{MgSO}_{4}$, and concentrated in vacuo. The residual oil was subjected to preparative TLC $\left(\mathrm{SiO}_{2}\right.$, benzene/EtOAc $=$ $4 / 1, \mathrm{v} / \mathrm{v})$ to give methyl $(E)-N$-Boc-alanyldehydrophenylalaninate (14i) in $68 \%(43 \mathrm{mg})$ and methyl $(Z)-N$-Boc-alanyldehydrophenylalaninate $\left(\mathbf{1 4} \mathbf{i}^{\prime}\right)$ in $10 \%(6 \mathrm{mg})$ yields, respectively. $(E)-\mathbf{1 4 i}$ : oil; $[\alpha]_{\mathrm{D}}^{25}-2.10^{\circ}$ (c 0.54, EtOH); IR (film): 3317, 3057, 2979, 2932, $2853,1718,1680,1644,1493,1367,1267,1167,1114,1068$, 1020, 858, 769, 737, $692 \mathrm{~cm}^{-1} ;{ }^{1} \mathrm{HNMR}\left(300 \mathrm{MHz}, \mathrm{CDCl}_{3}\right): \delta$ $1.43(\mathrm{~d}, J=7.15 \mathrm{~Hz}, 3 \mathrm{H}), 1.47$ (s, 9H), $3.64(\mathrm{~s}, 3 \mathrm{H}), 4.22-4.42(\mathrm{br}$, $1 \mathrm{H}), 5.16(\mathrm{~d}, J=7.34 \mathrm{~Hz}, 1 \mathrm{H}), 7.16-7.34(\mathrm{~m}, 5 \mathrm{H}), 7.69(\mathrm{~s}, 1 \mathrm{H})$, 8.38-8.64 (br, 1H). HRMS $\left(\mathrm{FAB}^{+}\right)\left(\mathrm{M}^{+}+1\right)$ : Found: $m / z$ 349.1759. Calcd for $\mathrm{C}_{18} \mathrm{H}_{25} \mathrm{~N}_{2} \mathrm{O}_{5}: 349.1764$. When the olefinic proton was irradiated, $3.9 \% \mathrm{NOE}$ was observed for the amide $\mathrm{NH}$ proton. (Z)-14i': $\mathrm{mp} 116.0-117.0^{\circ} \mathrm{C}$ (EtOAc-hexane); $[\alpha]_{\mathrm{D}}^{25}$ $+66.06^{\circ}$ (c 0.19, EtOH); IR (KBr): 3390, 3370, 3058, 2984, 2949, $1715,1693,1644,1500,1478,1273,1206,1163,1118,1065$, 1010, 771, $694 \mathrm{~cm}^{-1}$; ${ }^{1} \mathrm{HNMR}\left(300 \mathrm{MHz}, \mathrm{CDCl}_{3}\right): \delta 1.42(\mathrm{~d}$, $J=7.08 \mathrm{~Hz}, 3 \mathrm{H}), 1.45(\mathrm{~s}, 9 \mathrm{H}), 3.84(\mathrm{~s}, 3 \mathrm{H}), 4.26-4.38(\mathrm{br}, 1 \mathrm{H})$, 4.92-5.08 (br, 1H), 7.30-7.50 (m, 5H), 7.43 (s, 1H), 7.68-7.80 (br, $1 \mathrm{H})$. Found: C, 61.88; H, 7.00; N, 7.90\%. Calcd for $\mathrm{C}_{18} \mathrm{H}_{24} \mathrm{~N}_{2} \mathrm{O}_{5}$ : C, 62.05; H, 6.94; N, 8.04\%.
In a similar way, compounds $\mathbf{1 3} \mathbf{a}-\mathbf{1 3} \mathbf{h}^{\prime}$ and $\mathbf{1 3} \mathbf{j}-\mathbf{1 3} \mathbf{k}^{\prime}$ were converted into $\mathbf{1 4} \mathbf{a}-\mathbf{1 4} \mathbf{h}^{\prime}$ and $\mathbf{1 4} \mathbf{j}-\mathbf{1 4} \mathbf{k}^{\prime}$, whose physical and spectral data were shown in following.

Methyl 2-(Boc-glycylamino)-2-butenoate (14a and 14a'): (E)-14a: oil; IR (film): 3346, 2979, 2933, 1707, 1518, 1393, 1368, 1252, 1167, 1054, 1031, 950, 861, $757 \mathrm{~cm}^{-1} ;{ }^{1} \mathrm{HNMR}$ $\left(300 \mathrm{MHz}, \mathrm{CDCl}_{3}\right): \delta 1.46(\mathrm{~s}, 9 \mathrm{H}), 2.08(\mathrm{~d}, J=7.52 \mathrm{~Hz}, 3 \mathrm{H})$, $3.82-3.88(\mathrm{~m}, 5 \mathrm{H}), 5.24-5.38(\mathrm{br}, 1 \mathrm{H}), 5.44(\mathrm{q}, J=7.52 \mathrm{~Hz}, 1 \mathrm{H})$, 7.98-8.12 (br, 1H). HRMS $\left(\mathrm{FAB}^{+}\right)\left(\mathrm{M}^{+}+1\right)$ : Found: $m / z$ 273.1444. Calcd for $\mathrm{C}_{12} \mathrm{H}_{21} \mathrm{~N}_{2} \mathrm{O}_{5}$ : 273.1451. When the amide $\mathrm{NH}$ proton was irradiated, $2.0 \% \mathrm{NOE}$ was observed for the olefinic proton. (Z)-14a': oil (Lit. mp $100.0-101.5^{\circ} \mathrm{C},{ }^{3 \mathrm{~h}} 100^{\circ} \mathrm{C},{ }^{3 \mathrm{i}}$ and $103-$ $104^{\circ} \mathrm{C}^{20}$ ). IR (film) $3321,2979,2959,2932,1721,1514,1439$, $1368,1281,1168,1073,1052,1029,949,863,756 \mathrm{~cm}^{-1}$; ${ }^{1} \mathrm{H}$ NMR $\left(300 \mathrm{MHz}, \mathrm{CDCl}_{3}\right): \delta 1.47(\mathrm{~s}, 9 \mathrm{H}), 1.77(\mathrm{~d}, J=7.15 \mathrm{~Hz}, 3 \mathrm{H}), 3.76$ $(\mathrm{s}, 3 \mathrm{H}), 3.92(\mathrm{~d}, J=5.50 \mathrm{~Hz}, 2 \mathrm{H}), 5.24-5.36(\mathrm{br}, 1 \mathrm{H}), 6.84(\mathrm{q}$, $J=7.15 \mathrm{~Hz}, 1 \mathrm{H}), 7.50-7.60(\mathrm{br}, 1 \mathrm{H})$. HRMS $\left(\mathrm{FAB}^{+}\right)\left(\mathrm{M}^{+}+1\right)$ : Found: $m / z$ 273.1455. Calcd for $\mathrm{C}_{12} \mathrm{H}_{21} \mathrm{~N}_{2} \mathrm{O}_{5}: 273.1451$.

Methyl 2-(Boc-alanylamino)-2-butenoate (14b and 14b'): (E)-14b: $\mathrm{mp}$ 96.5-97.0 ${ }^{\circ} \mathrm{C}$ (EtOAc-hexane) (Lit. mp 100$\left.101^{\circ} \mathrm{C}\right) ;^{6 \mathrm{f}}[\alpha]_{\mathrm{D}}^{25}-19.83^{\circ}$ (c 1.10, EtOH); IR (KBr): 3319, 3047, 2982, 2955, 2935, 1738, 1677, 1662, 1531, 1367, 1345, 1254, $1167,1116,1070,1043,1026,858,788,757,715 \mathrm{~cm}^{-1}$; ${ }^{1} \mathrm{HNMR}$ $\left(300 \mathrm{MHz}, \mathrm{CDCl}_{3}\right): \delta 1.38(\mathrm{~d}, J=7.15 \mathrm{~Hz}, 3 \mathrm{H}), 1.45(\mathrm{~s}, 9 \mathrm{H}), 2.06$ (d, $J=8.99 \mathrm{~Hz}, 3 \mathrm{H}), 3.82$ (s, 3H), 4.35-4.60 (br, 1H), 4.90-5.15 (br, 1H), 7.17 (q, $J=8.99 \mathrm{~Hz}, 1 \mathrm{H}), 7.96-8.16$ (br, 1H). HRMS $\left(\mathrm{FAB}^{+}\right)\left(\mathrm{M}^{+}+1\right)$ : Found: $m / z$ 287.1600. Calcd for $\mathrm{C}_{13} \mathrm{H}_{23} \mathrm{~N}_{2} \mathrm{O}_{5}$ : 287.1607. When the olefinic proton was irradiated, $2.0 \%$ NOE was observed for the amide NH proton. (Z)-14b': mp $112.0-113.0^{\circ} \mathrm{C}$ (EtOAc-hexane) (Lit. Mp $114^{\circ} \mathrm{C},{ }^{3 \mathrm{i}} 115-116^{\circ} \mathrm{C},{ }^{6 \mathrm{f}}$ and $116^{\circ} \mathrm{C}^{21}$ ); $[\alpha]_{\mathrm{D}}^{25}-18.66^{\circ}$ (c 0.42, EtOH); IR (KBr): 3336, 3292, 2980, 2948, 1720, 1673, 1524, 1323, 1281, 1166, 1050, 772, 705, $673 \mathrm{~cm}^{-1} ;{ }^{1} \mathrm{H}$ NMR $\left(300 \mathrm{MHz}, \mathrm{CDCl}_{3}\right): \delta 1.42(\mathrm{~d}, J=6.97 \mathrm{~Hz}$, $3 \mathrm{H}), 1.46(\mathrm{~s}, 9 \mathrm{H}), 1.76(\mathrm{~d}, J=7.15 \mathrm{~Hz}, 3 \mathrm{H}), 3.76(\mathrm{~s}, 3 \mathrm{H}), 4.20$ 4.38 (br, 1H), 4.90-5.10 (br, 1H), 6.82 (q, $J=7.15 \mathrm{~Hz}, 1 \mathrm{H}), 7.46-$ 7.58 (br, 1H). HRMS $\left(\mathrm{FAB}^{+}\right)\left(\mathrm{M}^{+}+1\right)$ : Found: $m / z$ 287.1610. Calcd for $\mathrm{C}_{13} \mathrm{H}_{23} \mathrm{~N}_{2} \mathrm{O}_{5}$ : 287.1607 .

Methyl 2-(Boc-valylamino)-2-butenoate (14c and 14c'): (E)14c: $\mathrm{mp} 133.5-134.0^{\circ} \mathrm{C}$ (EtOAc-hexane); $[\alpha]_{\mathrm{D}}^{25}-5.20^{\circ}($ c 1.29, EtOH); IR (KBr): 3330, 3276, 3022, 2976, 2955, 2871, 1735, $1685,1666,1523,1434,1369,1353,1248,1172,1045,1021$, 1000, 930, 880,830,808 $\mathrm{cm}^{-1}$; ${ }^{1} \mathrm{HNMR}\left(300 \mathrm{MHz}, \mathrm{CDCl}_{3}\right): \delta$ $0.93(\mathrm{~d}, J=6.79 \mathrm{~Hz}, 3 \mathrm{H}), 0.98(\mathrm{~d}, J=6.79 \mathrm{~Hz}, 3 \mathrm{H}), 1.45(\mathrm{~s}, 9 \mathrm{H})$, $2.18-2.25(\mathrm{~m}, 1 \mathrm{H}), 2.19(\mathrm{~d}, J=7.52 \mathrm{~Hz}, 3 \mathrm{H}), 3.84(\mathrm{~s}, 3 \mathrm{H}), 3.90$ 4.04 (br, 1H), 4.90-5.12 (br, 1H), 7.20 (q, $J=7.70 \mathrm{~Hz}, 1 \mathrm{H}), 7.75-$ 7.85 (br, $1 \mathrm{H})$. Found: $\mathrm{C}, 57.10 ; \mathrm{H}, 8.35 ; \mathrm{N}, 8.89 \%$. Calcd for $\mathrm{C}_{15} \mathrm{H}_{26} \mathrm{~N}_{2} \mathrm{O}_{5}: \mathrm{C}, 57.31 ; \mathrm{H}, 8.34 ; \mathrm{N}, 8.91 \%$. When the olefinic proton was irradiated, $1.1 \% \mathrm{NOE}$ was observed for the amide NH proton. (Z)-14c': mp 99.5-100.5 ${ }^{\circ} \mathrm{C}$ (EtOAc-hexane); $[\alpha]_{\mathrm{D}}^{25}$ $-11.51^{\circ}$ (c 0.66, EtOH); IR (KBr): 3326, 3284, 2955, 2871, $1732,1685,1660,1525,1508,1300,1278,1247,1169,1070$, $1045,932,876,806 \mathrm{~cm}^{-1}$; ${ }^{1} \mathrm{HNMR}\left(300 \mathrm{MHz}, \mathrm{CDCl}_{3}\right): \delta 0.98(\mathrm{~d}$, $J=6.79 \mathrm{~Hz}, 3 \mathrm{H}), 1.03(\mathrm{~d}, J=6.79 \mathrm{~Hz}, 3 \mathrm{H}), 1.17(\mathrm{~d}, J=7.34 \mathrm{~Hz}$, $3 \mathrm{H}), 1.45(\mathrm{~s}, 9 \mathrm{H}), 2.14-2.32(\mathrm{~m}, 1 \mathrm{H}), 3.76(\mathrm{~s}, 3 \mathrm{H}), 4.00-4.10(\mathrm{~m}$, 1H), 4.96-5.14 (br, 1H), 6.83 (q, $J=7.15 \mathrm{~Hz}, 1 \mathrm{H}), 7.26-7.35$ (br, $1 \mathrm{H})$. Found: $\mathrm{C}, 57.31 ; \mathrm{H}, 8.45 ; \mathrm{N}, 8.87 \%$. Calcd for $\mathrm{C}_{15} \mathrm{H}_{26} \mathrm{~N}_{2} \mathrm{O}_{5}$. C, $57.31 ; \mathrm{H}, 8.34 ; \mathrm{N}, 8.91 \%$.

Methyl 2-(Boc-leucylamino)-2-butenoate (14d and 14d'): (E)-14d: mp 93.5-94.5 ${ }^{\circ} \mathrm{C}$ (EtOAc-hexane); $[\alpha]_{\mathrm{D}}^{25}-20.08^{\circ}(c$ 0.91, EtOH); IR (KBr): 3281, 3052, 2976, 2925, 2872, 1727, 1664, 1536, 1366, 1352, 1292, 1257, 1166, 1047, 1023, 850, $785 \mathrm{~cm}^{-1}$; ${ }^{1} \mathrm{HNMR}\left(300 \mathrm{MHz}, \mathrm{CDCl}_{3}\right): \delta 0.94(\mathrm{~d}, J=6.23 \mathrm{~Hz}, 3 \mathrm{H}), 0.96(\mathrm{~d}$, 
$J=6.42 \mathrm{~Hz}, 3 \mathrm{H}), 1.45(\mathrm{~s}, 9 \mathrm{H}), 1.46-1.52(\mathrm{~m}, 1 \mathrm{H}), 1.66-1.78(\mathrm{~m}$, 2H), 2.09 (d, $J=7.70 \mathrm{~Hz}, 3 \mathrm{H}), 3.83(\mathrm{~s}, 3 \mathrm{H}), 4.06-4.22(\mathrm{br}, 1 \mathrm{H})$, 4.76-4.96 (br, 1H), 7.20 (q, $J=7.70 \mathrm{~Hz}, 1 \mathrm{H}), 7.90-8.10$ (br, $1 \mathrm{H})$. HRMS $\left(\mathrm{FAB}^{+}\right)\left(\mathrm{M}^{+}+1\right)$ : Found: $m / z$ 329.1984. Calcd for $\mathrm{C}_{16} \mathrm{H}_{29} \mathrm{~N}_{2} \mathrm{O}_{5}$ : 329.2077. When the olefinic proton was irradiated, $2.1 \%$ NOE was observed for the amide NH proton. $(Z)-\mathbf{1 4} \mathbf{d}^{\prime}$ : oil: $[\alpha]_{\mathrm{D}}^{25}+71.63^{\circ}(c$ 0.20, EtOH); IR (neat): $3348,2979,2932,2853$, $1710,1521,1454,1393,1368,1280,1252,1167,1054,950,861$, $760 \mathrm{~cm}^{-1} ;{ }^{1} \mathrm{HNMR}\left(300 \mathrm{MHz}, \mathrm{CDCl}_{3}\right): \delta 0.95(\mathrm{~d}, J=6.05 \mathrm{~Hz}$, $3 \mathrm{H}), 0.96(\mathrm{~d}, J=6.41 \mathrm{~Hz}, 3 \mathrm{H}), 1.46(\mathrm{~s}, 9 \mathrm{H}), 1.55-1.80(\mathrm{~m}, 6 \mathrm{H})$, $3.76(\mathrm{~s}, 3 \mathrm{H}), 4.05-4.30(\mathrm{br}, 1 \mathrm{H}), 4.80-5.00(\mathrm{br}, 1 \mathrm{H}), 6.81$ (q, $J=7.15 \mathrm{~Hz}, 1 \mathrm{H}), 7.46-7.58(\mathrm{br}, 1 \mathrm{H})$. HRMS $\left(\mathrm{FAB}^{+}\right)\left(\mathrm{M}^{+}+1\right)$ : Found: $m / z$ 329.2079. Calcd for $\mathrm{C}_{16} \mathrm{H}_{29} \mathrm{~N}_{2} \mathrm{O}_{5}: 329.2077$.

Methyl 2-(Boc-glycylamino)-5-phenyl-2-pentenoate (14e and 14e'): (E)-14e: oil; IR (film): 3326, 2978, 2928, 2849, 1715, $1681,1520,1455,1367,1249,1165,1066,751,700 \mathrm{~cm}^{-1}$; ${ }^{1} \mathrm{HNMR}\left(300 \mathrm{MHz}, \mathrm{CDCl}_{3}\right): \delta 1.46(\mathrm{~s}, 9 \mathrm{H}), 2.77(\mathrm{t}, J=7.34 \mathrm{~Hz}$, $2 \mathrm{H}), 2.82-2.93(\mathrm{~m}, 2 \mathrm{H}), 3.80(\mathrm{~s}, 3 \mathrm{H}), 3.86(\mathrm{~d}, J=5.87 \mathrm{~Hz}, 2 \mathrm{H})$, 5.10-5.24 (br, 1H), 7.16-7.34 (m, 6H), 8.00-8.10 (br, 1H). HRMS $\left(\mathrm{FAB}^{+}\right)\left(\mathrm{M}^{+}+1\right)$ : Found: $m / z$ 363.1929. Calcd for $\mathrm{C}_{19} \mathrm{H}_{27} \mathrm{~N}_{2} \mathrm{O}_{5}$ : 363.1920. When the methylene protons at $\gamma$-protons were irradiated, $1.1 \%$ NOE was observed for the methyl ester protons. $(Z)$ 14e': oil; IR (film): 3316, 2979, 2933, 1712, 1518, 1454, 1367, 1248, 1167, 1066, 859, 755, $700 \mathrm{~cm}^{-1}$; ${ }^{1} \mathrm{HNMR} \quad(300 \mathrm{MHz}$, $\left.\mathrm{CDCl}_{3}\right): \delta 1.45(\mathrm{~s}, 9 \mathrm{H}), 2.47(\mathrm{dt}, J=7.32,7.56 \mathrm{~Hz}, 2 \mathrm{H}), 2.78(\mathrm{t}$, $J=7.56 \mathrm{~Hz}, 2 \mathrm{H}), 3.75(\mathrm{~s}, 3 \mathrm{H}), 3.87(\mathrm{~d}, J=5.87 \mathrm{~Hz}, 2 \mathrm{H}), 5.09-$ 5.22 (br, 1H), 6.73 (t, $J=7.32 \mathrm{~Hz}, 1 \mathrm{H}), 7.15-7.35(\mathrm{~m}, 6 \mathrm{H})$. HRMS $\left(\mathrm{FAB}^{+}\right)\left(\mathrm{M}^{+}+1\right)$ : Found: $m / z$ 363.1920. Calcd for $\mathrm{C}_{19} \mathrm{H}_{27} \mathrm{~N}_{2} \mathrm{O}_{5}$ : 363.1920 .

Methyl 2-(Boc-alanylamino)-5-phenyl-2-pentenoate (14f and 14f'): (E)-14f: oil; $[\alpha]_{\mathrm{D}}^{25}-22.10^{\circ}$ (c 0.17, EtOH): IR (film): 3311 , 2978, 2932, 1684, 1605, 1521, 1454, 1366, 1249, 1167, 1066, 1021, 749, $699 \mathrm{~cm}^{-1}$; ${ }^{1} \mathrm{HNMR}\left(300 \mathrm{MHz}, \mathrm{CDCl}_{3}\right): \delta 1.38(\mathrm{~d}$, $J=7.15 \mathrm{~Hz}, 3 \mathrm{H}), 1.45(\mathrm{~s}, 9 \mathrm{H}), 2.72-2.84(\mathrm{~m}, 2 \mathrm{H}), 2.84-2.96(\mathrm{~m}$, 2H), 3.80 (s, 3H), 4.14-4.28 (br, 1H), 4.90-5.08 (br, 1H), 7.14$7.32(\mathrm{~m}, 6 \mathrm{H}), 8.06-8.16(\mathrm{br}, 1 \mathrm{H})$. HRMS $\left(\mathrm{FAB}^{+}\right)\left(\mathrm{M}^{+}+1\right)$ : Found: $m / z$ 377.2082. Calcd for $\mathrm{C}_{20} \mathrm{H}_{29} \mathrm{~N}_{2} \mathrm{O}_{5}: 377.2077$. (Z)-14f': oil; $[\alpha]_{\mathrm{D}}^{25}-1.95^{\circ}$ (c 0.44, EtOH); IR (film): 3299, 2979, 2931, 1790, 1684, 1650, 1498, 1366, 1269, 1249, 1164, 1065, 1023, 746, $699 \mathrm{~cm}^{-1} ;{ }^{1} \mathrm{HNMR}\left(300 \mathrm{MHz}, \mathrm{CDCl}_{3}\right): \delta 1.38(\mathrm{~d}, J=7.15 \mathrm{~Hz}$, $3 \mathrm{H}), 1.44(\mathrm{~s}, 9 \mathrm{H}), 2.46(\mathrm{q}, J=7.52 \mathrm{~Hz}, 2 \mathrm{H}), 2.78(\mathrm{t}, J=7.52 \mathrm{~Hz}$, $2 \mathrm{H}), 3.75$ (s, 3H), 4.16-4.30 (br, 1H), 4.89-4.96 (br, 1H), $6.71(\mathrm{t}$, $J=7.33 \mathrm{~Hz}, 1 \mathrm{H}), 7.16-7.36(\mathrm{~m}, 6 \mathrm{H})$. HRMS $\left(\mathrm{FAB}^{+}\right)\left(\mathrm{M}^{+}+1\right)$ : Found: $m / z$ 377.2088. Calcd for $\mathrm{C}_{20} \mathrm{H}_{29} \mathrm{~N}_{2} \mathrm{O}_{5}$ : 377.2077 .

Methyl 2-(Boc-valylamino)-5-phenyl-2-pentenoate (14g and 14g'): (E)-14g: mp 114.0-115.0 ${ }^{\circ} \mathrm{C}$ (EtOAc-hexane); $[\alpha]_{\mathrm{D}}^{25}$ $+3.55^{\circ}$ ( c 0.22, EtOH); IR (KBr): 3329, 3298, 3031, 2981, 2964, 2932, 2873, 1729, 1687, 1666, 1652, 1603, 1526, 1518, 1386, 1300, 1246, 1174, 1010, 744, $698 \mathrm{~cm}^{-1}$; ${ }^{1} \mathrm{HNMR}(300 \mathrm{MHz}$, $\left.\mathrm{CDCl}_{3}\right): \delta 0.93(\mathrm{~d}, J=6.79 \mathrm{~Hz}, 3 \mathrm{H}), 0.99(\mathrm{~d}, J=6.79 \mathrm{~Hz}, 3 \mathrm{H})$, 1.45 (s, 9H), 2.14-2.25 (m, 1H), 2.73-2.84 (m, 2H), 2.81-2.93 (m, 2H), 3.79 (s, 3H), 3.90-4.06 (br, 1H), 4.98-5.14 (br, 1H), 7.14 (t, $J=7.70 \mathrm{~Hz}, 1 \mathrm{H}), 7.18-7.32(\mathrm{~m}, 5 \mathrm{H}), 7.88$ (br s, 1H). HRMS $\left(\mathrm{FAB}^{+}\right)\left(\mathrm{M}^{+}+1\right)$ : Found: $m / z$ 405.2387. Calcd for $\mathrm{C}_{22} \mathrm{H}_{33} \mathrm{~N}_{2} \mathrm{O}_{5}$ : 405.2390. When the $\gamma$-methylene protons were irradiated, $1.7 \%$ NOE was observed for protons of the methyl ester. (Z)-149': mp 99.0-100.0 ${ }^{\circ} \mathrm{C}$ (EtOAc-hexane); $[\alpha]_{\mathrm{D}}^{25}+14.86^{\circ}(c 0.07$, EtOH); IR (KBr): 3331, 3293, 3018, 2965, 2839, 1732, 1723, 1685, 1662, 1524, 1507, 1294, 1169, 750, $699 \mathrm{~cm}^{-1}$; ${ }^{1} \mathrm{HNMR}(300 \mathrm{MHz}$, $\left.\mathrm{CDCl}_{3}\right): \delta 0.94(\mathrm{~d}, J=6.79 \mathrm{~Hz}, 3 \mathrm{H}), 1.00(\mathrm{~d}, J=6.79 \mathrm{~Hz}, 3 \mathrm{H})$, $1.43(\mathrm{~s}, 9 \mathrm{H}), 2.15-2.24(\mathrm{~m}, 1 \mathrm{H}), 2.48(\mathrm{q}, J=7.34 \mathrm{~Hz}, 2 \mathrm{H}), 2.79(\mathrm{t}$, $J=7.34 \mathrm{~Hz}, 2 \mathrm{H}), 3.75(\mathrm{~s}, 3 \mathrm{H}), 3.97(\mathrm{dd}, J=6.05,8.07 \mathrm{~Hz}, 1 \mathrm{H})$,
4.92-5.10 (br, 1H), $6.72(\mathrm{t}, J=7.34 \mathrm{~Hz}, 1 \mathrm{H}), 7.06$ (br s, 1H) 7.16$7.34(\mathrm{~m}, 5 \mathrm{H})$. HRMS $\left(\mathrm{FAB}^{+}\right)\left(\mathrm{M}^{+}+1\right)$ : Found: $\mathrm{m} / z$ 405.2396. Calcd for $\mathrm{C}_{22} \mathrm{H}_{33} \mathrm{~N}_{2} \mathrm{O}_{5}: 405.2390$.

Methyl $N$-Boc-glycyldehydrophenylalaninate $(14 \mathrm{~h}$ and 14h'): (E)-14h: mp 130.5-131.5 ${ }^{\circ} \mathrm{C}$ (EtOAc-hexane); IR (KBr): 3286, 3240, 2979, 2940, 1747, 1698, 1636, 1539, 1439, 1364, 1299, 1252, 1219, 1166, 982, 942, 760, $705 \mathrm{~cm}^{-1}$; ${ }^{1} \mathrm{HNMR}(300$ $\left.\mathrm{MHz}, \mathrm{CDCl}_{3}\right): \delta 1.48(\mathrm{~s}, 9 \mathrm{H}), 3.64(\mathrm{~s}, 3 \mathrm{H}), 3.92(\mathrm{~d}, J=5.87 \mathrm{~Hz}$, $2 \mathrm{H}), 5.34(\mathrm{t}, J=5.89 \mathrm{~Hz}, 1 \mathrm{H}), 7.20-7.36(\mathrm{~m}, 5 \mathrm{H}), 7.81(\mathrm{~s}, 1 \mathrm{H})$, 8.37 (br s, 1H). Found: C, 60.82; H, 6.59; N, 8.36\%. Calcd for $\mathrm{C}_{17} \mathrm{H}_{22} \mathrm{~N}_{2} \mathrm{O}_{5}: \mathrm{C}, 61.06 ; \mathrm{H}, 6.63 ; \mathrm{N}, 8.38 \%$. When the amide $\mathrm{NH}$ proton was irradiated, $6.6 \%$ NOE was observed for the olefinic proton. (Z)-14h': oil (Lit. mp $62.5-64^{\circ} \mathrm{C}$ ); ${ }^{3 \mathrm{~h}} \mathrm{IR}$ (film): 3299, 2979, 2951, 2933, 1686, 1638, 1521, 1367, 1252, 1165, 1052, 1030, 865, 754, $697 \mathrm{~cm}^{-1} ;{ }^{1} \mathrm{HNMR}\left(300 \mathrm{MHz}, \mathrm{CDCl}_{3}\right): \delta$ $1.45(\mathrm{~s}, 9 \mathrm{H}), 3.84(\mathrm{~s}, 3 \mathrm{H}), 3.92(\mathrm{~d}, J=5.14 \mathrm{~Hz}, 2 \mathrm{H}), 5.16-5.28$ (br, 1H), 7.31-7.52 (m, 5H), 7.44 (s, 1H), 7.69 (br s, 1H). HRMS $\left(\mathrm{FAB}^{+}\right)\left(\mathrm{M}^{+}+1\right)$ : Found: $m / z$ 335.1614. Calcd for $\mathrm{C}_{17} \mathrm{H}_{23} \mathrm{~N}_{2} \mathrm{O}_{5}$ : 335.1607.

Methyl $N$-Boc-valyldehydrophenylalaninate $\left(14 \mathbf{j}\right.$ and $\left.14 \mathbf{j}^{\prime}\right)$ : (E)-14j: mp 143.5-144.5 ${ }^{\circ} \mathrm{C}$ (EtOAc-hexane); $[\alpha]_{\mathrm{D}}^{25}+22.81^{\circ}(c$ 1.11, EtOH); IR (KBr): 3287, 3050, 3040, 2972, 2880, 1734, 1678, 1661, 1532, 1392, 1367, 1299, 1252, 1222, 1167, 1045, 1021, 750, $697 \mathrm{~cm}^{-1}$; ${ }^{1} \mathrm{HNMR}\left(300 \mathrm{MHz}, \mathrm{CDCl}_{3}\right): \delta 0.98(\mathrm{~d}, J=6.83 \mathrm{~Hz}$, $3 \mathrm{H}), 1.03(\mathrm{~d}, J=6.59 \mathrm{~Hz}, 3 \mathrm{H}), 1.45(\mathrm{~s}, 9 \mathrm{H}), 2.18-2.32(\mathrm{~m}, 1 \mathrm{H})$, 3.64 (s, 3H), 3.98-4.14 (br, 1H), 4.96-5.18 (br, 1H), 7.20-7.34 $(\mathrm{m}, 5 \mathrm{H}), 7.76(\mathrm{~s}, 1 \mathrm{H}), 8.16$ (br s, 1H). HRMS $\left(\mathrm{FAB}^{+}\right)\left(\mathrm{M}^{+}+1\right)$ : Found: $m / z$ 377.2068. Calcd for $\mathrm{C}_{20} \mathrm{H}_{29} \mathrm{~N}_{2} \mathrm{O}_{5}: 377.2077$. When the olefinic proton was irradiated, $2.5 \%$ NOE was observed for the amide $\mathrm{NH}$ proton. (Z)-14j': mp $146.0-147.0^{\circ} \mathrm{C}$ (EtOAc-hexane); $[\alpha]_{\mathrm{D}}^{25}+45.37^{\circ}(c$ 0.15, EtOH); IR (KBr): 3323, 3247, 3010, 2959, $2930,2879,1729,1687,1665,1526,1366,1300,1275,1249$, 1202, 1172, 762, $681 \mathrm{~cm}^{-1} ;{ }^{1} \mathrm{H}$ NMR $\left(300 \mathrm{MHz}, \mathrm{CDCl}_{3}\right): \delta 0.97$ $(\mathrm{d}, J=6.79 \mathrm{~Hz}, 3 \mathrm{H}), 1.03(\mathrm{~d}, J=6.79 \mathrm{~Hz}, 3 \mathrm{H}), 1.46(\mathrm{~s}, 9 \mathrm{H}), 2.18$ $2.34(\mathrm{~m}, 1 \mathrm{H}), 3.84(\mathrm{~s}, 3 \mathrm{H}), 4.05(\mathrm{dd}, J=5.87,8.62 \mathrm{~Hz}, 1 \mathrm{H})$, 4.92-5.10 (br, 1H), 7.30-7.38 (m, 3H), $7.40(\mathrm{~s}, 1 \mathrm{H}), 7.48(\mathrm{~d}$, $J=6.42 \mathrm{~Hz}, 2 \mathrm{H}), 7.54$ (br s, 1H). Found: C, 63.77; H, 7.58; N, 7.28\%. Calcd for $\mathrm{C}_{20} \mathrm{H}_{28} \mathrm{~N}_{2} \mathrm{O}_{5}: \mathrm{C}, 63.81 ; \mathrm{H}, 7.50 ; \mathrm{N}, 7.44 \%$.

Methyl $N$-Boc-leucyldehydrophenylalaninate $(14 \mathrm{k}$ and 14k'): (E)-14k: mp 151.5-152.5 ${ }^{\circ} \mathrm{C}$ (EtOAc-hexane); $[\alpha]_{\mathrm{D}}^{25}$ $+13.71^{\circ}$ (c 0.18, EtOH); IR (KBr): 3276, 3051, 2953, 2870, 1738, $1670,1636,1541,1436,1397,1368,1318,1297,1254,1221$, 1171, 1052, 1030, 982, 758, $696 \mathrm{~cm}^{-1}$; ${ }^{1} \mathrm{HNMR}(300 \mathrm{MHz}$, $\left.\mathrm{CDCl}_{3}\right): \delta 0.96(\mathrm{~d}, J=6.05 \mathrm{~Hz}, 3 \mathrm{H}), 0.98(\mathrm{~d}, J=6.41 \mathrm{~Hz}, 3 \mathrm{H})$, $1.47(\mathrm{~s}, 9 \mathrm{H}), 1.49-1.62(\mathrm{~m}, 1 \mathrm{H}), 1.67-1.84(\mathrm{~m}, 2 \mathrm{H}), 3.64(\mathrm{~s}, 3 \mathrm{H})$, 4.14-4.32 (br, 1H), 4.90 (d, $J=7.32 \mathrm{~Hz}, 1 \mathrm{H}), 7.20-7.34(\mathrm{~m}, 5 \mathrm{H})$, $7.79(\mathrm{~s}, 1 \mathrm{H}), 8.35$ (br s, 1H). HRMS $\left(\mathrm{FAB}^{+}\right)\left(\mathrm{M}^{+}+1\right)$ : Found: $m /$ $z$ 391.2231. Calcd for $\mathrm{C}_{21} \mathrm{H}_{31} \mathrm{~N}_{2} \mathrm{O}_{5}: 391.2233$. When the olefinic proton was irradiated, $4.0 \%$ NOE was observed for the amide $\mathrm{NH}$ proton. (Z)-14k': oil; $[\alpha]_{\mathrm{D}}^{25}+10.31^{\circ}$ (c 0.30, EtOH); IR (film): 3299, 2957, 2940, 2870, 1690, 1512, 1267, 1168, 918, 732, $692 \mathrm{~cm}^{-1} ;{ }^{1} \mathrm{HNMR}\left(300 \mathrm{MHz}, \mathrm{CDCl}_{3}\right): \delta 0.94(\mathrm{~d}, J=6.10 \mathrm{~Hz}$, $3 \mathrm{H}), 0.97(\mathrm{~d}, J=6.34 \mathrm{~Hz}, 3 \mathrm{H}), 1.46(\mathrm{~s}, 9 \mathrm{H}), 1.48-1.52(\mathrm{~m}, 1 \mathrm{H})$, $1.67-1.84(\mathrm{~m}, 2 \mathrm{H}), 3.82(\mathrm{~s}, 3 \mathrm{H}), 4.18-4.32(\mathrm{br}, 1 \mathrm{H}), 4.92(\mathrm{~d}$, $J=8.05 \mathrm{~Hz}, 1 \mathrm{H}), 7.26-7.38(\mathrm{~m}, 3 \mathrm{H}), 7.40(\mathrm{~s}, 1 \mathrm{H}), 7.48(\mathrm{~d}$, $J=7.07 \mathrm{~Hz}, 2 \mathrm{H}), 7.77$ (br s, 1H). HRMS $\left(\mathrm{FAB}^{+}\right)\left(\mathrm{M}^{+}+1\right)$ : Found: $m / z$, 391.2236. Calcd for $\mathrm{C}_{21} \mathrm{H}_{31} \mathrm{~N}_{2} \mathrm{O}_{5}$ : 391.2233 .

4-(N-Z-amino)-1-butyl Acetate (15). To a mixture of 4amino-1-butanol (1.782 g, $20 \mathrm{mmol})$ and sodium carbonate $(2.332 \mathrm{~g}, 22 \mathrm{mmol})$ in water $(46 \mathrm{~mL})$ was added dropwise a solution of $\mathrm{Z}-\mathrm{Cl}(3.410 \mathrm{~g}, 20 \mathrm{mmol})$ in THF $(40 \mathrm{~mL})$ at $0{ }^{\circ} \mathrm{C}$. The mixture was allowed to stand overnight at room temperature and 
the THF was removed in vauo to give a residue, which was partitioned between EtOAc and water. The EtOAc solution was washed with brine, dried, and evaporated in vacuo to afford the product in quantitative yield $(4.46 \mathrm{~g})$.

To a solution of the resulting alcohol $(4.46 \mathrm{~g}, 20 \mathrm{mmol})$ in THF $(20 \mathrm{~mL})$ was added a catalytic amount of DMAP followed by the addition of acetic anhydride $(3.77 \mathrm{~mL}, 40 \mathrm{mmol})$ at room temperature. After stirring for $20 \mathrm{~min}$, the reaction was quenched with a saturated solution of aqueous $\mathrm{NaHCO}_{3}$ and the solvent was removed in vacuo. The residue was dissolved in EtOAc and the organic solution was washed with brine, dried over $\mathrm{MgSO}_{4}$, and evaporated under reduced pressure to afford the desired product 15 in $94 \%$ yield $(4.98 \mathrm{~g})$. Oil; IR (neat): 3344, 3033, 2951, 1723, $1603,1530,1455,1366,1247,1138,1038,993,741,698 \mathrm{~cm}^{-1}$; ${ }^{1} \mathrm{HNMR}\left(300 \mathrm{MHz}, \mathrm{CDCl}_{3}\right): \delta 1.53-1.66(\mathrm{~m}, 4 \mathrm{H}), 2.02(\mathrm{~s}, 3 \mathrm{H})$, $3.17-3.23(\mathrm{~m}, 2 \mathrm{H}), 4.03-4.14(\mathrm{~m}, 2 \mathrm{H}), 5.07$ (s, 2H), 5.08 (br, 1H), 7.33 (br, 5H). HRMS $\left(\mathrm{FAB}^{+}\right)\left(\mathrm{M}^{+}+1\right)$ : Found: $m / z 266.1393$. Calcd for $\mathrm{C}_{14} \mathrm{H}_{20} \mathrm{NO}_{4}$ : 266.1392.

4-(N-Boc- $\mathrm{N}$-Z-amino)-1-butyl Acetate (16). To a solution of $15(4.98 \mathrm{~g}, 18.7 \mathrm{mmol})$ in dry THF $(15 \mathrm{~mL})$ was added a solution of a catalytic amount of DMAP in dry THF $(5 \mathrm{~mL})$ at $0{ }^{\circ} \mathrm{C}$ followed by the addition of a solution of $\mathrm{Boc}_{2} \mathrm{O}(6.22 \mathrm{~g}$, $28.5 \mathrm{mmol}$ ) in dry THF at that temperature. The solution was allowed to stand overnight at room temperature. After evaporation of the solvent, the residue was partitioned between EtOAc and water. The organic solution was washed with brine, dried over $\mathrm{MgSO}_{4}$, and concentrated under reduced pressure to give compound 16 in $81 \%$ yield $(5.58 \mathrm{~g})$. Oil; IR (neat): 2978, 1792, 1739, $1697,1609,1587,1550,1498,1475,1369,1290,1240,1202$, $1100,1072,852,780,753,700 \mathrm{~cm}^{-1} ;{ }^{1} \mathrm{HNMR}\left(300 \mathrm{MHz}, \mathrm{CDCl}_{3}\right)$ : $\delta 1.47(\mathrm{~s}, 9 \mathrm{H}), 1.61-1.66(\mathrm{br}, 4 \mathrm{H}), 2.02(\mathrm{~s}, 3 \mathrm{H}), 3.67(\mathrm{t}, J=$ $6.83 \mathrm{~Hz}, 2 \mathrm{H}), 4.04(\mathrm{t}, J=6.10 \mathrm{~Hz}, 2 \mathrm{H}), 5.22(\mathrm{~s}, 2 \mathrm{H}), 7.32-7.40$ $(\mathrm{m}, 5 \mathrm{H})$. HRMS $\left(\mathrm{FAB}^{+}\right)\left(\mathrm{M}^{+}+1\right)$ : Found: $m / z$ 366.1916. Calcd for $\mathrm{C}_{19} \mathrm{H}_{28} \mathrm{NO}_{6}$ : 366.1916 .

4-(N-Boc- $\mathrm{N}$-Z-amino)-1-butanol (17). Compound 16 $(1.120 \mathrm{~g}, 3.07 \mathrm{mmol})$ was dissolved in $0.5 \mathrm{M} \mathrm{HCl}$ in $\mathrm{MeOH}$ $(63 \mathrm{~mL})$ at $0{ }^{\circ} \mathrm{C}$. The solution was gradually warmed to room temperature and allowed to stand overnight. After neutralization with a saturatated solution of $\mathrm{NaHCO}_{3}$ at $0{ }^{\circ} \mathrm{C}$, the $\mathrm{MeOH}$ was removed in vacuo to afford a residue, which was extracted with EtOAc. The EtOAc solution was washed with brine, dried over $\mathrm{MgSO}_{4}$, and evaporated in vacuo to give a residue, which was subjected to silica gel column chromatography (eluent; hexane/ EtOAc $=1 / 1, \mathrm{v} / \mathrm{v})$ giving the product $17(765 \mathrm{mg}, 77 \%)$. Oil; IR (neat): 3487, 2977, 2938, 2870, 1782, 1736, 1455, 1369, 1294, $1253,1119,1065,1030,751 \mathrm{~cm}^{-1} ;{ }^{1} \mathrm{HNMR}\left(300 \mathrm{MHz}, \mathrm{CDCl}_{3}\right): \delta$ 1.47 (s, 9H), 1.51-1.68 (m, 4H), 2.02 (br, 1H), 3.59-3.69 (m, 4H), $5.21(\mathrm{~s}, 2 \mathrm{H}), 7.31-7.38(\mathrm{~m}, 5 \mathrm{H})$. HRMS $\left(\mathrm{FAB}^{+}\right)\left(\mathrm{M}^{+}+1\right)$ : Found: $m / z$ 324.1811. Calcd for $\mathrm{C}_{17} \mathrm{H}_{26} \mathrm{NO}_{5}$ : 324.1811 .

Isomerization of the $(E)-N$-Boc- $\alpha, \beta$-didehydroamino Acid Methyl Ester 1 to the Corresponding $Z$ Isomer $1^{\prime}$ by Iodine. To a solution of $1 \mathbf{a}(41 \mathrm{mg}, 0.19 \mathrm{mmol})$ in dry THF $(2 \mathrm{~mL})$ was added a catalytic amount of iodine at room temperature under $\mathrm{N}_{2}$. The solution was stirred for $19 \mathrm{~h}$ at that temperature and quenched with a saturated solution of $\mathrm{NaHSO}_{3}$. The solvent was removed in vacuo to afford the residue, which was extracted with EtOAc. The organic solution was washed with brine, dried over $\mathrm{MgSO}_{4}$, and concentrated in vacuo to give a residue, which was subjected to preparative TLC $\left(\mathrm{SiO}_{2}\right.$, hexane/EtOAc $\left.=4 / 1, \mathrm{v} / \mathrm{v}\right)$ to afford $\mathrm{Z}$

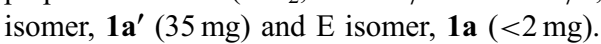

Compounds $\mathbf{1 b}-\mathbf{1 h}$ were isomerized to their $\mathrm{Z}$ isomers in the same way. The results are summarized in Table 7 .

\section{References}

1 a) T. Ueno, T. Nakashima, Y. Hayashi, H. Fukami, Agric. Biol. Chem. 1975, 39, 1115. b) R. D. Durbin, Toxins in Plant Disease, Physiological Ecology Series, Academic Press, 1981, p. 357 . c) T. Shiroza, N. Ebisawa, A. Kojima, K. Furihata, A. Shimazu, T. Endo, H. Seto, N. Otake, Agric. Biol. Chem. 1982, 46, 1885. d) D. P. Botes, A. A. Tuinman, P. L. Wessels, C. C. Viljoen, H. Kruger, D. H. Williams, S. Santikarn, R. J. Smith, S. J. Hammond, J. Chem. Soc., Perkin Trans. 1 1984, 2311. e) M. F. Mackay, A. Van Donkelaar, C. C. J. Culvenor, J. Chem. Soc., Chem. Commun. 1986, 1219. f) K. Nagaoka, M. Matsumoto, J. Oono, K. Yokoi, S. Ishizeki, T. Nakashima, J. Antibiot. 1986, 36, 1527. g) K. L. Rinehart, K. Harada, M. Namikoshi, C. Chen, C. A. Harvis, M. H. G. Munro, J. W. Blunt, P. E. Mulligan, Y. R. Beasley, A. M. Dahlem, W. W. Carmichael, J. Am. Chem. Soc. 1988, 110, 8557. h) E. D. de Silva, D. E. Williams, R. J. Anderson, H. Klix, C. F. B. Holmes, T. M. Allen, Tetrahedron Lett. 1992, 33, 1561.

2 a) Y. Shimohigashi, N. Izumiya, J. Synth. Org. Chem., Jpn. 1978, 36, 1023. b) C. Shin, J. Synth. Org. Chem., Jpn. 1979, 37, 830. c) G. H. Fisher, P. Berryer, J. W. Ryan, V. Chauhan, C. H. Stammer, Arch. Biochem. Biophys. 1981, 211, 269. d) K. Nunami, M. Suzuki, N. Yoneda, Chem. Pharm. Bull. 1982, 30, 4015. e) T. Wakamiya, K. Shimbo, A. Sano, K. Fukase, H. Yasuda, T. Shiba, Pept. Chem. 1982, 1983, 149. f) Y. Shimohigashi, C. H. Stammer, J. Chem. Soc., Perkin Trans. 1 1983, 803. g) R. Jacquier, J. Verducci, Tetrahedron Lett. 1984, 25, 2775. h) S. F. Brady, D. W. Cochran, R. F. Nutt, F. W. Holly, C. D. Bernnett, W. J. Paleveda, P. E. Curley, B. H. Arison, R. Saperstein, D. F. Veber, Int. Pept. Protein Res. 1984, 23, 212.

3 a) I. Photaki, J. Am. Chem. Soc. 1963, 85, 1123. b) D. H. Rich, J. Tam, J. Org. Chem. 1977, 42, 3815. c) D. H. Rich, J. Tam, P. Mathiaparanam, J. A. Grant, C. Mabuni, J. Chem. Soc., Chem. Commun. 1974, 897. d) C. Shin, Y. Yonezawa, K. Unoki, J. Yoshimura, Tetrahedron Lett. 1979, 20, 1049. e) S. Nomoto, A. Sano, T. Shiba, Tetrahedron Lett. 1979, 20, 521. f) P. A. Manis, M. W. Rathke, J. Org. Chem. 1980, 45, 4952. g) U. Schmidt, A. Lieberknecht, J. Wild, Synthesis 1984, 53. h) P. M. T. Ferreira, L. S. Monteiro, G. Pereira, L. Ribeiro, J. Sacramento, L. Silva, Eur. J. Org. Chem. 2007, 5934. i) R. Ramesh, K. De, S. Chandrasekaran, Tetrahedron 2007, 63, 10534.

4 T. Nagano, H. Kinoshita, Bull. Chem. Soc. Jpn. 2000, 73, 1605 .

5 R. Kimura, T. Nagano, H. Kinoshita, Bull. Chem. Soc. Jpn. 2002, 75, 2517.

6 a) A. Srinivasan, R. W. Stephenson, R. K. Olsen, J. Org. Chem. 1977, 42, 2253. b) L. Somekh, A. Shanzer, J. Org. Chem. 1983, 48, 907. c) M. M. Stohlmeyer, H. Tanaka, T. J. Wandless, J. Am. Chem. Soc. 1999, 121, 6100. d) N. O. Silva, A. S. Abreu, P. M. T. Ferreira, L. S. Monteiro, M.-J. R. P. Queiroz, Eur. J. Org. Chem. 2002, 2524. e) F. Yokokawa, T. Shioiri, Tetrahedron Lett. 2002, 43, 8679. f) H. Sai, T. Ogiku, H. Ohmizu, Synthesis 2003, 201.

7 R. Jumnah, J. M. J. Williams, A. C. Williams, Tetrahedron Lett. 1993, 34, 6619.

8 a) Y. Yonezawa, T. Obara, C. Shin, Nippon Kagaku Kaishi 1987, 838. b) H. Poisel, Chem. Ber. 1977, 110, 942; 1977, 110, 948. c) H.-J. Kreuzfeld, C. Döbler, H. W. Krause, C. Facklam, Tetrahedron: Asymmetry 1993, 4, 2047.

9 a) O. Miyata, H. Asai, T. Naito, Chem. Pharm. Bull. 2005, 53, 355. b) D. Crich, A. Banerjee, J. Org. Chem. 2006, 71, 7106. 
10 T. Kawabata, H. Suzuki, Y. Nagae, K. Fuji, Angew. Chem., Int. Ed. 2000, 39, 2155.

11 a) A. Shanzer, L. Somekh, D. Butina, J. Org. Chem. 1979, 44, 3967. b) U. Schmidt, W. Siegel, Tetrahedron Lett. 1987, 28, 2849.

12 Y. Shiraishi, H. Yamauchi, T. Takamura, H. Kinoshita, Bull. Chem. Soc. Jpn. 2004, 77, 2219.

13 P. A. Alexander, S. P. Marsden, D. M. M. Subtil, J. C. Reader, Org. Lett. 2005, 7, 5433.

14 H.-J. Kreuzfeld, U. Schmidt, C. Döbler, H. W. Krause, Tetrahedron: Asymmetry 1996, 7, 1011.

15 E. Tullberg, D. Peters, T. Frejd, J. Organomet. Chem. 2004,
689, 3778.

16 L.-X. Liao, W.-S. Zhou, Tetrahedron 1998, 54, 12571.

17 D. L. Preuss, M. Kellett, J. Antibiot. 1983, 36, 213.

18 J. M. Jiménez, J. Rifé, R. M. Ortuño, Tetrahedron: Asymmetry 1995, 6, 1849.

19 U. Schmidt, R. Meyer, V. Leitenberger, F. Stäbler, A. Lieberknecht, Synthesis 1991, 409.

20 C. Shin, Y. Yonezawa, M. Ikeda, Bull. Chem. Soc. Jpn. 1986, 59, 3573.

21 a) X. Fang, U. K. Bandarage, T. Wang, J. D. Schroeder, D. S. Garvey, J. Org. Chem. 2001, 66, 4019. b) M. F. Semmelhack, Y. Jiang, D. Ho, Org. Lett. 2001, 3, 2403. 